\title{
Dehn twists in Heegaard Floer homology
}

\author{
BIJAN SAHAMIE
}

\begin{abstract}
We derive a new exact sequence in the hat-version of Heegaard Floer homology. As a consequence we see a functorial connection between the invariant of Legendrian knots $\widehat{\mathcal{L}}$ and the contact element. As an application we derive two vanishing results of the contact element making it possible to easily read off its vanishing out of a surgery presentation in suitable situations.
\end{abstract}

57R17; 53D35, 57R58

\section{Introduction}

Heegaard Floer homology is a Floer-type homology theory developed by P Ozsváth and Z Szabó. There are two invariants in Heegaard Floer homology interesting for contact geometry. First to mention is the contact element, introduced in [24] by Ozsváth and Szabó. This contact element is an isotopy invariant of contact structures and an obstruction to overtwistedness. It is useful in the sense that there are examples of contact structures (see Stipsicz $[17 ; 16 ; 18]$ ) where conventional techniques fail to detect, but the contact element is able to detect, tightness versus overtwistedness. The second invariant to mention is the isotopy invariant $\widehat{\mathcal{L}}$ of Legendrian knots found by Lisca, Ozsváth, Stipsicz and Szabó. There is also an isotopy invariant of contact manifolds with boundary (see Honda, Kazez and Matić [12]) in sutured Floer homology (see Juhasz [14]). As a variant of this contact geometric invariant, Honda, Kazez and Matić found and isotopy invariant of Legendrian knots, called $E H$, in the sutured Floer theory. This invariant is related to $\widehat{\mathcal{L}}$ as shown by Stipsicz and Vertesi in [26].

In this paper we start with the observation that the hat-version of knot Floer homology can be defined, and is well-defined, even for knots that are not null-homologous (see Section 2.2.1). Furthermore, we see that in case of the hat-version of the knot Floer homology we can relax the admissibility condition (see Section 2.2.1). After that, in Section 4, we will derive in what way the hat-version of Heegaard Floer homology behaves on Dehn twist changes of the gluing map (see Propositions 4.4 and 4.7). The representation found naturally imposes the existence of an exact sequence (see Corollaries 4.5 and 4.8). In Section 5 we set up invariance properties (see Propositions 5.1 to 5.6) suitable for showing that the maps involved in the sequence are topological, that 
is, they only depend on the cobordism that can be associated to the Dehn twist (see Theorem 5.7). One of the maps involved in the sequence preserves contact geometric information when representing a $(+1)$-contact surgery (see Theorem 6.1). This leads to a functorial connection between the invariant $\widehat{\mathcal{L}}$ and the contact element when performing (+1)-contact surgeries (see Section 6). Finally, in Section 7 we give some applications. The first to mention is Proposition 7.1 in which we give a new proof for a result that can already be derived using known results (see Section 7). A second application is Proposition 7.3 where we give a calculation of a contact element after performing a $(+1)$-contact surgery by using the invariant of Legendrian knots with Theorem 6.1. Finally, with Theorem 7.4, we derive a new vanishing result of the contact element which can be easily read off from a surgery representation of the underlying contact manifold. Everything here is done with $\mathbb{Z}_{2}$-coefficients. A suitable introduction of coherent orientations will be given in a future article.

Acknowledgements The present article contains the results, given in chapter 3 of the author's PhD thesis [25]. The author wants to thank his advisor Hansjörg Geiges for his constructive comments which helped make the exposition clearer at numerous spots. The author wishes to warmly thank András Stipsicz for his help and his constructive criticism on the first version of this article.

\section{Introduction to Heegaard Floer theory}

\subsection{Handle decompositions and Heegaard diagrams}

We briefly review the connection between Heegaard diagrams and handle decompositions to fix our point of view on the subject.

Let $Y$ be a closed, oriented 3-manifold. $Y$ admits a handle decomposition with one 0 -handle $h^{0}$ and one 3 -handle $h^{3}$, a number $l$ of 1 -handles and a number $g$ of 2-handles. The union

$$
H_{0}:=h^{0} \cup_{\partial} h_{0,1}^{1} \cup_{\partial} \ldots \cup_{\partial} h_{0, l}^{1}
$$

is a handlebody of genus $l$. By dualizing the 2-handles and 3-handles in the handle decomposition of $Y$ we see that the union of these is a handlebody $H_{1}$ of genus $g$. Since $Y=H_{0} \cup_{\partial} H_{1}$ is closed obviously the genera of $H_{0}$ and $H_{1}$ coincide. The manifold $Y$ is determined by the following data: The images of the attaching circles of the 2-handles on $\Sigma:=\partial H_{0}$. We can equivalently interpret this handle decomposition as a decomposition relative to the splitting surface $\Sigma$. By dualizing the handlebody $H_{0}$ we can write the manifold $Y$ as

$$
\left(h_{0}^{3} \cup_{\partial} h_{0,1}^{2} \cup_{\partial} \ldots \cup_{\partial} h_{0, g}^{2}\right) \cup_{\partial}(\Sigma \times[0,1]) \cup_{\partial}\left(h_{1,1}^{2} \cup_{\partial} \ldots \cup_{\partial} h_{1, g}^{2} \cup_{\partial} h_{1}^{3}\right) .
$$


The information necessary to describe the 3-manifold $Y$ in terms of a handle decomposition like (2-1) is a triple $(\Sigma, \alpha, \beta)$, where $\Sigma$ is the splitting surface used in the decomposition (2-1), $\alpha=\left\{\alpha_{1}, \ldots, \alpha_{g}\right\}$ are the images of the attaching circles of the $h_{0, i}^{2}$ in $\Sigma \times\{0\}$ and $\beta=\left\{\beta_{1}, \ldots, \beta_{g}\right\}$ the images of the attaching circles of the 2 -handles $h_{1, i}^{2}$ in $\Sigma \times\{1\}$. Observe that the $\alpha$-curves are the co-cores of the 1 -handles in the dual picture, and that sliding the 1 -handle $h_{0, i}^{1}$ over $h_{0, j}^{1}$ means, in the dual picture, that $h_{0, j}^{2}$ is slid over $h_{0, i}^{2}$.

\subsection{Heegaard Floer homologies}

The Heegaard Floer homology groups $\operatorname{HF}^{-}(Y)$ and $\widehat{\mathrm{HF}}(Y)$ of a 3-manifold $Y$ were introduced by Ozsváth and Szabó in [23]. The definition was extended for the case where $Y$ is equipped with a null-homologous knot $K \subset Y$ to variants $\operatorname{HFK}^{-}(Y, K)$, $\widehat{\mathrm{HFK}}(Y, K)$ in Ozsváth-Szabó [21].

A 3-manifold $Y$ can be described by a Heegaard diagram, which is a triple $(\Sigma, \alpha, \beta)$, where $\Sigma$ is an oriented genus-g surface and $\alpha=\left\{\alpha_{1}, \ldots, \alpha_{g}\right\}, \beta=\left\{\beta_{1}, \ldots, \beta_{g}\right\}$ are two sets of pairwise disjoint simple closed curves in $\Sigma$ called attaching circles (see Section 2.2). Each set of curves $\alpha$ and $\beta$ is required to consist of linearly independent curves in $H_{1}(\Sigma, \mathbb{Z})$. In the following we will talk about the curves in the set $\alpha$ (resp. $\beta$ ) as $\alpha$-curves (resp. $\beta$-curves). Without loss of generality we may assume that the $\alpha$-curves and $\beta$-curves intersect transversely. To a Heegaard diagram we may associate the triple $\left(\operatorname{Sym}^{g}(\Sigma), \mathbb{T}_{\alpha}, \mathbb{T}_{\beta}\right)$ consisting of the $g$-fold symmetric power of $\Sigma$,

$$
\operatorname{Sym}^{g}(\Sigma)=\Sigma^{\times g} / S_{g},
$$

and the submanifolds $\mathbb{T}_{\alpha}=\alpha_{1} \times \cdots \times \alpha_{g}$ and $\mathbb{T}_{\beta}=\beta_{1} \times \cdots \times \beta_{g}$. We define $\mathrm{CF}^{-}(\Sigma, \alpha, \beta)$ as the free $\mathbb{Z}_{2}[U]$-module generated by the set $\mathbb{T}_{\alpha} \cap \mathbb{T}_{\beta}$. In the following we will just write $\mathrm{CF}^{-}$. For two intersection points $x, y \in \mathbb{T}_{\alpha} \cap \mathbb{T}_{\beta}$ define $\pi_{2}(x, y)$ to be the set of homology classes of Whitney discs $\phi: \mathrm{D} \longrightarrow \operatorname{Sym}^{g}(\Sigma)(\mathrm{D} \subset \mathbb{C})$ that connect $x$ with $y$. The map $\phi$ is called Whitney if $\phi(D \cap\{R e<0\}) \subset \mathbb{T}_{\alpha}$ and $\phi(\mathrm{D} \cap\{R e>0\}) \subset \mathbb{T}_{\beta}$. We call $\mathrm{D} \cap\{R e<0\}$ the $\alpha$-boundary of $\phi$ and $\mathrm{D} \cap\{R e>0\}$ the $\beta$-boundary of $\phi$. Such a Whitney disc connects $x$ with $y$ if $\phi(i)=x$ and $\phi(-i)=y$. Note that $\pi_{2}(x, y)$ can be interpreted as the subgroup of elements in $H_{2}\left(\operatorname{Sym}^{g}(\Sigma), \mathbb{T}_{\alpha} \cup \mathbb{T}_{\beta}\right)$ represented by discs with appropriate boundary conditions. We endow $\operatorname{Sym}^{g}(\Sigma)$ with a symplectic structure $\omega$. By choosing an almost complex structure $J$ on $\operatorname{Sym}^{g}(\Sigma)$ suitably (see [23]) all moduli spaces of holomorphic Whitney discs are Gromov-compact manifolds. Denote by $\mathcal{M}_{\phi}$ the set of holomorphic Whitney discs in the equivalence class $\phi$, and $\mu(\phi)$ the formal dimension of $\mathcal{M}_{\phi}$. Denote by $\widehat{\mathcal{M}}_{\phi}=\mathcal{M}_{\phi} / \mathbb{R}$ the quotient under the translation action of $\mathbb{R}$ (see [23]). 
Define $H(x, y, k)$ to be the subset of classes in $\pi_{2}(x, y)$ that admit moduli spaces of dimension $k$. Fix a point $z \in \Sigma \backslash(\alpha \cup \beta)$ and define the map

$$
n_{z}: \pi_{2}(x, y) \longrightarrow \mathbb{Z}, \phi \longmapsto \#\left(\phi,\{z\} \times \operatorname{Sym}^{g-1}(\Sigma)\right) .
$$

A boundary operator $\partial^{-}: \mathrm{CF}^{-} \longrightarrow \mathrm{CF}^{-}$is given by defining it on the generators $x$ of $\mathrm{CF}^{-}$by

$$
\partial^{-} x=\sum_{y \in \mathbb{T}_{\alpha} \cap \mathbb{T}_{\beta}} \sum_{\phi \in H(x, y, 1)} \# \widehat{\mathcal{M}}_{\phi} \cdot U^{n_{z}(\phi)} y .
$$

Define $\widehat{\mathrm{CF}}$ to be the free $\mathbb{Z}_{2}-$ module generated by $\mathbb{T}_{\alpha} \cap \mathbb{T}_{\beta}$. By sending $U$ to zero we can define a projection $\pi: \mathrm{CF}^{-} \longrightarrow \widehat{\mathrm{CF}}$. With this projection the differential $\partial^{-}$ induces a morphism $\widehat{\partial}$ on $\widehat{\mathrm{CF}}$. The almost-complex structure $J$ on $\operatorname{Sym}^{g}(\Sigma)$ is chosen in such a way that $\{z\} \times \operatorname{Sym}^{g-1}(\Sigma)$ is a complex submanifold of $\operatorname{Sym}^{g}(\Sigma)$. This means a holomorphic Whitney disc intersects $\{z\} \times \operatorname{Sym}^{g-1}(\Sigma)$ always positively. Thus $\widehat{\partial}$ is a differential on $\widehat{\mathrm{CF}}$. We define

$$
\operatorname{HF}^{-}(Y):=H_{*}\left(\mathrm{CF}^{-}, \partial^{-}\right) \quad \text { and } \widehat{\mathrm{HF}}(Y):=H_{*}(\widehat{\mathrm{CF}}, \widehat{\partial}) \text {. }
$$

These homology groups are topological invariants of the manifold $Y$. We would like to note that not all Heegaard diagrams are suitable for defining Heegaard Floer homology; there is an additional condition that has to be imposed called admissibility. This is a technical condition in the compactification of the moduli spaces of holomorphic Whitney discs. A detailed knowledge of this condition is not important in the remainder of the present article since all constructions are done nicely so that there will never be a problem. We advise the interested reader to consult Ozsváth and Szabó [23].

2.2.1 Knot Floer homology Knot Floer homology is a variant of the Heegaard Floer homology of a manifold. We briefly introduce the theory here and finally argue why the construction carries over verbatim to give an invariant even for knots that are not necessarily null-homologous. For a more detailed treatment we point the reader to the author's PhD thesis [25].

Given a knot $K \subset Y$, we can specify a certain subclass of Heegaard diagrams.

Definition 2.1 A Heegaard diagram $(\Sigma, \alpha, \beta)$ is said to be subordinate to the knot $K$ if $K$ is isotopic to a knot lying in $\Sigma$ and $K$ intersects $\beta_{1}$ once transversely and is disjoint from the other $\beta$-circles.

Since $K$ intersects $\beta_{1}$ once and is disjoint from the other $\beta$-curves we know that $K$ intersects the core disc of the 2 -handle represented by $\beta_{1}$ once and is disjoint from the others (after possibly isotoping the knot $K$ ). 
Lemma 2.2 Every pair $(Y, K)$ admits a Heegaard diagram subordinate to $K$.

Proof By surgery theory (see Gompf and Stipsicz [11, page 104]) we know that there is a handle decomposition of $Y \backslash v K$, that is,

$$
Y \backslash v K \cong\left(T^{2} \times[0,1]\right) \cup_{\partial} h_{2}^{1} \cup_{\partial} \ldots h_{g}^{1} \cup_{\partial} h_{1}^{2} \cup_{\partial} \ldots \cup_{\partial} h_{g}^{2} \cup_{\partial} h^{3}
$$

We close up the boundary $T^{2} \times\{0\}$ with an additional 2-handle $h_{1}^{2 *}$ and a 3 -handle $h^{3}$ to obtain

$$
Y \cong h^{3} \cup_{\partial} h_{1}^{2 *} \cup_{\partial}\left(T^{2} \times I\right) \cup_{\partial} h_{2}^{1} \cup_{\partial} \ldots h_{g}^{1} \cup_{\partial} h_{1}^{2} \cup_{\partial} \ldots \cup_{\partial} h_{g}^{2} \cup_{\partial} h^{3} .
$$

We may interpret $h^{3} \cup_{\partial} h_{1}^{2 *} \cup_{\partial}\left(T^{2} \times[0,1]\right)$ as a 0 -handle $h^{0}$ and a 1 -handle $h_{1}^{1 *}$. Hence, we obtain the following decomposition of $Y$ :

$$
h^{0} \cup_{\partial} h_{1}^{1 *} \cup_{\partial} h_{2}^{1} \cup_{\partial} \ldots \cup_{\partial} h_{g}^{1} \cup_{\partial} h_{1}^{2} \cup_{\partial} \ldots \cup_{\partial} h_{g}^{2} \cup_{\partial} h^{3} .
$$

We get a Heegaard diagram $(\Sigma, \alpha, \beta)$ where $\alpha=\left\{\alpha_{1}\right\}^{*} \cup\left\{\alpha_{2}, \ldots, \alpha_{g}\right\}$ are the co-cores of the 1 -handles and $\beta=\left\{\beta_{1}, \ldots, \beta_{g}\right\}$ are the attaching circles of the 2 -handles.

Having fixed such a Heegaard diagram $(\Sigma, \alpha, \beta)$ we can encode the knot $K$ in a pair of points. After isotoping $K$ onto $\Sigma$, we fix a small interval $I$ in $K$ containing the intersection point $K \cap \beta_{1}$. This interval should be chosen small enough such that $I$ does not contain any other intersections of $K$ with other attaching curves. The boundary $\partial I$ of $I$ determines two points in $\Sigma$ that lie in the complement of the attaching circles, that is, $\partial I=z-w$, where the orientation of $I$ is given by the knot orientation. This leads to a doubly-pointed Heegaard diagram $(\Sigma, \alpha, \beta, w, z)$. Conversely, a doubly-pointed Heegaard diagram uniquely determines a topological knot class: Connect $z$ with $w$ in the complement of the attaching circles $\alpha$ and $\beta \backslash \beta_{1}$ with an arc $\delta$ that crosses $\beta_{1}$ once. Connect $w$ with $z$ in the complement of $\beta$ using an arc $\gamma$. The union $\delta \cup \gamma$ represents the knot class of $K$. The orientation on $K$ is given by orienting $\delta$ such that $\partial \delta=z-w$. If we use a different path $\tilde{\gamma}$ in the complement of $\beta$, we observe that $\tilde{\gamma}$ is isotopic to $\gamma$ (in $Y$ ): Since $\Sigma \backslash \beta$ is a sphere with holes an isotopy can move $\gamma$ across the holes by doing handle slides. Isotope the knot along the core discs of the $2-$ handles to cross the holes of the sphere. Indeed, the knot class does not depend on the specific choice of $\delta$-curve.

The knot chain complex $\widehat{\mathrm{CFK}}(Y, K)$ is the free $\mathbb{Z}_{2}$-module generated by the intersections $\mathbb{T}_{\alpha} \cap \mathbb{T}_{\beta}$. The boundary operator $\hat{\partial}^{w}$, for $x \in \mathbb{T}_{\alpha} \cap \mathbb{T}_{\beta}$, is defined by

$$
\widehat{\partial}^{w}(x)=\sum_{y \in \mathbb{T}_{\alpha} \cap \mathbb{T}_{\beta}} \sum_{\phi \in H(x, y, 1)} \# \widehat{\mathcal{M}}_{\phi} \cdot y,
$$


where $H(x, y, 1) \subset \pi_{2}(x, y)$ are the homotopy classes with $\mu=1$ and $n_{z}=n_{w}=0$. We denote by $\widehat{\operatorname{HFK}}(Y, K)$ the associated homology theory $H_{*}\left(\widehat{\mathrm{CFK}}(Y, K), \widehat{\partial}^{w}\right)$. The crucial observation for showing invariance is, that two Heegaard diagrams subordinate to a given knot can be connected by moves that respect the knot complement.

Lemma 2.3 (Ozsváth and Szabó [21]) Let $(\Sigma, \alpha, \beta, z, w)$ and $\left(\Sigma^{\prime}, \alpha^{\prime}, \beta^{\prime}, z^{\prime}, w^{\prime}\right)$ be two Heegaard diagrams subordinate to a given knot $K \subset Y$. Let $I$ denote the interval inside $K$ connecting $z$ with $w$, interpreted as sitting in $\Sigma$. Then these two diagrams are isomorphic after a sequence of the following moves:

$\left(m_{1}\right)$ Handle slides and isotopies among the $\alpha$-curves. These isotopies may not cross $I$.

$\left(m_{2}\right)$ Handle slides and isotopies among the $\beta_{2}, \ldots, \beta_{g}$. These isotopies may not cross $I$.

( $\left.m_{3}\right)$ Handle slides of $\beta_{1}$ over the $\beta_{2}, \ldots, \beta_{g}$ and isotopies.

$\left(m_{4}\right)$ Stabilizations/destabilizations.

For the convenience of the reader we include a short proof of this lemma.

Proof By [11, Theorem 4.2.12] we can transform two relative handle decompositions into each other by isotopies, handle slides and handle creation/annihilation of the handles written at the right of $T^{2} \times[0,1]$ in (2-2). Observe that the 1 -handles may be isotoped along the boundary $T^{2} \times\{1\}$. Thus, we can transform two Heegaard diagrams into each other by handle slides, isotopies, creation/annihilation of the 2-handles $h_{i}^{2}$ and we may slide the $h_{i}^{1}$ over $h_{j}^{1}$ and $h_{1}^{1 *}$ (the latter corresponds to $h_{i}^{1}$ sliding over the boundary $T^{2} \times\{1\} \subset T^{2} \times I$ by an isotopy). But we are not allowed to move $h_{1}^{1 *}$ off the 0 -handle. In this case we would lose the relative handle decomposition. In terms of Heegaard diagrams we see that these moves exactly translate into the moves given in $\left(m_{1}\right)$ to $\left(m_{4}\right)$. Just note that sliding the $h_{i}^{1}$ over $h_{1}^{1 *}$, in the dual picture, looks like sliding $h_{1}^{2 *}$ over the $h_{i}^{2}$. This corresponds to move $\left(m_{3}\right)$.

Proposition 2.4 (Sahamie [25, Proposition 2.4.4]) Let $K \subset Y$ be an arbitrary knot. The knot Floer homology group $\widehat{\operatorname{HFK}}(Y, K)$ is a topological invariant of the knot type of $K$ in $Y$. These homology groups split with respect to $\operatorname{Spin}^{c}(Y)$.

There are no homological requirements on the knot $K$ needed for proving Lemma 2.2 and Lemma 2.3. Thus we may define the knot Floer homology for an arbitrarily chosen pair $(Y, K)$. To conclude that the defined groups are indeed invariants of the pair $(Y, K)$ we have to observe that every move, $\left(m_{1}\right)$ to $\left(m_{4}\right)$, induces an isomorphism between 
the respective knot Floer homologies. The invariance proof of knot Floer homology Ozsváth and Szabó give in [21] uses the maps from the invariance proof of Heegaard Floer homology with just one slight modification. In knot Floer homology they require the holomorphic discs counted to have trivial intersection number $n_{w}$. The positivity of intersections in the holomorphic case and the additivity of the intersection number imply that the associated maps between the knot Floer homologies are isomorphisms. We do not need any homological information of the knot $K$. For details we point the reader to the author's $\mathrm{PhD}$ thesis [25].

2.2.2 Admissibility of $\widehat{\mathrm{HFK}}$ Finally, we would like to address the admissibility conditions imposed on the Heegaard diagrams, used in the definition of the knot Floer homologies. We may relax the admissibility condition, given by Ozsvaáth and Szabó (see [21]) and still get well-defined knot invariants. A periodic domain $\mathcal{D}$ is a linear combination of the components $\Sigma \backslash\{\alpha \cup \beta\}$ such that the boundary of $\mathcal{D}$ consists of a linear combination of $\alpha$-curves and $\beta$-curves and such that $n_{z}(\mathcal{D})=0$, where $n_{z}(\mathcal{D})$ is the multiplicity of $\mathcal{D}$ at the region containing the base point $z$. Furthermore, we denote by $\mathcal{H}(\mathcal{D})$ its associated homology class which is given by closing the boundary components of $\mathcal{D}$ with the cores of the 2 -handles associated to the $\alpha$-curves and $\beta$-curves.

Definition 2.5 We call a doubly-pointed Heegaard diagram $(\Sigma, \alpha, \beta, w, z)$ extremely weakly admissible for the $\operatorname{Spin}^{c}$-structure $s$ if for every non-trivial periodic domain, with $n_{w}=0$ and $\left\langle c_{1}(s), \mathcal{H}(\mathcal{D})\right\rangle=0$, the domain has both positive and negative coefficients.

It is not hard to see that the following result holds.

Theorem 2.6 (Sahamie [25, Theorem 2.4.6]) Let $(\Sigma, \alpha, \beta, w, z)$ be an extremely weakly admissible Heegaard diagram, then $\hat{\partial}^{w}$ is well-defined and a differential.

\subsection{Contact structures}

A 3-dimensional contact manifold is a pair $(Y, \xi)$ where $Y$ is a 3-dimensional manifold and $\xi \subset T Y$ a hyperplane bundle, that can be written as the kernel of a 1-form $\alpha$ with the property

$$
\alpha \wedge d \alpha \neq 0 \text {. }
$$

1 -forms with the property (2-3) are called contact forms. The contact form $\alpha$ is not uniquely determined. The existence of a contact form implies that $T Y / \xi$ is a 1dimensional trivial bundle. Thus there are non-vanishing vector fields on $Y$ transverse 
to $\xi$. The vector field $R_{\alpha}$ defined by the conditions

$$
\alpha\left(R_{\alpha}\right) \neq 0 \text { and } \iota_{R_{\alpha}} d \alpha=0
$$

is called Reeb field of the contact form $\alpha$. Two contact manifolds $(Y, \xi)$ and $\left(Y^{\prime}, \xi^{\prime}\right)$ are called contactomorphic if there is a diffeomorphism $\phi: Y \longrightarrow Y^{\prime}$ such that $T \phi(\xi)=\xi^{\prime}$. A diffeomorphism preserving contact structures in this manner is called contactomorphism. Every contact manifold is locally contactomorphic to the standard contact space $\left(\mathbb{R}^{3}, \xi_{s t d}\right)$, where $\xi_{s t d}$ is the contact structure given by the kernel of the 1 -form $d z-y d x$ (Darboux's theorem). This property tells us that locally contact manifolds cannot be distinguished, and ,thus, invariants of contact manifolds always have to be of global nature. An important property of contact structures is known as Gray stability. Gray stability means that each smooth homotopy of contact structures $\left(\xi_{t}\right)_{t_{\in}[0,1]}$ is induced by an ambient isotopy $\phi_{t}$ of the underlying manifold, that is, such that $T \phi_{t}\left(\xi_{0}\right)=\xi_{t}$. This property naturally gives a connection between contact structures and the topology of the manifold. Submanifolds tangent to the contact structure are also interesting objects to study. The contact condition implies that on a 3-dimensional contact manifold $(Y, \xi)$ only 1-dimensional submanifolds, that is, knots and links, can be tangent to $\xi$. These submanifolds are called Legendrian knots and links. Their investigation is naturally motivated by the contact-analogue of surgery, called contact surgery. Contact surgery in arbitrary dimensions was introduced by Eliashberg in [8]. His construction, in dimension 3 , corresponds to $(-1)$-contact surgeries. For 3dimensional contact manifolds Ding and Geiges gave in [2] a definition of contact $r$-surgeries (see also [3] by the same authors) for arbitrary $r \in \mathbb{Q}>0$. It is nowadays one of the most significant tools for 3-dimensional contact geometry.

\subsection{Open books, the contact element and the invariant LOSS}

2.4.1 Open books and the contact element We start by recalling some facts about open book decompositions of 3-manifolds. For details we point the reader to Etnyre [9].

An open book is a pair $(P, \phi)$ consisting of an oriented genus- $g$ surface $P$ with boundary and a homeomorphism $\phi: P \longrightarrow P$ that is the identity near the boundary of $P$. The surface $P$ is called page and $\phi$ the monodromy. Recall that an open book $(P, \phi)$ gives rise to a 3 -manifold by the following construction: Let $c_{1}, \ldots, c_{k}$ denote the boundary components of $P$. Observe that

$$
(P \times[0,1]) /(p, 1) \sim(\phi(p), 0)
$$

is a 3 -manifold with boundary given by the tori

$$
\left(\left(c_{i} \times[0,1]\right) /(p, 1) \sim(p, 0)\right) \cong c_{i} \times \mathbb{S}^{1} .
$$


Fill in each of the holes with a full torus $\mathrm{D}^{2} \times \mathbb{S}^{1}$ : we glue a meridional disc $\mathrm{D}^{2} \times\{\star\}$ onto $\{\star\} \times \mathbb{S}^{1} \subset c_{i} \times \mathbb{S}^{1}$. In this way we define a closed, oriented 3-manifold $Y(P, \phi)$. Denote by $B$ the union of the cores of the tori $\mathrm{D}^{2} \times \mathbb{S}^{1}$. The set $B$ is called binding. Observe that the definition of $Y(P, \phi)$ defines a fibration

$$
P \hookrightarrow Y(P, \phi) \backslash B \longrightarrow \mathbb{S}^{1} .
$$

Consequently, an open book gives rise to a Heegaard decomposition of $Y(P, \phi)$ and, thus, induces a Heegaard diagram of $Y(P, \phi)$. To see this we have to identify a splitting surface of $Y(P, \phi)$, that is, a surface $\Sigma$ that splits the manifold into two components. Observe that the boundary of each fiber lies on the binding $B$. Thus gluing together two fibers yields a closed surface $\Sigma$ of genus $2 g$. The surface $\Sigma$ obviously splits $Y(P, \phi)$ into two components and can therefore be used to define a Heegaard decomposition of $Y(P, \phi)$ (see Geiges [10]).

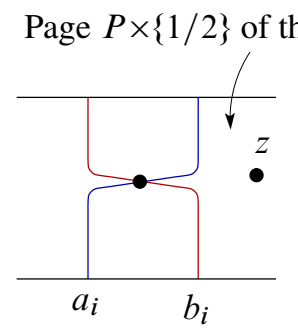

Figure 1: Definition of $b_{i}$ and positioning of the point $z$

Let $a=\left\{a_{1}, \ldots, a_{n}\right\}$ be a cut system of $P$, that is, a set of disjoint properly embedded arcs such that $P \backslash\left\{a_{1}, \ldots, a_{n}\right\}$ is a disc. One can easily show that being a cut system implies that $n=2 g$. Choose the splitting surface

$$
\Sigma:=P \times\{1 / 2\} \cup_{\partial}(-P) \times\{1\}
$$

and let $\bar{a}_{i}$ be the curve $a_{i} \subset P \times\{1 / 2\}$ with opposite orientation, interpreted as a curve in $(-P) \times\{0\}$. Then define $\alpha_{i}:=a_{i} \cup \bar{a}_{i}$. The curves $b_{i}$ are isotopic push-offs of the $a_{i}$. We choose them like indicated in Figure 1: We push the $b_{i}$ off the $a_{i}$ by following with $\partial b_{i}$ the positive boundary orientation of $\partial P$. Finally set $\beta_{i}:=b_{i} \cup \overline{\phi\left(b_{i}\right)}$. The data $(\Sigma, \alpha, \beta)$ define a Heegaard diagram of $Y(P, \phi)$ (see Honda, Kazez and Matić [13]). There is one intersection point of $\mathbb{T}_{\alpha} \cap \mathbb{T}_{\beta}$ sitting on $P \times\{1 / 2\}$. Denote this point by $E H(P, \phi, a)$.

There is a natural way to define a cohomology theory from a given homology (see Bredon [1]): Use the Hom-functor to define a cochain-module and use the naturally induced boundary to give the module the structure of a chain complex. We can define the 
Heegaard Floer cohomology of a manifold $Y$ the same way. One can easily show that the Heegaard Floer cohomology of a manifold $Y$ is isomorphic to the Heegaard Floer homology of $-Y$ (see [23]). Observe that if $(\Sigma, \alpha, \beta)$ is a Heegaard diagram for $Y$ then $(-\Sigma, \alpha, \beta)$ is a Heegaard diagram for $-Y$. The change of the surface orientation affects the boundary operator through a modification of the boundary conditions of the Whitney discs: we count holomorphic discs $\phi$ with $\phi(i)=x, \phi(-i)=y$ and $\phi\left(\mathrm{D}^{2} \cap \operatorname{Re}<0\right) \subset \mathbb{T}_{\beta}$ and $\phi\left(\mathrm{D}^{2} \cap \operatorname{Re}<0\right) \subset \mathbb{T}_{\alpha}$ (note that we switched the boundary conditions). Hence the Heegaard Floer cohomology of $Y$ is given by the data $(\Sigma, \beta, \alpha)$ (we changed the position of $\alpha$ and $\beta$ ). The point $E H(P, \phi, a)$ can be interpreted as a generator of $\widehat{\mathrm{CF}}(-Y)$. In this case $E H(P, \phi, a)$ is indeed a cycle and thus defines a cohomology class $c(P, \phi) \in \widehat{\mathrm{HF}}(-Y)$. The class $[E H(P, \phi, a)]$ does not depend on the choice of cut system $a$.

Recall the connection between open books and contact structures on 3-manifolds (see Etnyre [9]). Every contact structure gives rise to an adapted open book decomposition. The open book is uniquely determined up to positive Giroux stabilizations. Given a contact structure $\xi$ on a manifold $Y$ we may define $c(Y, \xi):=c(P, \phi)$, where $(P, \phi)$ is an open book decomposition of $Y$ adapted to the contact structure $\xi$. The class $c(P, \phi)$ is invariant under handle slides, isotopies and positive Giroux stabilizations (see Honda, Kazez and Matić [13]). Thus $c(P, \phi)$ does not depend on the specific choice of adapted open book and is an isotopy invariant of the contact manifold $(Y, \xi)$. This cohomology class is called contact element.

2.4.2 The invariant LOSS Ideas very similar to those used to define the contact element are can be utilized to define an invariant of Legendrian knots we will briefly call LOSS. This invariant is due to Lisca, Ozsváth, Stipsicz and Szabó and was defined in [15]. It is basically the contact element, but now it is interpreted as sitting in a filtered Heegaard Floer complex. The filtration is constructed with respect to a fixed Legendrian knot.

Page $P \times\{1 / 2\}$ of the open book

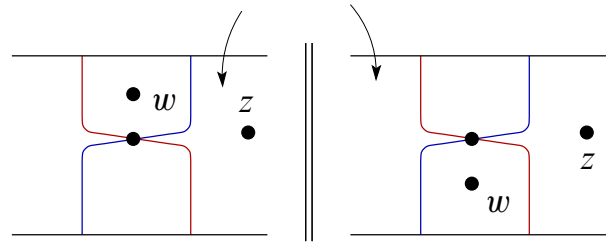

Figure 2: Positioning of the point $w$ depending on the knot orientation 
Let $(Y, \xi)$ be a contact manifold and $L \subset Y$ a Legendrian knot. There is an open book decomposition of $Y$ subordinate to $\xi$ such that $L$ sits on the page $P \times\{1 / 2\}$ of the open book. Choose a cut system that induces an $L$-adapted Heegaard diagram (see Section 4.1, Definition 4.1 and Lemma 4.2). Figure 2 illustrates the positioning of a point $w$ in the Heegaard diagram induced by the open book. Similar to the case of the contact element there is one specific generator of $\widehat{\mathrm{CF}}(-Y)$ sitting of $P \times\{1 / 2\}$. This element may be interpreted as sitting in $\widehat{\mathrm{CFK}}(-Y, L)$ and is a cycle there, too. The induced element in the knot Floer homology is denoted by $\widehat{\mathcal{L}}(L)$.

Remark 1 Since this is an important issue we would like to recall the relation between the pair $(w, z)$ and the knot orientation. In homology we connect $z$ with $w$ in the complement of the $\alpha$-curves and $w$ with $z$ in the complement of the $\beta$-curves (oriented as is obvious from definition). In cohomology we orient in the opposite manner, that is, we move from $z$ to $w$ in the complement of the $\beta$-curves and from $w$ to $z$ in the complement of the $\alpha$-curves.

\section{Algebraic preliminaries}

We outline some algebraic tools used in the next sections. We present this material for the sake of completeness.

Lemma 3.1 Suppose we are given two complexes $\left(C, \partial_{C}\right)$ and $\left(D, \partial_{D}\right)$ and a morphism $f: D \longrightarrow C$ of complexes. Then $\left(C \oplus D, \partial^{f}\right)$ is a chain complex where $\partial^{f}:=\partial_{C}+f-\partial_{D}$, that is,

$$
\partial^{f}=\left(\begin{array}{cc}
\partial_{C} & f \\
0 & -\partial_{D}
\end{array}\right)
$$

Proof For $(p, q) \in C \oplus D$ we calculate

$$
\begin{aligned}
\left(\partial^{f}\right)^{2}(p, q) & =\partial^{f}\left(\partial_{C} p+f(q),-\partial_{D} q\right) \\
& =\left(\partial_{C}^{2} p+\partial_{C} f(p)+f\left(-\partial_{D} p\right), \partial_{D}^{2} p\right) \\
& =0,
\end{aligned}
$$

where the last equality holds since $\partial_{C}$ and $\partial_{D}$ are differentials and $f$ is a chain map. $\square$ A nice, immediate consequence of this construction is the following Lemma.

Lemma 3.2 There is a long exact sequence

$$
\ldots \stackrel{-f_{*}}{\longrightarrow} H_{*}\left(C, \partial_{C}\right) \stackrel{\Gamma_{1}}{\longrightarrow} H_{*}\left(C \oplus D, \partial^{f}\right) \stackrel{\Gamma_{2}}{\longrightarrow} H_{*}\left(D,-\partial_{D}\right) \stackrel{-f_{*}}{\longrightarrow} \ldots
$$


where $f_{*}$ is the map in homology induced by $f$, and $\Gamma_{1}$ and $\Gamma_{2}$ are given as follows:

- $\Gamma_{1}$ is induced by the map

$$
\gamma_{1}:\left(C, \partial_{C}\right) \longrightarrow\left(C \oplus D, \partial^{f}\right), x \longmapsto x \oplus 0 ;
$$

- $\Gamma_{2}$ is induced by the map

$$
\gamma_{2}:\left(C \oplus D, \partial^{f}\right) \longrightarrow\left(D,-\partial_{D}\right), x \oplus y \longmapsto-y .
$$

Proof We first have to see that $\gamma_{1}$ and $\gamma_{2}$ are chain maps. Given an element $c \in C$, observe that

$$
\gamma_{1}\left(\partial_{C} c\right)=\partial_{C} c=\partial^{f} c=\partial^{f} \gamma_{1}(c) .
$$

Furthermore, we see that

$$
\gamma_{2}\left(\partial^{f}(c \oplus 0)\right)=\gamma_{2}\left(\partial_{C} c\right)=0=\gamma_{2}(c \oplus 0)=-\partial_{D}\left(\gamma_{2}(c \oplus 0)\right) .
$$

We continue with an element $d \in D$ :

$$
\gamma_{2}\left(\partial^{f}(0 \oplus d)\right)=\gamma_{2}\left(f(d)-\partial_{D}(d)\right)=\partial_{D}(d)=-\partial_{D}\left(\gamma_{2}(0 \oplus d)\right) .
$$

Thus, both $\gamma_{1}$ and $\gamma_{2}$ are chain maps. Finally, $\gamma_{1}$ and $\gamma_{2}$ obviously fit into the short exact sequence

$$
0 \longrightarrow\left(C, \partial_{C}\right) \stackrel{\gamma_{1}}{\longrightarrow}\left(C \oplus D, \partial^{f}\right) \stackrel{\gamma_{2}}{\longrightarrow}\left(D,-\partial_{D}\right) \longrightarrow 0
$$

of chain complexes. Hence, by standard results in algebraic topology (see Bredon [1]) this short exact sequence induces a long exact sequence

$$
\ldots \stackrel{\partial_{*}}{\longrightarrow} H_{*}\left(C, \partial_{C}\right) \stackrel{\Gamma_{1}}{\longrightarrow} H_{*}\left(C \oplus D, \partial^{f}\right) \stackrel{\Gamma_{2}}{\longrightarrow} H_{*}\left(D,-\partial_{D}\right) \stackrel{\partial_{*}}{\longrightarrow} \ldots
$$

It remains to show that the connecting homomorphism $\partial_{*}$ equals $-f_{*}$. Recall that for $d \in \operatorname{ker}\left(\partial_{D}\right)$ the morphism $\partial_{*}$ is defined by

$$
\partial_{*}[d]=\left[\gamma_{1}^{-1}\left(\partial^{f}\left(\gamma_{2}^{-1}(d)\right)\right)\right] .
$$

Of course, $\gamma_{1}$ and $\gamma_{2}$ are not necessarily invertible. However, we take the preimages as given in the equation, and, by standard algebraic topology, all the elements in the preimage will belong to the same equivalence class. Observe:

$$
\begin{aligned}
\partial_{*}[d] & =\left[\gamma_{1}^{-1}\left(\partial^{f}\left(\gamma_{2}^{-1}(d)\right)\right)\right] \\
& =\left[\gamma_{1}^{-1}\left(\partial^{f}(0 \oplus-d)\right)\right] \\
& =\left[\gamma_{1}^{-1}(-f(d))\right] \\
& =-[f(d)] \\
& =-f_{*}[d]
\end{aligned}
$$


Of course, the whole construction works if $f$ goes the other way, that is, $f: C \longrightarrow D$. In this case we form the complex $C \oplus D$ with the differential

$$
\partial_{f}=\left(\begin{array}{cc}
\partial_{C} & 0 \\
f & -\partial_{D}
\end{array}\right) \text {. }
$$

In an analogous manner we obtain a long exact sequence

$$
\ldots \stackrel{-f_{*}}{\longrightarrow} H_{*}\left(D,-\partial_{D}\right) \stackrel{\Gamma_{1}}{\longrightarrow} H_{*}\left(C \oplus D, \partial_{f}\right) \stackrel{\Gamma_{2}}{\longrightarrow} H_{*}\left(C, \partial_{C}\right) \stackrel{-f_{*}}{\longrightarrow} \ldots
$$

\section{Two new exact sequences in Heegaard Floer homology}

\subsection{Positive Dehn twists}

Let an open book $(P, \phi)$ and a homologically essential closed curve $\delta$ in $P$ be given. We first ask how a Dehn twist along $\delta$ would change the associated Heegaard Floer homology. To do that, we first have to see that there is a specific choice of attaching circles (see Section 2.4) that are - in a sense - adapted to the closed curve $\delta$.

Definition 4.1 Let a Heegaard diagram $(\Sigma, \alpha, \beta)$ and a homologically essential, simple, closed curve $\delta$ on $\Sigma$ be given. The Heegaard diagram $(\Sigma, \alpha, \beta)$ is called $\delta$-adapted if the following conditions hold.

(1) It is induced by an open book and the pair $\alpha, \beta$ is induced by a cut system (see Section 2.4) for this open book.

(2) The curve $\delta$ intersects $\beta_{1}$ once and does not intersect any other of the $\beta_{i}, i \geq 2$.

We can always find $\delta$-adapted Heegaard diagrams. This is already stated in HondaKazez-Matić [13] and Lisca-Ozsváth-Stipsicz-Szabó [15] but not proved. We wish to give a proof because this specific choice is crucial throughout this article.

Lemma 4.2 Let $(P, \phi)$ be an open book and $\delta \subset P$ a homologically essential closed curve. There is a choice of cut system on $P$ that induces a $\delta$-adapted Heegaard diagram.

Observe that $a_{1}, \ldots, a_{n}$ to be a cut system of a page $P$ essentially means to be a basis of $H_{1}(P, \partial P)$ : Suppose the curves are not linearly independent. In this case we are able to identify a surface $F \subset P, F \neq P$, bounding a linear combination of some of the curves $a_{i}$. But this means the cut system disconnects the page $P$ in contradiction to the 
definition. Conversely, suppose the curves in the cut system are homologically linearly independent. In this case the curves cannot disconnect the page. If they disconnected, we could identify a surface $F$ in $P$ with boundary a linear combination of some of the $a_{i}$. But this contradicts their linear independence. The fact that $\Sigma \backslash\left\{a_{1}, \ldots, a_{n}\right\}$ is a disc shows that every element in $H_{1}(P, \partial P)$ can be written as a linear combination of the curves $a_{1}, \ldots, a_{n}$.

Proof Without loss of generality, we assume that $P$ has connected boundary: Suppose the boundary of $P$ has two components. Choose a properly embedded arc connecting both components of $\partial P$. Define this curve to be the first curve $a_{0}$ in a cut system. Cutting out this curve $a_{0}$, we obtain a surface with connected boundary. The curve $a_{0}$ determines two segments $S_{1}$ and $S_{2}$ in the connected boundary. We can continue using the construction process for connected binding we state below. We just have to check the boundary points of the curves to remain outside of the segments $S_{1}$ and $S_{2}$. Given that $P$ has more than two boundary components, we can, with this algorithm, inductively decrease the number of boundary components.

The map $\phi$ is an element of the mapping class group of $P$. Thus, if $\left\{a_{1}, \ldots, a_{n}\right\}$ is a cut system, then $\left\{\phi\left(a_{1}\right), \ldots, \phi\left(a_{n}\right)\right\}$ is a cut system, too. It suffices to show that there is a cut system $\left\{a_{1}, \ldots, a_{n}\right\}$ such that $\delta$ intersects $a_{i}$ once if and only if $i=1$.

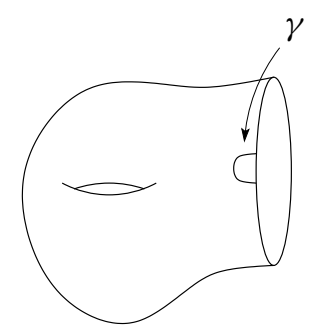

Figure 3: Possible choice of curve $\gamma$

We start by taking a band sum of $\delta$ with a small arc $\gamma$ as shown in Figure 3. We are free to choose the arc $\gamma$. Denote the result of the band sum by $a_{2}$. The arc $a_{2}$ indeed bounds a compressing disc in the respective handlebody because its boundary lies on $\partial P$. Because of our prior observation it suffices to show that $a_{2}$ is a primitive class in $H_{1}(P, \partial P)$. This can be seen with an elementary homological argument using a Mayer-Vietoris computation and the lemma below. Cut open the surface along $\delta$. We obtain two new boundary components, $C_{1}$ and $C_{2}$ say, which we can connect with the boundary of $P$ with two arcs. These two arcs, in $P$, determine a properly embedded curve, $a_{1}$ say, whose boundary lies on $\partial P$. Furthermore, $a_{1}$ intersects $\delta$ in one single 
point, transversely. The curve $a_{1}$ is primitive, too. To see, that we can extend to a cut system such that $\delta$ is disjoint from $a_{3}, \ldots, a_{n}$, cut open the surface $P$ along $\delta$ and $a_{1}$. We obtain a surface $P^{\prime}$ with one boundary component. The curves $\delta$ and $a_{1}$ determine 4 segments, $S_{1}, \ldots, S_{4}$ say, in this boundary. We extend $a_{2}$ to a cut system $a_{2}, \ldots, a_{n}$ of $P^{\prime}$ and arrange the boundary points of the curves $a_{3}, \ldots, a_{n}$ to be disjoint from $S_{1}, \ldots, S_{4}$. The set $a_{1}, \ldots, a_{n}$ is a cut system of $P$ with the desired properties.

As a consequence of the proof we may arrange $\delta$ to be a push-off of $a_{2}$ outside a small neighborhood where the band sum is performed. Geometrically spoken, we cut open $\delta$ at one point, and move the boundaries to $\partial P$ to get $a_{2}$. Given a positive Giroux stabilization, we can find a special cut system which is adapted to the curve $\gamma$. It is not hard to see that there is only one homotopy class of triangles that connect the old with the new contact element and that the associated moduli space is a one-point space.

Lemma 4.3 An embedded circle $\delta$ in an orientable, closed surface $\Sigma$ which is homologically essential is a primitive class of $H_{1}(\Sigma, \mathbb{Z})$.

Proof Cut open the surface $\Sigma$ along $\delta$. We obtain a connected surface $S$ with two boundary components since $\delta$ is homologically essential in $\Sigma$. We can recover the surface $\Sigma$ by connecting both boundary components of $S$ with a 1-handle and then capping off with a disc. There is a knot $K \subset S \cup h^{1}$ intersecting the co-core of $h^{1}$ only once and intersecting $\delta$ only once, too. To construct this knot take a union of two arcs in $S \cup h^{1}$ in the following way: Namely, define $a$ as the core of $h^{1}$, that is, as $D^{1} \times\{0\} \subset D^{1} \times D^{1} \cong h^{1}$ and let $b$ be a curve in $S$, connecting the two components of the attaching sphere $h^{1}$ in $\partial S$. This curve exists since $\delta$ is homologically essential which implies that it is non-separating. We define $K$ to be $a \cup b$. Obviously,

$$
\pm 1=\#(K, \delta)=\langle P D[K],[\delta]\rangle .
$$

Since $H_{1}(\Sigma ; \mathbb{Z})$ is torsion free $H^{1}(\Sigma ; \mathbb{Z}) \cong \operatorname{Hom}\left(H_{1}(\Sigma ; \mathbb{Z}), \mathbb{Z}\right)$. Thus, $[\delta]$ is primitive.

Figure 4 depicts a small neighborhood of the point $\delta \cap \beta_{1}$ in the Heegaard diagram induced by the open book decomposition. The page at the right side of the boundary pictured in Figure 4 is $P \times\{1 / 2\}$. The dotted line indicates the neighborhood of $\partial P$ where the monodromy $\phi$ is the identity. The proof of Lemma 4.2 shows that we can arrange a neighborhood of $\delta \cap \beta_{1}$ to look like in Figure 4, that is, it is possible to arrange the curve $\delta$ and the attaching circles like indicated in Figure 4 due to the arguments given in the proof of Lemma 4.2. 


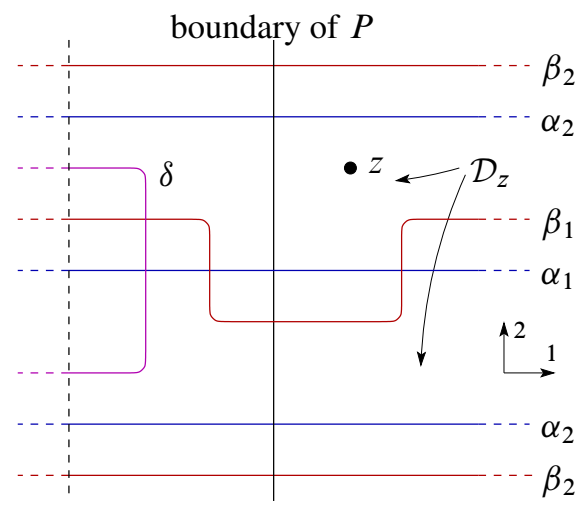

Figure 4: A small neighborhood of $\delta \cap \beta_{1}$ in the Heegaard surface $\Sigma=$ $P \times\{1 / 2\} \cup(-P) \times\{0\}$

With respect to the surface orientation given in Figure 4 this is the appropriate setup for performing a positive Dehn twist along $\delta$ : Denote by $\beta^{\prime}$ the $\beta$-curves after performing the Dehn twist. Obviously, $\beta^{\prime}=\left\{\beta_{1}^{\prime}, \beta_{2}, \ldots \beta_{2 g}\right\}$. Observe that

$$
\mathbb{T}_{\alpha} \cap \mathbb{T}_{\beta^{\prime}}=\mathbb{T}_{\alpha} \cap \mathbb{T}_{\beta} \sqcup \mathbb{T}_{\alpha} \cap \mathbb{T}_{\delta},
$$

where $\mathbb{T}_{\delta}$ is given by the set $\delta=\left\{\delta, \beta_{2}, \ldots, \beta_{2 g}\right\}$ (by abuse of notation since $\delta$ also denotes the curve on $P$ but what is meant will be clear from the context). The set of curves $\delta$ may be interpreted as a set of attaching circles. In the following we will call the arc $\beta_{1}^{\prime} \cap \beta_{1}$ the $\beta$-part of $\beta_{1}^{\prime}$ and the arc $\beta_{1}^{\prime} \cap \delta$ the $\delta$-part of $\beta_{1}^{\prime}$. Figure 5 depicts the situation before and after the Dehn twist.
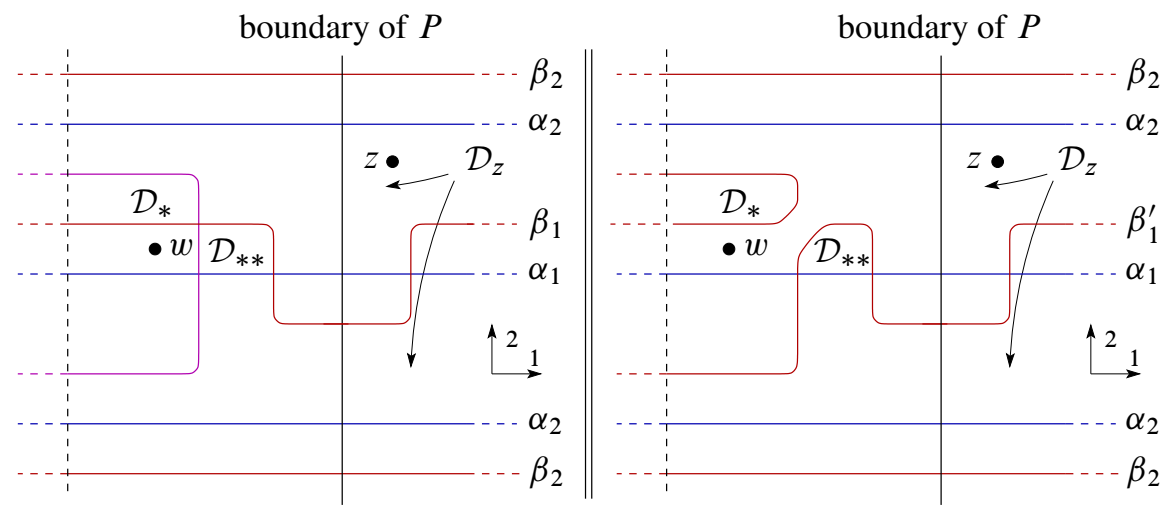

Figure 5: Before and after the positive Dehn twist 
The main observation is that there can be no holomorphic disc in $\left(\Sigma, \alpha, \beta^{\prime}\right)$ that connects a $\mathbb{T}_{\alpha} \cap \mathbb{T}_{\beta}$-intersection of $\mathbb{T}_{\alpha} \cap \mathbb{T}_{\beta^{\prime}}$ with a $\mathbb{T}_{\alpha} \cap \mathbb{T}_{\delta}$-intersection of $\mathbb{T}_{\alpha} \cap \mathbb{T}_{\beta^{\prime}}$. Suppose there is a disc $\phi$ starting at $x \in \mathbb{T}_{\alpha} \cap \mathbb{T}_{\beta}$ and going to $y \in \mathbb{T}_{\alpha} \cap \mathbb{T}_{\delta}$ along its $\alpha$-boundary. Then, at the $\beta$-boundary, the disc $\phi$ has to run from $y$ to $x$ along the $\beta^{\prime}$-curves. Since $\delta \cap \beta_{1}$ contains only one point, namely the intersection that can be seen in Figures 4 and 5, the disc has to run through either $\mathcal{D}_{*}$ or $\mathcal{D}_{* *}$ (since $n_{z}(\phi)=0$ we cannot use the $\mathcal{D}_{z}$-region). But since we are moving from the $\delta$-part of $\beta_{1}^{\prime}$ to the $\beta$-part of $\beta_{1}^{\prime}$, we see that $n_{*}(\phi)<0$ or $n_{* *}(\phi)<0$, in contradiction to holomorphicity. So, there are just three choices for the $\beta$-boundary of a holomorphic disc.

(1) It starts at the $\delta$-part of $\beta_{1}^{\prime}$ and stays there.

(2) It starts at the $\beta$-part of $\beta_{1}^{\prime}$ and stays there.

(3) It starts at the $\beta$-part of $\beta_{1}^{\prime}$ and runs to the $\delta$-part of $\beta_{1}^{\prime}$ and stays there.

This immediately shows that

$$
\widehat{\mathrm{HF}}\left(Y^{\delta}\right)=H_{*}(\widehat{\mathrm{CF}}(\alpha, \beta) \oplus \widehat{\mathrm{CF}}(\alpha, \delta), \partial),
$$

where $\partial$ is of the form

$$
\left(\begin{array}{ll}
A & C \\
0 & B
\end{array}\right)
$$

If we perform a negative Dehn twist along $\delta$ in the situation indicated in Figure 4, we would connect $\mathcal{D}_{*}$ with $\mathcal{D}_{* *}$ and keep separate $\mathcal{D}_{w}$ and $\mathcal{D}_{z}$. Observe that we would have, a priori, no control of holomorphic discs like in the case of positive Dehn twists. To get back into business, in case of negative Dehn twists, we have to first isotope $\delta$ inside the page of the open book appropriately (see Section 4.2).

Proposition 4.4 Let $(\Sigma, \alpha, \beta)$ be a $\delta$-adapted Heegaard diagram of $Y$ and denote by $Y^{\delta}$ the manifold obtained from $Y$ by composing the gluing map, given by the attaching curves $\alpha, \beta$, with a positive Dehn twist along $\delta$ as indicated in Figure 5. Then the following holds:

$$
\widehat{\mathrm{HF}}\left(Y^{\delta}\right) \cong H_{*}\left(\widehat{\mathrm{CF}}(\alpha, \beta) \oplus \widehat{\mathrm{CF}}(\alpha, \delta), \partial^{f}\right),
$$

where $\partial^{f}$ is of the form

$$
\left(\begin{array}{cc}
\hat{\partial}_{\alpha \beta}^{w} & f \\
0 & \widehat{\partial}_{\alpha \delta}^{w}
\end{array}\right)
$$

with $f$ a chain map between $\left(\widehat{\mathrm{CF}}(\alpha, \delta), \widehat{\partial}_{\alpha \delta}^{w}\right)$ and $\left(\widehat{\mathrm{CF}}(\alpha, \beta), \widehat{\partial}_{\alpha \beta}^{w}\right)$. 
Proof There is a natural identification of intersection points

$$
\mathbb{T}_{\alpha} \cap \mathbb{T}_{\beta^{\prime}} \longleftrightarrow \mathbb{T}_{\alpha} \cap \mathbb{T}_{\beta} \sqcup \mathbb{T}_{\alpha} \cap \mathbb{T}_{\delta},
$$

that is, we get an isomorphism

$$
\epsilon: \widehat{\mathrm{CF}}\left(\alpha, \beta^{\prime}\right) \stackrel{\cong}{\longrightarrow} \widehat{\mathrm{CF}}(\alpha, \beta) \oplus \widehat{\mathrm{CF}}(\alpha, \delta)
$$

of modules. Pick an intersection point $x \in \mathbb{T}_{\alpha} \cap \mathbb{T}_{\beta^{\prime}}$ such that $\epsilon(x) \in \mathbb{T}_{\alpha} \cap \mathbb{T}_{\beta}$. Looking at the boundary

$$
\widehat{\partial}^{\delta} x=\sum_{y} \sum_{\phi} \# \widehat{\mathcal{M}}_{\phi} \cdot y
$$

we want to see that the moduli space of holomorphic discs connecting $x$ with an intersection $y \in \epsilon^{-1}\left(\mathbb{T}_{\alpha} \cap \mathbb{T}_{\delta}\right)$ is empty: Assume this were not the case. This means there were a holomorphic disc $\phi$ connecting $x$ with an element $y=\left(y_{1}, \ldots, y_{n}\right) \in$ $\epsilon^{-1}\left(\mathbb{T}_{\alpha} \cap \mathbb{T}_{\delta}\right)$. Observe that $y_{1}$ is a point in $\delta \cap \alpha_{1}$. Hence, $\mathcal{D}(\phi)$ includes $\mathcal{D}_{*}$ or $\mathcal{D}_{* *}$ since these are the only domains giving a connection between $\mathbb{T}_{\alpha} \cap \mathbb{T}_{\beta}$ and $\mathbb{T}_{\alpha} \cap \mathbb{T}_{\delta}$. Boundary orientations force the coefficient of $\phi$ at $\mathcal{D}_{*}$ or $\mathcal{D}_{* *}$ to be negative. Since holomorphic maps are orientation preserving, this cannot be the case. So, the point $x$ can be connected to points in $\epsilon^{-1}\left(\mathbb{T}_{\alpha} \cap \mathbb{T}_{\beta}\right)$ only.

Next observe that discs $\phi$ appearing in the sum (4-2) all have the property $n_{*}(\phi)=$ $n_{* *}(\phi)=0$. Indeed, suppose there were a disc $\phi$ with nonnegative intersection $n_{*}$ or $n_{* *}$. The $\beta$-boundary of $\phi$ starts at $x$ and runs through $\partial \mathcal{D}_{*}$ or $\partial \mathcal{D}_{* *}$. The disc $\phi$ is holomorphic, so, the $\beta$-boundary runs from the $\beta$-part to the $\delta$-part of $\mathbb{T}_{\beta^{\prime}}$. At the end of the $\beta$-boundary of $\phi$ the disc converges to a point in $\mathbb{T}_{\alpha} \cap \mathbb{T}_{\beta}$. Thus, the $\beta$ boundary of $\phi$ has to come back through either $\mathcal{D}_{*}$ or $\mathcal{D}_{* *}$. The boundary orientation would force $\phi$ to negatively intersect $\{*\} \times \operatorname{Sym}^{g-1}(\Sigma)$ or $\{* *\} \times \operatorname{Sym}^{g-1}(\Sigma)$. This cannot happen.

Denote by $[a, c]$ the small arc in $\beta_{1}^{\prime}$ running through Figure 6 and define $[b, d]$ analogously. All discs arising in the sum have boundary conditions in $\mathbb{T}_{\alpha}$ and

$$
\mathbb{T}_{\beta^{\prime}} \backslash\left\{\{[a, c] \sqcup[b, d]\} \times \beta_{2} \times \cdots \times \beta_{g}\right\} .
$$

Observe that $\mathbb{T}_{\beta^{\prime}} \backslash\left\{\{[a, c] \sqcup[b, d]\} \times \beta_{2} \times \cdots \times \beta_{g}\right\}$ has two components, one lying in $\mathbb{T}_{\beta}$ and one lying in $\mathbb{T}_{\delta}$. Since the $\beta$-boundary of the disc $\phi$ starts in, $\mathbb{T}_{\beta}$ it remains there all the time. Moreover, looking at discs $\phi$ in $(\Sigma, \alpha, \beta, z, w)$ with $n_{z}(\phi)=n_{w}(\phi)=0$, an analogous line of arguments as above shows that the $\beta$-boundary of these discs stays away from

$$
[a, b] \times \beta_{2} \times \cdots \times \beta_{g},
$$




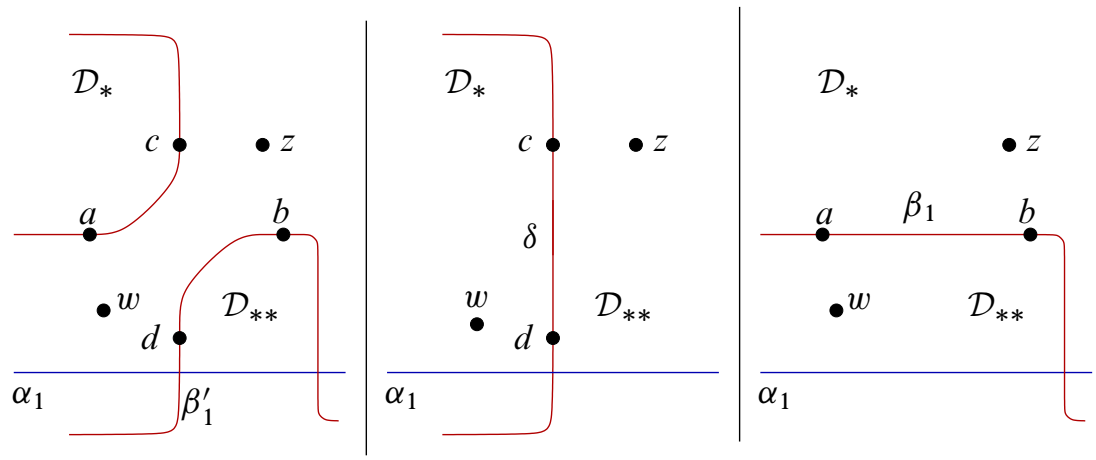

Figure 6: Picture of the three different boundary conditions arising in our discussion

where $[a, b]$ is the arc in $\beta$ pictured in the right of Figure 6 . Thus, the boundary conditions for discs connecting intersections $\mathbb{T}_{\alpha} \cap \mathbb{T}_{\beta}$ are the same in $\left(\Sigma, \alpha, \beta^{\prime}, z\right)$ and $(\Sigma, \alpha, \beta, z, w)$. Thus, we have

$$
\widehat{\partial}^{\delta} x=\widehat{\partial}_{\alpha \beta}^{w} x
$$

Now suppose that $x \in \epsilon^{-1}\left(\mathbb{T}_{\alpha} \cap \mathbb{T}_{\delta}\right)$. Then

$$
\widehat{\partial}^{\delta} x=\sum_{y} \sum_{\phi} \# \widehat{\mathcal{M}}_{\phi} \cdot y=\sum_{y \in \mathbb{T}_{\alpha} \cap \mathbb{T}_{\delta}} \sum_{\phi} \# \widehat{\mathcal{M}}_{\phi} \cdot y+\sum_{z \in \mathbb{T}_{\alpha} \cap \mathbb{T}_{\beta}} \sum_{\phi} \# \widehat{\mathcal{M}}_{\phi} \cdot z .
$$

With an analogous line of arguments as above we see that the first sum counts discs with $n_{*}=n_{* *}=n_{z}=0$ only. The triviality of these intersection numbers and holomorphicity implies that the discs have boundary conditions in $\mathbb{T}_{\alpha}$ and

$$
\mathbb{T}_{\beta^{\prime}} \backslash\left\{\{[a, c] \sqcup[b, d]\} \times \beta_{2} \times \cdots \times \beta_{g}\right\} .
$$

As mentioned above this set has two components where one of them lies in $\mathbb{T}_{\delta}$. The $\beta$-boundary of $\phi$ starts in $\mathbb{T}_{\delta}$ and therefore remains there all the time. Again, we see that discs connecting intersection points $\mathbb{T}_{\alpha} \cap \mathbb{T}_{\delta}$ in $\left(\Sigma, \alpha, \beta^{\prime}, z\right)$ and $(\Sigma, \alpha, \delta, z, w)$ have to fulfil identical boundary conditions. Thus, the moduli spaces are isomorphic. This shows the equality

$$
\widehat{\partial}^{\delta} x=\widehat{\partial}_{\alpha \delta}^{w} x+\sum_{z \in \mathbb{T}_{\alpha} \cap \mathbb{T}_{\beta}} \sum_{\phi} \# \widehat{\mathcal{M}}_{\phi} \cdot z .
$$

In the right sum we only count discs where $n_{*} \neq 0$ or $n_{* *} \neq 0$. We will denote this right sum with $f(x)$. We have to see that $f$ defines a chain map

$$
f:\left(\widehat{\mathrm{CF}}(\alpha, \delta), \widehat{\partial}_{\alpha \delta}^{w}\right) \longrightarrow\left(\widehat{\mathrm{CF}}(\alpha, \beta), \widehat{\partial}_{\alpha \beta}^{w}\right) .
$$


This can be proved in two ways: We know that $\partial^{\delta}=\partial_{\alpha \beta}^{w}+\partial_{\alpha \delta}^{w}+f$. Hence, $f$ is a sum of three boundaries. The equality $0=\left(\partial^{\delta}\right)^{2}$ implies that $f$ is a chain map (see Lemma 3.1). The second way is to test the chain map property directly. To do so, pick a generator $y \in \mathbb{T}_{\alpha} \cap \mathbb{T}_{\beta^{\prime}}$ lying in the preimage of $\mathbb{T}_{\alpha} \cap \mathbb{T}_{\delta}$ under $\epsilon$. Observe that $\left(\widehat{\partial}_{\alpha \beta}^{w} \circ f-f \circ \widehat{\partial}_{\alpha \delta}^{w}\right)(x)$ equals

$$
\sum_{z \in \mathbb{T}_{\alpha} \cap \mathbb{T}_{\delta}}\left(\sum_{\left(y, \phi_{2}, \phi_{1}\right)} \# \widehat{\mathcal{M}}\left(\phi_{2}\right) \# \widehat{\mathcal{M}}\left(\phi_{1}\right)-\sum_{\left(y^{\prime}, \phi_{2}^{\prime}, \phi_{1}^{\prime}\right)} \# \widehat{\mathcal{M}}\left(\phi_{2}^{\prime}\right) \# \widehat{\mathcal{M}}\left(\phi_{1}^{\prime}\right)\right) \cdot z=\sum_{z \in \mathbb{T}_{\alpha} \cap \mathbb{T}_{\delta}} c(x, z) \cdot z,
$$

where the first sum in the definition of $c(x, z)$ goes over elements $\left(y, \phi_{2}, \phi_{1}\right)$ in the set $\mathbb{T}_{\alpha} \cap \mathbb{T}_{\beta} \times \pi_{2}(y, z) \times \pi_{2}(x, y)$ with $\mu\left(\phi_{2}\right)=\mu\left(\phi_{1}\right)=1$, and the second sum goes over $\left(y^{\prime}, \phi_{2}^{\prime}, \phi_{1}^{\prime}\right) \in \mathbb{T}_{\alpha} \cap \mathbb{T}_{\delta} \times \pi_{2}(y, z) \times \pi_{2}(x, y)$ with $\mu\left(\phi_{2}^{\prime}\right)=\mu\left(\phi_{1}^{\prime}\right)=1$. Furthermore, look at the boundary of a moduli space $\widehat{\mathcal{M}}(\phi)$ connecting a point in $\mathbb{T}_{\alpha} \cap \mathbb{T}_{\delta}$ with a point in $\mathbb{T}_{\alpha} \cap \mathbb{T}_{\beta}$ with $\mu(\phi)=2$. Observe that we do not have to take care of boundary degenerations or spheres bubbling off since we are looking for maps with $n_{z}=0$ (see Ozsváth and Szabó [23]). The only phenomenon appearing at the boundary is breaking. The boundary of $\widehat{\mathcal{M}}(\phi)$ is modelled on

$$
\bigsqcup_{\phi_{1} * \phi_{2}=\phi} \widehat{\mathcal{M}}\left(\phi_{1}\right) \times \widehat{\mathcal{M}}\left(\phi_{2}\right) .
$$

There are two cases. Either $n_{*}\left(\phi_{1}\right)=n_{*}(\phi)$ or $n_{*}\left(\phi_{2}\right)=n_{*}(\phi)$ (the discussion for $n_{* *}$ is analogous):

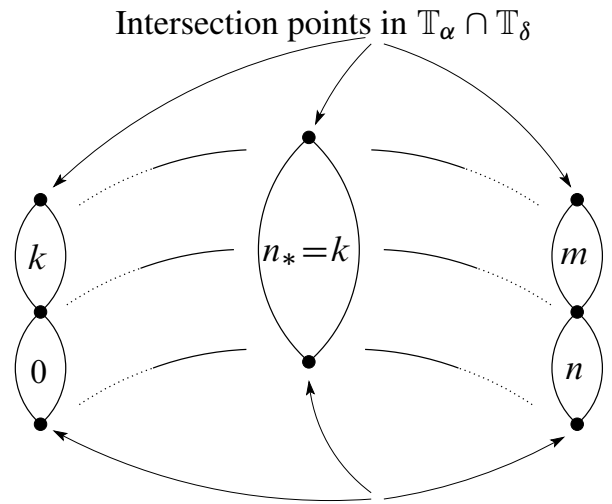

Intersection points in $\mathbb{T}_{\alpha} \cap \mathbb{T}_{\beta}$

Figure 7: Here we figure a moduli space with $\mu=2$ and its possible ends

To prove this, we have to show that a given family of discs $\phi_{n}$ in $\widehat{\mathcal{M}}(\phi)$ cannot converge to a broken disc $\phi_{1} * \phi_{2}$ with $n=n_{*}\left(\phi_{1}\right) \neq 0$ and $m=n_{*}\left(\phi_{2}\right) \neq 0$. Figure 7 represents 
a moduli space of discs with $\mu=2$ and $n_{*}\left(\phi_{n}\right)=k$. We know that $n+m=k$, since intersection numbers behave additively under concatenation. Assume that $n, m$ were both non-zero: Since $n$ is non-zero, we know that $\phi_{1}$ connects a point in $\mathbb{T}_{\alpha} \cap \mathbb{T}_{\delta}$ with one in $\mathbb{T}_{\alpha} \cap \mathbb{T}_{\beta}$. The bottom intersection is a $\mathbb{T}_{\alpha} \cap \mathbb{T}_{\beta}$-intersection, since $\phi_{n}$ connects $\mathbb{T}_{\alpha} \cap \mathbb{T}_{\delta}$ with an $\mathbb{T}_{\alpha} \cap \mathbb{T}_{\beta}$-intersection by assumption. Hence, $\phi_{2}$ connects a point of $\mathbb{T}_{\alpha} \cap \mathbb{T}_{\beta}$ with a point in $\mathbb{T}_{\alpha} \cap \mathbb{T}_{\beta}$ and runs through the domain $\mathcal{D}_{*}$. This is simply not possible because of orientation reasons. Thus, either $n_{*}\left(\phi_{1}\right)=k$ and $n_{*}\left(\phi_{2}\right)=0$ or $n_{*}\left(\phi_{1}\right)=0$ and $n_{*}\left(\phi_{2}\right)=k$. This means the ends of $\widehat{\mathcal{M}}(\phi)$ precisely look like

$$
\left(\bigsqcup_{\phi_{2} * \phi_{1}=\phi} \widehat{\mathcal{M}}\left(\phi_{2}\right)^{*} \times \widehat{\mathcal{M}}\left(\phi_{1}\right)\right) \sqcup\left(\bigsqcup_{\phi_{2}^{\prime} * \phi_{1}^{\prime}=\phi} \widehat{\mathcal{M}}\left(\phi_{2}\right) \times \widehat{\mathcal{M}}\left(\phi_{1}\right)^{*}\right),
$$

where $*$ means that the associated discs have non-trivial intersection number $n_{*}$ or $n_{* *}$. Now consider the union of moduli spaces of discs connecting the point $x$ and $z$ with Maslov index 2. According to our discussion, the ends look like

$$
\left(\bigsqcup_{\left(y, \phi_{2}, \phi_{1}\right)} \widehat{\mathcal{M}}\left(\phi_{2}\right) \times \widehat{\mathcal{M}}\left(\phi_{1}\right)^{*}\right) \sqcup\left(\bigsqcup_{\left(y^{\prime}, \phi_{2}^{\prime}, \phi_{1}^{\prime}\right)} \widehat{\mathcal{M}}\left(\phi_{2}^{\prime}\right)^{*} \times \widehat{\mathcal{M}}\left(\phi_{1}^{\prime}\right)\right),
$$

where the first union goes over $\left(y, \phi_{2}, \phi_{1}\right) \in \mathbb{T}_{\alpha} \cap \mathbb{T}_{\beta} \times \pi_{2}(y, z) \times \pi_{2}(x, y)$ with $\mu\left(\phi_{2}\right)=\mu\left(\phi_{1}\right)=1$ and the second union goes over $\left(y^{\prime}, \phi_{2}^{\prime}, \phi_{1}^{\prime}\right) \in \mathbb{T}_{\alpha} \cap \mathbb{T}_{\delta} \times \pi_{2}(y, z) \times$ $\pi_{2}(x, y)$ with $\mu\left(\phi_{2}^{\prime}\right)=\mu\left(\phi_{1}^{\prime}\right)=1$. Hence, the coefficients $c(x, z)$ all vanish, proving the theorem.

An immediate, simple algebraic consequence (see Section 3) of this description is the following Corollary.

Corollary 4.5 Let $K \subset Y$ be the knot determined by $\delta$. Then there is a long exact sequence

$$
\ldots \stackrel{\partial_{*}}{\longrightarrow} \widehat{\operatorname{HFK}}(Y, K) \stackrel{\Gamma_{1}}{\longrightarrow} \widehat{\mathrm{HF}}\left(Y_{-1}(K)\right) \stackrel{\Gamma_{2}}{\longrightarrow} \widehat{\mathrm{HFK}}\left(Y_{0}(K), \mu\right) \stackrel{\partial_{*}}{\longrightarrow} \ldots
$$

with $\partial_{*}=-f_{*}$ where $f$ is the map defined in the proof of Proposition 4.4. The knot $\mu$ denotes a meridian of $K$.

Proof With Proposition 4.4 we see that $\widehat{\mathrm{HF}}\left(Y^{\delta}\right)$ fulfils the assumptions of Lemma 3.1 and therefore Lemma 3.2 applies. Finally, we apply Proposition 2.4 to identify $H_{*}\left(\widehat{\mathrm{CF}}, \widehat{\partial}^{w}\right)$ with the respective knot Floer homology. It is easy to observe that with respect to the framing induced by the open book the manifold $Y^{\delta}$ equals $Y_{-1}(K)$, that is, the result of $(-1)$-surgery along the knot $K$. We obtain the sequence

$$
\ldots \stackrel{\partial_{*}}{\longrightarrow} \widehat{\operatorname{HFK}}(Y, K) \stackrel{\Gamma_{1}}{\longrightarrow} \widehat{\mathrm{HF}}\left(Y_{-1}(K)\right) \stackrel{\Gamma_{2}}{\longrightarrow} \widehat{\mathrm{HFK}}\left(Y_{\alpha \delta}, K_{2}\right) \stackrel{\partial_{*}}{\longrightarrow} \ldots,
$$


where $\left(Y_{\alpha \delta}, K_{2}\right)$ is the pair given by the data $(\Sigma, \alpha, \delta, z, w)$. It is easy to see that the pair $(w, z)$ in the diagram $(\Sigma, \alpha, \delta)$ determines $\beta_{1}$ up to orientation, that is, the attaching circle $\beta_{1}$ interpreted as a knot in $Y_{\alpha \delta}$. This attaching circle $\beta_{1}$ is a meridian for a tubular neighborhood $\mu$ of $K$ in $Y$. Finally, we have to see that $Y_{\alpha \delta}$ equals the 0 -surgery along $K$ with respect to the framing induced by the open book. This is straightforward.

A few words about admissibility: The reader may have noticed that we did not say anything about admissibility of the Heegaard diagram $(\Sigma, \alpha, \delta, z, w)$, but nonetheless talk about the knot Floer homology $\widehat{\mathrm{HFK}}\left(Y_{\alpha \delta}, K_{2}\right)$ induced by this diagram. We could restrict to just saying we take the homology induced by the data. The respective boundary operator is well defined (finite sum) since $\widehat{\partial}^{\delta}$ is. However, we would like to remark that the diagram $(\Sigma, \alpha, \delta, z, w)$ is always admissible in a relaxed sense. We may relax the weak-admissibility condition imposed by Ozsváth and Szabó for the definition of knot Floer homology to the extreme weak-admissibility condition given in Definition 2.5. The diagram $(\Sigma, \alpha, \delta, z, w)$ is always extremely weakly-admissible: Let $\mathcal{D}$ be a non-trivial periodic domain with $n_{w}(\mathcal{D})=0$ (see Section 2.2.2) and let $s$ be an arbitrary $\operatorname{Spin}^{c}$-structure such that $\left\langle c_{s}(s), \mathcal{H}(\mathcal{D})\right\rangle=0$. By definition of the boundary, $\partial \mathcal{D}$ can be written as

$$
\partial \mathcal{D}=\sum_{i \geq 1} \lambda_{i} \alpha_{i}+\kappa_{1} \delta+\sum_{j \geq 2} \kappa_{j} \beta_{j}
$$

Assuming that $\lambda_{i} \neq 0$ for a $i \geq 2$ or $\kappa_{j} \neq 0$ for a $j \geq 2$, we see that $\mathcal{D}$ has both positive and negative coefficients due to the fact that $\partial \mathcal{D}$ runs through a configuration like given in Figure 1. Thus, let us assume that $\lambda_{i}$ and $\kappa_{j}$ would vanish, for all $i, j \geq 2$. The boundary of $\mathcal{D}$ could be written as

$$
\partial \mathcal{D}=\lambda_{1} \alpha_{1}+\kappa_{1} \delta .
$$

However, $\kappa_{1}$ has to vanish, since $\delta$ runs through $\partial \overline{\mathcal{D}_{w}} \cap \partial \overline{\mathcal{D}_{z}}$ (see Figure 6). Finally, we get that $\partial \mathcal{D}=\lambda_{1} \alpha_{1}$. Examining the middle part of Figure 6 we see that the part of $\alpha_{1}$ which is at the right of $\delta$ is surrounded by the region $\mathcal{D}_{z}$. Thus, $\lambda_{1}=0$.

With help of the geometric realization of the $\Lambda^{*}\left(H_{1} /\right.$ Tor $)$-module structure given by Ozsváth and Szabó [23] we can easily prove the following proposition.

Proposition 4.6 The maps $\Gamma_{1}$ and $\Gamma_{2}$ from the exact sequence of Corollary 4.5 respect the $\wedge^{*}\left(H_{1} /\right.$ Tor $)$-module structure of the Heegaard Floer groups in the following sense. Let $\gamma \subset \Sigma$ be a curve. Then the following identities hold:

$$
A_{[\gamma]_{Y} \delta}^{Y^{\delta}}\left(\Gamma_{1}(x)\right)=\Gamma_{1}\left(A_{[\gamma]_{Y}}^{Y}(x)\right) \quad \Gamma_{2}\left(A_{[\gamma]_{Y} \delta}^{Y^{\delta}}(x)\right)=A_{[\gamma]_{Y_{\alpha \delta}}}^{Y_{\alpha \delta}}\left(\Gamma_{2}(x)\right)
$$


Proof Recall the geometric realization of the $\bigwedge^{*}\left(H_{1} /\right.$ Tor $)$-module structure. Given a point $x \in \mathbb{T}_{\alpha} \cap \mathbb{T}_{\beta} \subset \mathbb{T}_{\alpha} \cap \mathbb{T}_{\beta^{\prime}}$ (see the proof of Proposition 4.4 for the appropriate identification), by definition

$$
A_{[\gamma]_{Y^{\delta}}}^{Y^{\delta}}(x)=\sum_{y} \sum_{\phi \in H(x, y, 1)} a(\gamma, \phi) \cdot y,
$$

where $H(x, y, 1) \subset \pi_{2}(x, y)$ is the set of Whitney discs with $n_{z}=0$ and $\mu=1$. Furthermore,

$$
a(\gamma, \phi)=\# \widehat{\mathcal{M}}_{\phi} \cdot \#\left(u(\{-1\} \times \mathbb{R}), \gamma \times \operatorname{Sym}^{g-1}(\Sigma)\right)_{\mathbb{T}_{\alpha}}
$$

where the right factor denotes the intersection number of $u(\{-1\} \times \mathbb{R})$ and $\gamma \times$ Sym $^{g-1}(\Sigma)$ inside $\mathbb{T}_{\alpha}$. Fixing another point $y \in \mathbb{T}_{\alpha} \cap \mathbb{T}_{\beta}$, recall that these points are connected by $\widehat{\partial}_{\alpha \beta}^{w}$ if and only if they are connected by $\widehat{\partial}^{\delta}$. Moreover, there is an identification of the respective moduli spaces. Thus, fixing a disc $\phi$ connecting these points (in $\alpha \beta^{\prime}$ ), we know - since $n_{z}(\phi)=0$ - that $\phi$ connects these intersection points in the $\alpha \beta$-diagram, too. Denoting by $[\phi]$ its class in $\pi_{2}$, we see that

$$
\# \widehat{\mathcal{M}}_{[\phi]}^{\alpha \beta}=\# \widehat{\mathcal{M}}_{[\phi]}^{\alpha \beta^{\prime}} .
$$

Moreover, the intersection number in $\mathbb{T}_{\alpha}$ used to define $a(\gamma,[\phi])$ coincides for both diagrams since $\phi$ is a common representative. Thus, we see that

$$
a^{Y^{\delta}}(\gamma,[\phi])=a^{Y}(\gamma,[\phi]) .
$$

Recall that there are no connections from $\mathbb{T}_{\alpha} \cap \mathbb{T}_{\beta}$-intersections to a $\mathbb{T}_{\alpha} \cap \mathbb{T}_{\delta}$ intersection in the $\alpha, \beta^{\prime}$-diagram. Hence, the first equality given in the proposition follows.

To show the second, fix a point $x \in \mathbb{T}_{\alpha} \cap \mathbb{T}_{\delta} \subset \mathbb{T}_{\alpha} \cap \mathbb{T}_{\beta^{\prime}}$. Use the same line of arguments as above to show that the following identity holds:

$$
\begin{array}{r}
A_{[\gamma]_{Y} \delta}^{Y^{\delta}}(x)=\sum_{y} \sum_{\phi \in H(x, y, 1)} a^{Y^{\delta}}(\gamma, \phi) \cdot y+\sum_{z} \sum_{\psi \in H(x, z, 1)} a^{Y^{\delta}}(\gamma, \psi) \cdot z \\
=A_{[\gamma]_{Y_{\alpha \delta}}}^{Y_{\alpha \delta}}(x)+\sum_{z} \sum_{\psi \in H(x, z, 1)} a^{Y^{\delta}}(\gamma, \psi) \cdot z
\end{array}
$$

The second sum is an element in $\widehat{\mathrm{CF}}(\Sigma, \alpha, \beta, z, w)$. Recall that $\Gamma_{2}$ is induced by the projection onto $\widehat{\mathrm{CF}}(\Sigma, \alpha, \delta, z, w)$. Hence, the second sum cancels when projected under the map $\Gamma_{2}$. The second equality of the proposition follows.

In Section 5 we will derive suitable naturality properties of the sequence to show that the maps involved in the sequences are indeed topological. We will be interested in the 
maps denoted by $\Gamma_{1}$ since these are directly related to the surgery represented by the Dehn twist.

Remark 2 A result similar to Corollary 4.5 and Corollary 4.8 can be derived that involves the groups $\mathrm{HFK}^{-}$using methods applied in this paragraph.

\subsection{Negative Dehn twists}

The approach for negative Dehn twists is pretty much the same as for positive Dehn twists. In Section 4.1 we already mentioned that the situation indicated in Figure 4 is not suitable for performing negative Dehn twists. Performing a negative twist, we could not make an a priori statement about what generators can be connected by holomorphic discs like we did in Section 4.1. To get back into business we just need to isotope the curve $\delta$ inside the page a bit (or equivalently isotope some of the attaching circles). Figure 8 indicates a possible perturbation suitable for our purposes. Comparing Figures 5 and 8 we see that we isotoped the curve $\delta$ a bit. Observe that

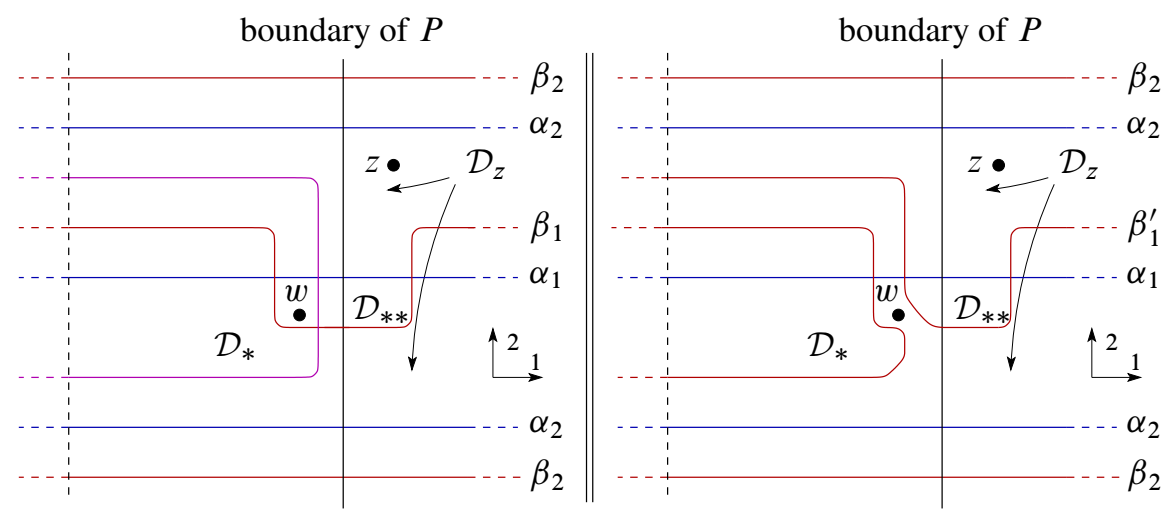

Figure 8: Before and after a negative Dehn twist along $\delta$

with this perturbation done, we again can read off the behavior of holomorphic discs like in Section 4.1 (carry over the discussion of Section 4.1 to this situation). As a consequence, the following proposition can be proved. The proof of Proposition 4.4 carries over verbatim to a proof of Proposition 4.7.

Proposition 4.7 Let $(\Sigma, \alpha, \beta)$ be a $\delta$-adapted Heegaard diagram of $Y$ and denote by $Y^{\delta}$ the manifold obtained from $Y$ by composing the gluing map, given by the attaching curves $\alpha, \beta$, with a negative Dehn twist along $\delta$ as hinted in Figure 8 . Then we have

$$
\widehat{\mathrm{HF}}\left(Y^{\delta}\right) \cong H_{*}\left(\widehat{\mathrm{CF}}(\alpha, \beta) \oplus \widehat{\mathrm{CF}}(\alpha, \delta), \partial^{f}\right),
$$


where $\partial^{f}$ is of the form

$$
\left(\begin{array}{cc}
\widehat{\partial}_{\alpha \beta}^{w} & 0 \\
f & \widehat{\partial}_{\alpha \delta}^{w}
\end{array}\right)
$$

with $f$ a chain map between $\left(\widehat{\mathrm{CF}}(\alpha, \delta), \widehat{\partial}_{\alpha \delta}^{w}\right)$ and $\left(\widehat{\mathrm{CF}}(\alpha, \beta), \widehat{\partial}_{\alpha \beta}^{w}\right)$.

Corollary 4.8 Let $K \subset Y$ be the knot determined by $\delta$. Then there is a long exact sequence

$$
\ldots \stackrel{\partial_{*}}{\longrightarrow} \widehat{\operatorname{HFK}}\left(Y_{0}(K), \mu\right) \stackrel{\Gamma_{2}}{\longrightarrow} \widehat{\mathrm{HF}}\left(Y_{+1}(K)\right) \stackrel{\Gamma_{1}}{\longrightarrow} \widehat{\operatorname{HFK}}(Y, K) \stackrel{\partial_{*}}{\longrightarrow} \ldots
$$

with $\partial_{*}=-f_{*}$ where $f$ is the map defined in the proof of Proposition 4.7. The knot $\mu$ denotes a meridian of $K$. Moreover, identities hold similar to those given in Proposition 4.6.

\section{Invariance}

Our goal in this paragraph is to show that the map $\Gamma_{1}$ in the sequences introduced are topological, that is, they just depend on the cobordism associated to the surgery represented by the Dehn twist. To do that, we have to generalize our approach a bit and try to see that everything we have done, especially the proof of Proposition 4.4, works without using a Heegaard diagram that is necessarily induced by an open book. Obviously, the geometric situation given in Figure 6 builds the foundation of the proof. To clarify the situation, look at Figure 9.

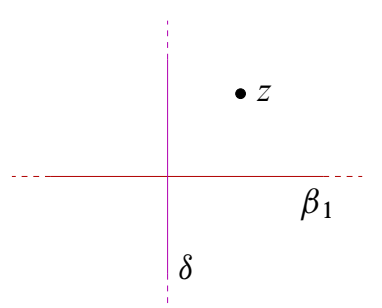

Figure 9: The important geometric configuration

We, for the moment, stick to the notation of Section 4 . We need the curve $\delta$ to intersect $\beta_{1}$ once, transversely and to be disjoint from the other $\beta$-circles. In addition, the top right domain at the point $\delta \cap \beta_{1} \in \Sigma$ has to contain the base point $z$ (see Figure 9). Given this configuration, the proof of Proposition 4.4 applies. The situation illustrated, does not occur exclusively when the Heegaard diagram is induced by an open book. 

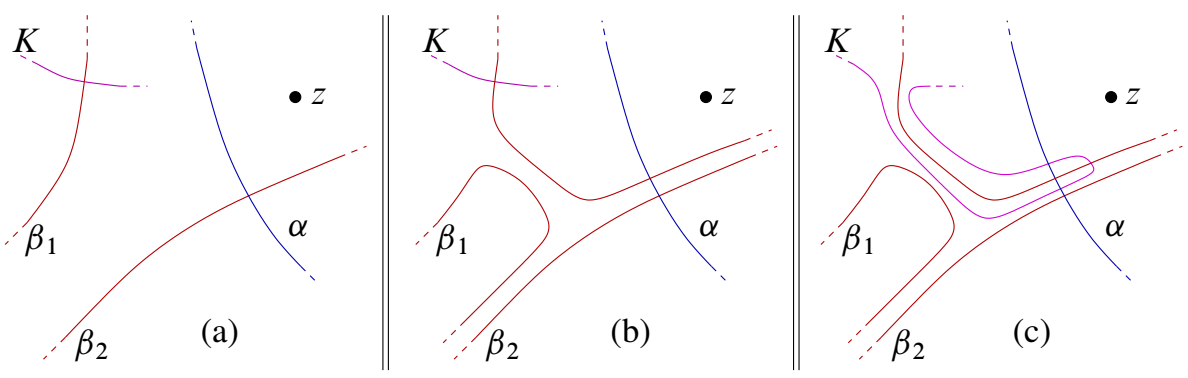

Figure 10: Preparation of the Heegaard diagram

Given a Heegaard diagram subordinate to a knot $K$, we can isotope the knot $K$ onto the Heegaard surface. The isotoped knot intersects just one $\beta$-circle once, transversely. Without loss of generality $K$ intersects $\beta_{1}$. To generate a geometric configuration like indicated in Figure 9, we may isotope the knot again to move the intersection $\beta_{1} \cap K$ to lie next to a $\mathcal{D}_{z}$-region: Cutting the $\alpha$-circles out of the Heegaard surface, we obtain a sphere with holes. The region $\mathcal{D}_{z}$ is a region in this sphere. Either $\mathcal{D}_{z}$ is the whole sphere with holes or not. In case it is the whole sphere all the $\beta$-circles touch the region $\mathcal{D}_{z}$ and we are done. In case $\mathcal{D}_{z}$ is not the whole sphere, there has to be at least one $\beta$-circle touching the boundary of $\mathcal{D}_{z}$. If $\beta_{1}$ touches the boundary of $\mathcal{D}_{z}$, we are done. If $\beta_{1}$ does not touch the boundary of $\mathcal{D}_{z}$, we obtain a configuration like indicated in part (a) of Figure 10. Without loss of generality we assume that $\beta_{2}$ touches $\mathcal{D}_{z}$. Note that it not possible for $\beta_{2}$ to separate $\mathcal{D}_{z}$ from $\beta_{1}$, since the complement of the $\beta$-circles in $\Sigma$ is connected. We are allowed to slide $\beta_{1}$ over this $\beta$-circle (see part (b) of Figure 10). After the handle slide there is a small arc $a$ inside $\beta_{1}$ touching $\mathcal{D}_{z}$. By a small isotopy of the knot $K$ we can move the intersection point $K \cap \beta_{1}$ along the new $\beta_{1}$-circle until it enters the arc $a$ (see part (c) of Figure 10).

Care has to be taken of the surgery framing. Here, we stick to surgeries or to framed knots $K$ such that there exists a subordinate Heegaard diagram with the framing induced by the Heegaard surface coinciding with the framing of the knot. Evidence indicate that every framing can be realized in this way.

We saw that our discussion from the last paragraph can be carried over to a more general situation. We, indeed, do not need the Heegaard diagram to be induced by an open book. So far, we restricted the discussion to Heegaard diagrams induced by open books, since we are interested in applications to the contact geometric parts of the theory, which makes a discussion of this class of diagrams inevitable.

Given two Heegaard diagrams subordinate to a pair $(Y, \delta)$, we transform the one diagram into the other by the moves introduced in Lemma 2.3. These moves respect 
the knot complement of $\delta$. The goal is to show that each move preserves the exact sequence and the maps inherited. In the following we will call Heegaard diagrams, realizing a geometric situation as given in Figure 6 for a knot $\delta, \delta$-suitable.

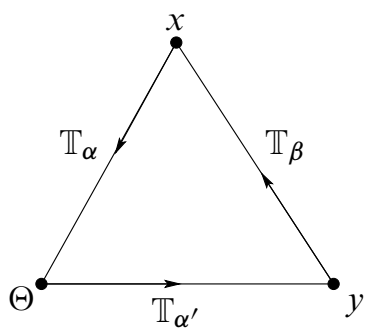

Figure 11: Triangles that have to be counted for handle slides among the $\alpha$-curves

We begin showing invariance under handle slides among the $\alpha$-curves. Although used in some papers it was never explicitly mentioned which triangles are counted for handle slides among the $\alpha$-curves. Given a Heegaard diagram $(\Sigma, \alpha, \beta)$, denote by $\alpha^{\prime}$ the attaching circles obtained by a handle slide among the $\alpha$-curves. The associated map between the respective homologies counts holomorphic triangles with boundary conditions in $\alpha, \alpha^{\prime}$ and $\beta$. Figure 11 pictures a Whitney triangle connecting a point $x \in \mathbb{T}_{\alpha} \cap \mathbb{T}_{\beta}$ with a point $y \in \mathbb{T}_{\alpha^{\prime}} \cap \mathbb{T}_{\beta}$. Observe that in this situation $\Theta$ is a top-dimensional generator of $\widehat{\mathrm{HF}}\left(\alpha^{\prime}, \alpha\right)$ (note the order of the attaching circles). To not confuse the maps induced by handle slides among the $\alpha$-circles with the maps induced by handle slides among the $\beta$-circles, we introduce the following notation: let us denote by $\Gamma_{\alpha, \alpha^{\prime} ; \beta}$ the map induced by a handle slide among the $\alpha$-circles (like indicated above) and by $\Gamma_{\alpha ; \beta, \beta^{\prime}}$ the map induced by a handle slide among the $\beta$-circles.

Proposition 5.1 Let $(\Sigma, \alpha, \beta, z)$ be a $\delta$-suitable Heegaard diagram and $\left(\Sigma, \alpha^{\prime}, \beta, z\right)$ be obtained by a handle slide of one of the $\alpha_{i}$. Denote by

$$
\begin{aligned}
\Gamma_{\alpha, \alpha^{\prime} ; \beta}^{w}: & \widehat{\mathrm{CFK}}(\Sigma, \alpha, \beta, z, w) \longrightarrow \widehat{\mathrm{CFK}}\left(\Sigma, \alpha^{\prime}, \beta, z, w\right) \\
\Gamma_{\alpha, \alpha^{\prime} ; \delta}^{w}: & \widehat{\mathrm{CFK}}(\Sigma, \alpha, \delta, z, w) \longrightarrow \widehat{\mathrm{CFK}}\left(\Sigma, \alpha^{\prime}, \delta, z, w\right) \\
\Gamma_{\alpha, \alpha^{\prime} ; \beta^{\prime}}: & \widehat{\mathrm{CF}}\left(\Sigma, \alpha, \beta^{\prime}, z\right) \longrightarrow \widehat{\mathrm{CF}}\left(\Sigma, \alpha^{\prime}, \beta^{\prime}, z\right)
\end{aligned}
$$

the induced maps. These maps induce a commutative diagram with exact rows $\ldots \stackrel{\partial_{*}}{\longrightarrow} \widehat{\operatorname{HFK}}(\Sigma, \alpha, \beta, z, w) \stackrel{\Gamma_{1}}{\longrightarrow} \widehat{\mathrm{HF}}\left(\Sigma, \alpha, \beta^{\prime}, z\right) \stackrel{\Gamma_{2}}{\longrightarrow} \widehat{\mathrm{HFK}}(\Sigma, \alpha, \delta, z, w) \stackrel{\partial_{*}}{\longrightarrow} \ldots$

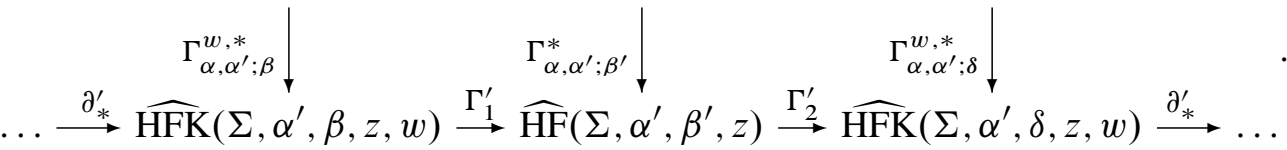


Proof The proof of this proposition is quite similar to the proof of Proposition 4.4. To keep the exposition efficient, we do not point out all details here. Start looking at the map $\Gamma_{\alpha, \alpha^{\prime} ; \beta^{\prime}}$. It is defined by counting triangles with boundary conditions in $\mathbb{T}_{\alpha}$, $\mathbb{T}_{\alpha^{\prime}}, \mathbb{T}_{\beta^{\prime}}$.
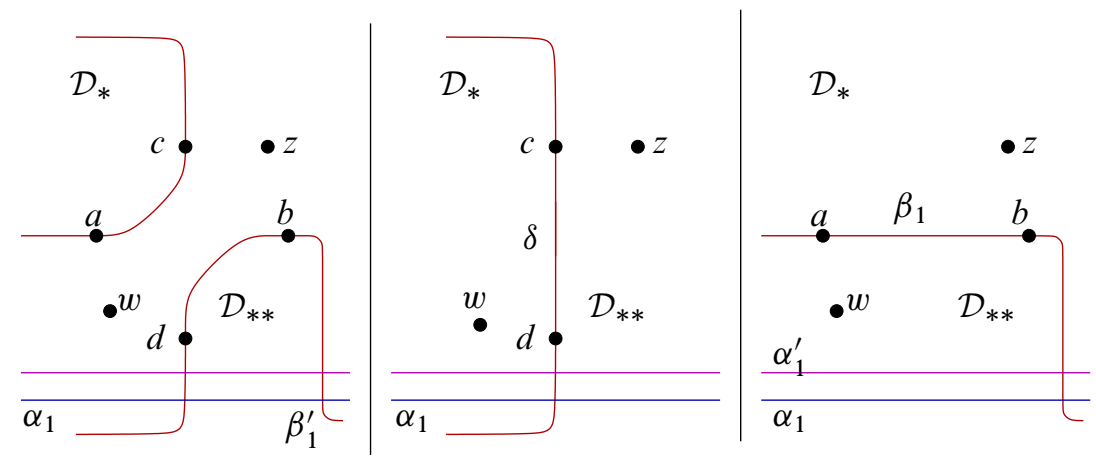

Figure 12: Picture of the three different boundary conditions arising in our discussion

Figure 12 illustrates the boundary conditions and how they look like near the region where the Dehn twist is performed. Analogous to the discussion in the proof of Proposition 4.4 the picture shows that

$$
\Gamma_{\alpha, \alpha^{\prime} ; \beta^{\prime}}=\left(\begin{array}{cc}
\Gamma_{\alpha, \alpha^{\prime} ; \beta}^{w} & \bar{\Gamma} \\
0 & -\Gamma_{\alpha, \alpha^{\prime} ; \delta}^{w}
\end{array}\right),
$$

where $\bar{\Gamma}$ is a map defined by counting triangles that connect $\mathbb{T}_{\alpha^{\prime}} \cap \mathbb{T}_{\delta}$-intersections with $\mathbb{T}_{\alpha} \cap \mathbb{T}_{\beta}$-intersections. This immediately shows commutativity of the first two boxes, that is,

$$
\begin{aligned}
& \Gamma_{\alpha, \alpha^{\prime} ; \beta^{\prime}}^{*} \circ \Gamma_{1}=\Gamma_{1}^{\prime} \circ \Gamma_{\alpha, \alpha^{\prime} ; \beta}^{w, *} \\
& \Gamma_{2}^{\prime} \circ \Gamma_{\alpha, \alpha^{\prime} ; \beta^{\prime}}^{*}=-\Gamma_{\alpha, \alpha^{\prime} ; \delta}^{w, *} \circ \Gamma_{1} .
\end{aligned}
$$

It remains to show that

$$
\Gamma_{\alpha, \alpha^{\prime} ; \beta}^{w, *} \circ \partial_{*}=\partial_{*}^{\prime} \circ-\Gamma_{\alpha, \alpha^{\prime} ; \delta}^{w, *} .
$$

Recall that $\partial_{*}$ equals the map $f$ in the definition of the boundary $\hat{\partial}^{\delta}$. These were defined by counting discs with $n_{*} \neq 0$ or $n_{* *} \neq 0$. Look at the following expression

$$
\Gamma_{\alpha, \alpha^{\prime} ; \beta}^{w, *} \circ f_{*}+f_{*}^{\prime} \circ \Gamma_{\alpha, \alpha^{\prime} ; \delta}^{w,}
$$

The strategy to show its vanishing is analogous to the discussion of the chain mapproperty of $f$ in the proof of Proposition 4.4. There are two ways to see this: Recall 
that $\Gamma_{\alpha, \alpha^{\prime} ; \beta^{\prime}}$ is a chain map. Hence, with the representation of $\widehat{\partial}^{\delta}$ given in Proposition 4.4 , this means that

$$
f^{\prime} \circ \Gamma_{\alpha, \alpha^{\prime} ; \delta}^{w}+\Gamma_{\alpha, \alpha^{\prime} ; \beta}^{w} \circ f=\widehat{\partial}_{\alpha^{\prime} \beta}^{w} \circ \bar{\Gamma}+\bar{\Gamma} \circ \widehat{\partial}_{\alpha^{\prime} \delta}^{w} .
$$

Thus,

$$
\begin{aligned}
0 & =\left(f^{\prime} \circ \Gamma_{\alpha, \alpha^{\prime} ; \delta}^{w}+\Gamma_{\alpha, \alpha^{\prime} ; \beta}^{w} \circ f\right)_{*} \\
& =f_{*}^{\prime} \circ \Gamma_{\alpha, \alpha^{\prime} ; \delta}^{w, *}+\Gamma_{\alpha, \alpha^{\prime} ; \beta}^{w, *} \circ f_{*}
\end{aligned}
$$

since all maps involved are chain maps. Hence, the third box commutes, too. Alternatively, look at the ends of the moduli spaces of Whitney triangles with boundary conditions in $\mathbb{T}_{\alpha}, \mathbb{T}_{\alpha^{\prime}}, \mathbb{T}_{\beta^{\prime}}$ with Maslov index 1 and non-trivial intersection number $n_{*}$ or $n_{* *}$. The ends look like given in Figure 13. There are three possible ends. But

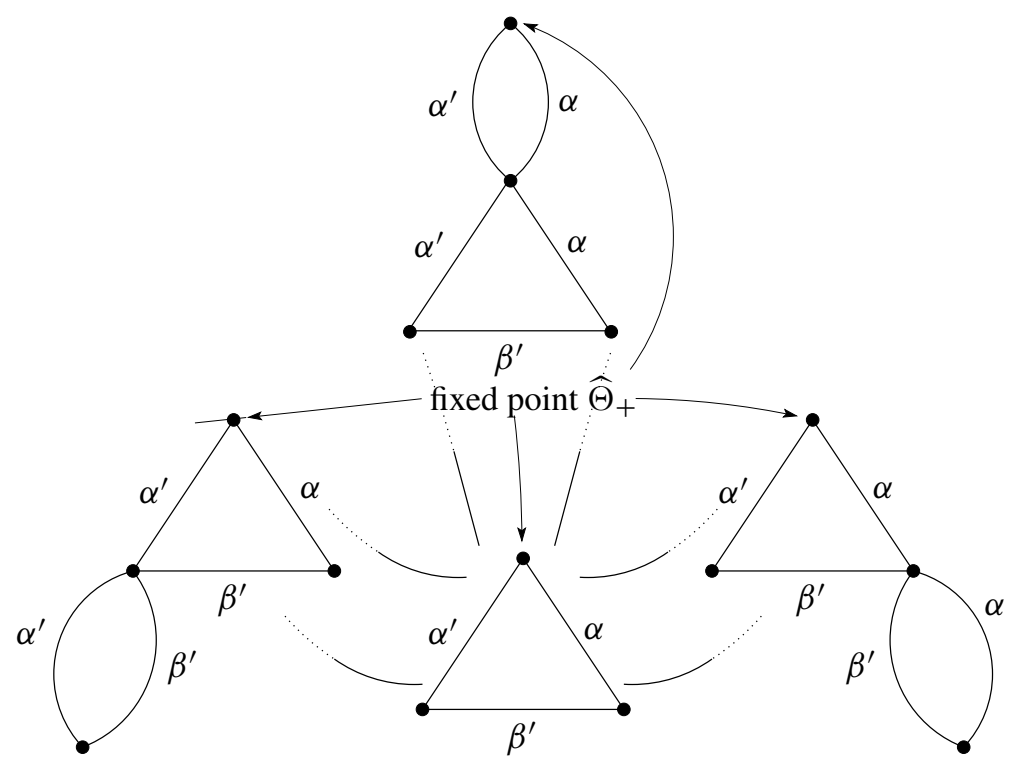

Figure 13: The moduli space has three possible ends. But only two of them count non-trivially, since $\widehat{\partial} \widehat{\Theta}^{+}=0$

observe that the top end (see Figure 13) corresponds to $\Gamma\left(x \otimes \widehat{\partial} \widehat{\Theta}^{+}\right)$, which vanishes since by definition $\widehat{\partial} \widehat{\Theta}^{+}=0$. Hence, for our situation there are just two possible types of ends to consider (the both at the bottom of Figure 13). Recall that breaking is the only phenomenon that appears here (see the proof of Proposition 4.4 or see Ozsváth and Szabó [23]). Proceeding as in the proof of Proposition 4.4, the commutativity of the third box follows. 
Proposition 5.2 Isotopies of the $\alpha$-circles induce isomorphisms on the homologies such that all squares commute. Isotopies of the $\beta$-curves that miss the points $w$ and $z$ induce isomorphisms such that all squares commute.

Proof We realize isotopies of the attaching circles by Hamiltonian isotopies. Hence, the induced map $\Phi$ on homology is defined by counting discs with dynamic boundary conditions in the $\alpha$-curves. The $\beta$-side remains untouched. Hence, by an analogous argument as in the proofs of Theorems 4.4 and 5.1 the map on homology splits into three components. The commutativity with $\Gamma_{1}$ and $\Gamma_{2}$ is then obviously true, and the only thing to show is the commutativity with the connecting homomorphism $\partial_{*}$ and $\partial_{*}^{\prime}$. But this again can be done by counting appropriate ends of moduli spaces or by looking into the chain map equation of $\Phi$ with respect to the representation of $\hat{\partial}^{\delta}$. $\square$

Consider the following situation: Let $(\Sigma, \alpha, \beta, z)$ be a $\delta$-suitable Heegaard diagram. With the discussion in Section 4.1 we obtain a long exact sequence

$$
\ldots \stackrel{\partial_{*}}{\longrightarrow} \widehat{\operatorname{HFK}}(\Sigma, \alpha, \beta, z, w) \stackrel{\Gamma_{1}}{\longrightarrow} \widehat{\mathrm{HF}}\left(\Sigma, \alpha, \beta^{\prime}, z\right) \stackrel{\Gamma_{2}}{\longrightarrow} \widehat{\operatorname{HFK}}(\Sigma, \alpha, \delta, z, w) \stackrel{\partial_{*}}{\longrightarrow} \ldots
$$

where we define the attaching circles

$$
\begin{aligned}
\beta^{\prime} & =\left\{\beta_{1}^{\prime}, \beta_{2}, \ldots, \beta_{g}\right\} \\
\delta & =\left\{\delta, \beta_{2}, \ldots, \beta_{g}\right\}
\end{aligned}
$$

as it was done in Section 4.1. Define $\beta^{\prime \prime}$ by performing a handle slide among the $\beta_{i}$, $i \geq 2$, or by a handle slide of $\beta_{1}^{\prime}$ over $\beta_{i}$. Perform the same operation on the set of attaching circles $\beta$ to obtain $\widetilde{\beta}$. Finally, take an isotopic push-off of $\delta, \delta^{\prime}$ say, that intersects $\delta$ in a cancelling pair of intersection points. Do the same with the $\beta_{i}, i \geq 2$, to get $\beta_{i}^{\prime}, i \geq 2$. In this way we define another set of attaching circles $\delta^{\prime}$ which is given by

$$
\delta^{\prime}=\left\{\delta^{\prime}, \beta_{2}^{\prime}, \ldots, \beta_{g}^{\prime}\right\} .
$$

Using these data we have the following result.

Proposition 5.3 In the present situation, denote by

$$
\begin{aligned}
\Gamma_{\alpha ; \beta, \widetilde{\beta}}^{w}: & \widehat{\mathrm{CFK}}(\Sigma, \alpha, \beta, z, w) \longrightarrow \widehat{\mathrm{CFK}}(\Sigma, \alpha, \tilde{\beta}, z, w) \\
\Gamma_{\alpha ; \delta, \delta^{\prime}}^{w}: & \widehat{\mathrm{CFK}}(\Sigma, \alpha, \delta, z, w) \longrightarrow \widehat{\mathrm{CFK}}\left(\Sigma, \alpha, \delta^{\prime}, z, w\right) \\
\Gamma_{\alpha ; \beta^{\prime}, \beta^{\prime \prime}} & : \widehat{\mathrm{CF}}\left(\Sigma, \alpha, \beta^{\prime}, z\right) \longrightarrow \widehat{\mathrm{CF}}\left(\Sigma, \alpha, \beta^{\prime \prime}, z\right)
\end{aligned}
$$


the induced maps between the associated chain complexes. These maps induce a commutative diagram with exact rows

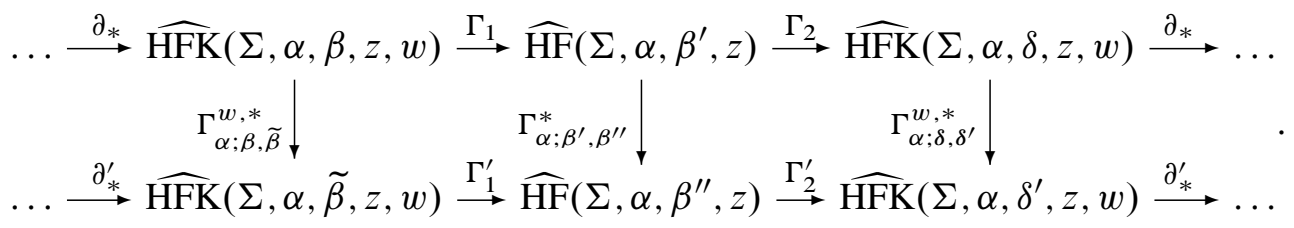

Before going in medias res, we would like to explain our strategy. The idea behind all main proofs concerning the exact sequences was to show that certain holomorphic discs cannot exist. Up to this point we always used the base points $w$ and $z$ in the sense that we tried to see what implications can be made from the conditions $n_{z}=n_{w}=0$. In addition, keeping in mind that holomorphic maps between manifolds of the same dimension are orientation preserving, we were able to prove everything we needed. Here, however, it is not so easy. First we would like to to see that the map $\Gamma_{\alpha ; \beta^{\prime}, \beta^{\prime \prime}}$ can be written as

$$
\Gamma_{\alpha ; \beta^{\prime}, \beta^{\prime \prime}}=\left(\begin{array}{cc}
\Gamma_{1} & \bar{\Gamma} \\
0 & \Gamma_{2}
\end{array}\right)
$$

This means we would like to show that there are no triangles connecting $\alpha \beta$-intersections of $\mathbb{T}_{\alpha} \cap \mathbb{T}_{\beta}$ with $\alpha \delta^{\prime}$-intersections of $\mathbb{T}_{\alpha} \cap \mathbb{T}_{\beta^{\prime \prime}}$ (see Figure 14). This part is very similar to the proofs already given. We could try to continue in the same spirit and identify moduli spaces as we did before, but this is quite messy in this situation. The reason is that we are counting triangles, and being forced to make an intermediate stop at the point $\widehat{\Theta}$, we are able to switch our direction there. So, comparing the boundary conditions given in the three triple diagrams is not very convenient. Unfortunately we were not able to avoid these inconveniences completely, but could minimize them. After proving the splitting, we stick to $\Gamma_{\alpha ; \beta^{\prime}, \beta^{\prime \prime}}$ and show that the maps $\Gamma_{1}, \Gamma_{2}, \bar{\Gamma}$ are chain maps and that all boxes in the diagram commute. This is realized by counting ends of appropriate moduli spaces of holomorphic triangles and squares. Finally, to minimize the messy task of comparing triangles in three diagrams, we just stick to $\Gamma_{1}$ and show that this map essentially equals $\Gamma_{\alpha ; \beta, \tilde{\beta}}^{w}$ on the chain level. The 5-Lemma then ends the proof.

Proof First observe that $\beta_{1}^{\prime}$ and $\beta_{1}^{\prime \prime}$ meet in two pairs of cancelling intersection points. Thus

$$
\begin{aligned}
\Gamma_{\alpha ; \beta^{\prime}, \beta^{\prime \prime}} & =\widehat{f}_{\alpha \beta^{\prime} \beta^{\prime \prime}}(\cdot \otimes \widehat{\Theta}) \\
& =\widehat{f}_{\alpha \beta^{\prime} \beta^{\prime \prime}}\left(\cdot \otimes\left\{a_{1}, \theta_{2}, \ldots, \theta_{g}\right\}\right)+\widehat{f}_{\alpha \beta^{\prime} \beta^{\prime \prime}}\left(\cdot \otimes\left\{a_{2}, \theta_{2}, \ldots, \theta_{g}\right\}\right) .
\end{aligned}
$$




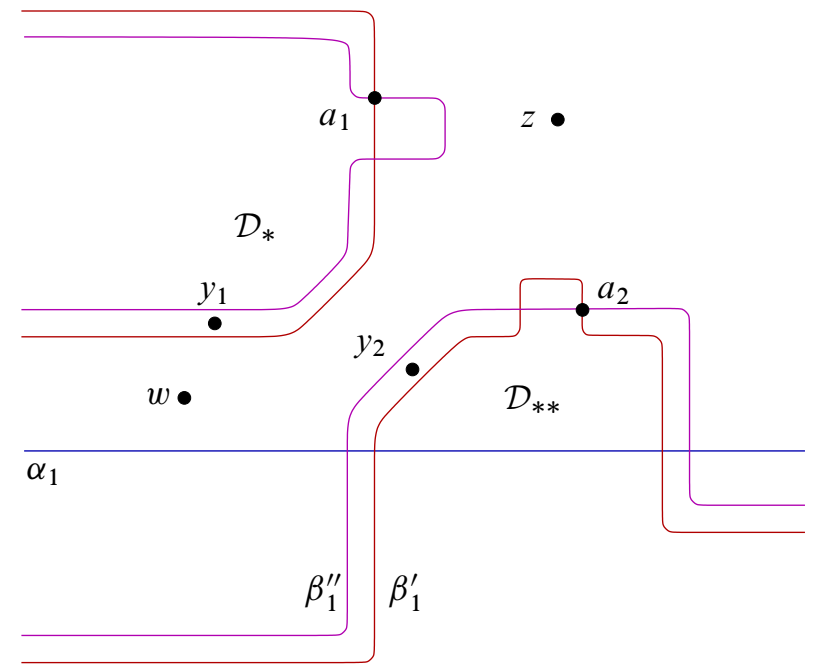

Figure 14: The important part of the Heegaard diagram after handle slide

So, we are looking for triangles with intermediate intersection $\left\{a_{1}, \theta_{2}, \ldots, \theta_{g}\right\}$ and triangles with intermediate intersection $\left\{a_{2}, \theta_{2}, \ldots, \theta_{g}\right\}$.

Step 1: Splitting Let $x \in \mathbb{T}_{\alpha} \cap \mathbb{T}_{\beta}$ and $y \in \mathbb{T}_{\alpha} \cap \mathbb{T}_{\widetilde{\beta}}$ be fixed. Let

$$
\left.\widehat{f}_{\alpha \beta^{\prime} \beta^{\prime \prime}}\left(x \otimes\left\{a_{1}, \theta_{2}, \ldots, \theta_{g}\right\}\right)\right|_{y}
$$

be the coefficient of $\widehat{f}_{\alpha \beta^{\prime} \beta^{\prime \prime}}\left(x \cdot \otimes\left\{a_{1}, \theta_{2}, \ldots, \theta_{g}\right\}\right)$ at the generator $y$. Suppose were is a triangle starting at $x$ and going to $y$ along the $\alpha$-boundary and then running to $a_{1}$ along its $\beta^{\prime}$-boundary. From that point we have to go back to $x$ again, following the red curve pictured in Figure 14. At $a_{1}$ we have two choices: we go upwards along the red curve, or we go downwards. Observe that going upwards, this would lead us to entering the $\mathcal{D}_{z}$-region at some point and force $n_{z}$ to be non-zero in contradiction to our assumptions. Going downwards, we again enter the $\mathcal{D}_{z}$-region and the boundary conditions force $n_{z}$ to be non-zero, again. Thus, there is no holomorphic triangle connecting $x$ with $y$ along $a_{1}$. Thus

$$
\left.\widehat{f}_{\alpha \beta^{\prime} \beta^{\prime \prime}}\left(x \otimes\left\{a_{1}, \theta_{2}, \ldots, \theta_{g}\right\}\right)\right|_{y}=0 .
$$

The next step is to compute

$$
\left.\widehat{f}_{\alpha \beta^{\prime} \beta^{\prime \prime}}\left(x \otimes\left\{a_{2}, \theta_{2}, \ldots, \theta_{g}\right\}\right)\right|_{y} .
$$

Suppose there were a triangle that contributes. Going along the boundary of that triangle we would start at $x$ and go to $y$ along the $\alpha$-boundary of the triangle and then try to 
go to $a_{2}$ following the pink curve in Figure 14. At some point we enter $\mathcal{D}_{z}$ forcing $n_{z}$ to be non-trivial. Hence, we have

$$
\left.\widehat{f}_{\alpha \beta^{\prime} \beta^{\prime \prime}}\left(x \otimes\left\{a_{2}, \theta_{2}, \ldots, \theta_{g}\right\}\right)\right|_{y}=0 .
$$

This shows that

$$
\Gamma_{\alpha ; \beta^{\prime}, \beta^{\prime \prime}}=\left(\begin{array}{cc}
\Gamma_{1} & \bar{\Gamma} \\
0 & \Gamma_{2}
\end{array}\right)
$$

Step 2: $\Gamma_{1}=\Gamma_{\alpha ; \beta, \tilde{\beta}}^{w}$ First of all it is easy to see that holomorphic triangles, contributing in $\Gamma_{\alpha ; \beta, \widetilde{\beta}}^{w}$, fulfil the property that $n_{y_{1}}=0$. Hence, together with $n_{w}=n_{z}=0$ the triangles have to stay away from the regions surrounding $\beta \cap \delta$. Hence, we have

$$
\Gamma_{1}=\Gamma_{\alpha ; \beta, \tilde{\beta}}^{w}+R .
$$

The map $R$ counts all holomorphic triangles not contributing to $\Gamma_{\alpha ; \beta, \tilde{\beta}}^{w}$. Conversely, all holomorphic discs contributing to $\Gamma_{1}$ should be shown to fulfil $n_{*}=n_{* *}=n_{y_{1}}=$ $n_{y_{2}}=0$. In this case $R=0$ and both maps coincide on the chain level. Look at Figure 15: The situation for the $\alpha \beta \widetilde{\beta}$-diagram is pictured.

(1) Observe that there is exactly one holomorphic triangle with $n_{* *} \neq 0$. This triangle contributes to $\bar{\Gamma}$.

(2) There is no holomorphic triangle contributing to $\Gamma_{1}$ with $n_{*} \neq 0$.

(3) In a similar vein observe that these triangles in addition have trivial intersection with $y_{1}$ and $y_{2}$.

Thus, we see that $R=0$.

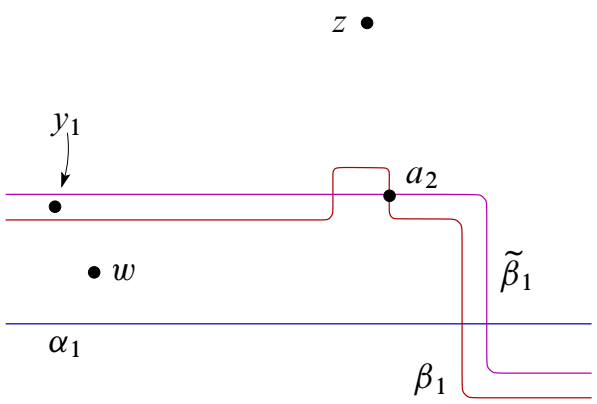

Figure 15: What happens

Step 3: Chain map properties and commutativity Given points $x \in \mathbb{T}_{\alpha} \cap \mathbb{T}_{\delta}$ and $y \in \mathbb{T}_{\alpha} \cap \mathbb{T}_{\tilde{\beta}}$, look at the moduli space of holomorphic triangles connecting $x$ with $y$, 
with Maslov index 1. There are, a priori, eight ends from which we just write down four. The four ends missing in Figure 16 are those contributing to $\Gamma(\cdot \otimes \partial \widehat{\Theta})$, which vanishes since $\partial \widehat{\Theta}=0$. We know that $\Gamma_{\alpha ; \beta^{\prime}, \beta^{\prime \prime}}$ is a chain map, that is,

$$
\begin{aligned}
0= & \partial \circ \Gamma_{\alpha ; \beta^{\prime}, \beta^{\prime \prime}}+\Gamma_{\alpha ; \beta^{\prime}, \beta^{\prime \prime}} \circ \partial \\
= & \partial_{\alpha \widetilde{\beta}^{w}}^{w} \circ \Gamma_{1}+\Gamma_{1} \circ \partial_{\alpha \beta}^{w} \\
& +\partial_{\alpha \widetilde{\beta}^{w}}^{w} \circ \bar{\Gamma}+f^{\prime} \circ \Gamma_{2}+\Gamma_{1} \circ f+\bar{\Gamma} \circ \partial_{\alpha \delta}^{w} \\
& +\partial_{\alpha \delta^{\prime}}^{w} \circ \Gamma_{2}+\Gamma_{2} \circ \partial_{\alpha \delta}^{w} .
\end{aligned}
$$

The first two terms vanish since we identified $\Gamma_{1}$ with $\Gamma_{\alpha ; \beta, \widetilde{\beta}}^{w}$, which is a $\left(\partial_{\alpha \beta}^{w}, \partial_{\alpha \widetilde{\beta}}^{w}\right)-$ chain map. The next four terms vanish since these correspond to the ends illustrated in Figure 16. Finally, since the whole equation is zero. the last two terms cancel each other. Thus, $\Gamma_{2}$ is a chain map as desired. By construction, two of three boxes in the diagram commute. We have to see that on the level of homology

$$
\Gamma_{1} \circ f=f^{\prime} \circ \Gamma_{2} .
$$

Recall we showed that on the chain level

$$
\partial_{\alpha \tilde{\beta}}^{w_{\tilde{S}}} \bar{\Gamma}+f^{\prime} \circ \Gamma_{2}+\Gamma_{1} \circ f+\bar{\Gamma} \circ \partial_{\alpha \delta}^{w}=0 .
$$

Hence, $\bar{\Gamma}$ is a chain homotopy between $\Gamma_{1} \circ f$ and $f^{\prime} \circ \Gamma_{2}$.

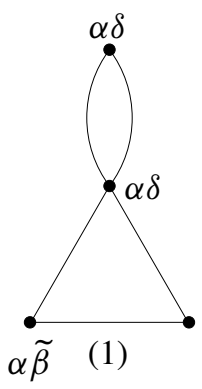

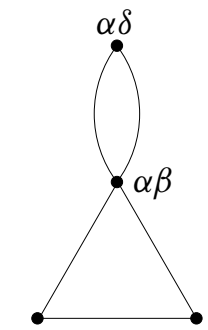

(2)

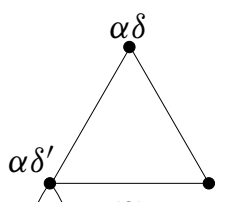

(3)

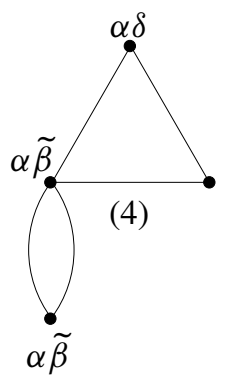

Figure 16: The ends of the moduli space providing commutativity

In [15], Lisca, Ozsváth, Stipsicz and Szabó give an alternative proof for the independence of the contact element of the choice of cut system. We are especially interested in the technique they used to prove [15, Proposition 3.3]. Recall, that given an open book $(P, \phi)$, a positive Giroux stabilization of $(P, \phi)$ is the open book $\left(P \cup h^{1}, \phi \circ D_{\gamma}^{+}\right)$ 


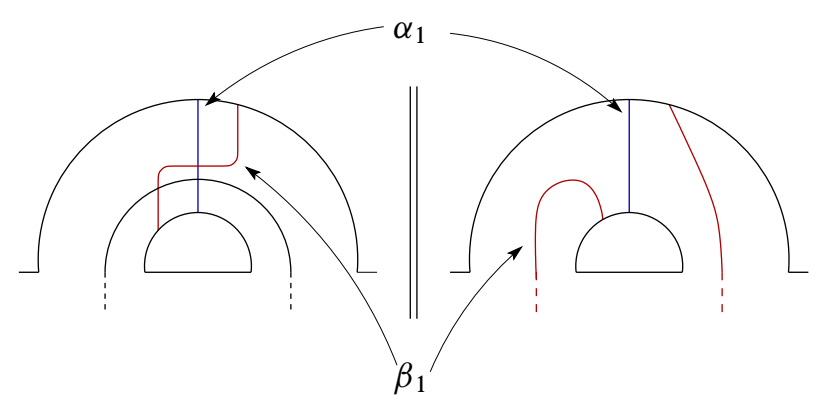

Figure 17: Illustration of what happens while Giroux stabilizing

where $\gamma$ is a closed curve in $P \cup h^{1}$ that intersects the co-core of $h^{1}$ once, transversely. Fixing a homologically essential, simple closed curve $\delta$ in $P$ we call the Giroux stabilization $\delta$-elementary if, after a suitable isotopy, $\delta$ intersects $\gamma$ transversely in at most one point (see [15, Definition 2.5]). Their invariance proof relies on the fact that, given a positive Giroux stabilization, one can choose a cut system $a_{1}, \ldots, a_{n}$ of $(P, \phi)$ such that the curve $\gamma$ does not intersect any of the $a_{i}$. Observe that, given such a cut system for $(P, \phi)$ and defining $a_{n+1}$ to be the co-core of the handle $h^{1}$, then $a_{1}, \ldots, a_{n+1}$ is a cut system for the Giroux stabilized open book. Furthermore, observe that for $i \leq n$

$$
\phi \circ D_{\gamma}^{+}\left(a_{i}\right)=\phi\left(a_{i}\right) \text {. }
$$

Figure 17 illustrates how $\phi \circ D_{\gamma}^{+}\left(\alpha_{n+1}\right)$ looks like. Thus, all intersections between $\alpha_{i}$ and $\beta_{j}$ for $i, j \leq n$ remain unchanged, where $\alpha_{n+1}$ intersects only $\beta_{n+1}$ once, transversely. Furthermore, $D_{\gamma}^{+}\left(a_{n+1}\right)$ is disjoint from all $a_{i}, i \leq n$. And, hence, $\beta_{n+1}$ is disjoint from all $\alpha_{i}, i \leq n$. Thus, the induced Heegaard diagram looks like a stabilized Heegaard diagram induced by the open book $(P, \phi)$ with cut system $a_{1}, \ldots, a_{n}$. Denote by $q$ the unique intersection point of $\alpha_{n+1}$ and $\beta_{n+1}$. Then the map

$$
\Phi: \widehat{\mathrm{CF}}\left(P, \phi,\left\{a_{1}, \ldots, a_{n}\right\}\right) \longrightarrow \widehat{\mathrm{CF}}\left(P \cup h^{1}, D_{\gamma}^{+} \circ \phi,\left\{a_{1}, \ldots, a_{n+1}\right\}\right),
$$

given by sending a generator $x$ of $\widehat{\mathrm{CF}}\left(P, \phi,\left\{a_{1}, \ldots, a_{n}\right\}\right)$ to $\Phi(x)=(x, q)$, is clearly an isomorphism of chain complexes preserving the contact element.

We will, however, focus our attention on a special version of positive Giroux stabilization. Recall, that we call $\left(\Sigma \# T^{2}, \alpha^{\prime}, \beta^{\prime}\right)$ a stabilization of the Heegaard diagram $(\Sigma, \alpha, \beta)$ where we define $\alpha^{\prime}=\alpha \cup\{\mu\}$ and $\beta^{\prime}=\beta \cup\{\lambda\}$ with $\mu$ a meridian and $\lambda$ a longitude of $T^{2}$. 


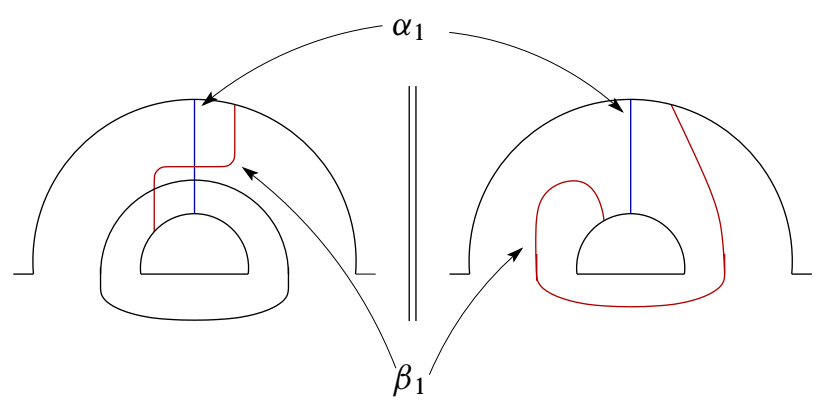

Figure 18: The choice of $\gamma$ for a topological stabilization

Definition 5.4 Let $(P, \phi)$ be an open book decomposition and let $\left(P^{\prime}, \phi \circ D_{\gamma}^{+}\right)$be a positive Giroux stabilization. We say that the Giroux stabilization represents a topological stabilization if there is a cut system $\left\{a_{1}, \ldots, a_{n}, a_{n+1}\right\}$ of $P^{\prime}$ with the following properties:

(1) The set $\left\{a_{1}, \ldots, a_{n}\right\}$ is a cut system for $P$.

(2) Denote by $(\Sigma, \alpha, \beta)$ the Heegaard diagram induced by $\left(P, \phi,\left\{a_{1}, \ldots, a_{n}\right\}\right)$ and let $\left(\Sigma^{\prime}, \alpha^{\prime}, \beta^{\prime}\right)$ be that induced by $\left(P^{\prime}, \phi \circ D_{\gamma}^{+},\left\{a_{1}, \ldots, a_{n+1}\right\}\right)$. The diagram $\left(\Sigma^{\prime}, \alpha^{\prime}, \beta^{\prime}\right)$ is a stabilization of $(\Sigma, \alpha, \beta)$ up to isotopy of the attaching circles.

Look into Figure 18. In this picture we present how to choose $\gamma$ such that the positive Giroux stabilization represents a topological stabilization. Indeed, the following lemma holds.

Lemma 5.5 Let $(P, \phi)$ be an open book decomposition and let $\left(P^{\prime}, \phi \circ D_{\gamma}^{+}\right)$be a positive Giroux stabilization. The Giroux stabilization represents a topological stabilization up to isotopy of the attaching circles if and only if $\gamma$ is isotopic to the black curve pictured in Figure 18.

Proof Given an open book decomposition $(P, \phi)$ and a positive Giroux stabilization $\left(P^{\prime}, \phi \circ D_{\gamma}^{+}\right)$with $\gamma$ like indicated in Figure 18 , this stabilization clearly represents a topological stabilization up to isotopy: Recall that $P^{\prime}=P \cup h^{1}$. Choose a cut system $\left\{a_{1}, \ldots, a_{n}\right\}$ of $P$ such that $\partial a_{i}, i=1, \ldots, n$, is disjoint from the region where the handle $h^{1}$ is attached on. Define $a_{n+1}$ as the co-core of the handle $h^{1}$. Picturing the resulting Heegaard diagrams we see that the positive Giroux stabilization represents a topological stabilization up to isotopy.

Conversely, suppose we are given a Giroux stabilization representing a topological stabilization up to isotopy, then we have to show that $\gamma$ is isotopic to the black curve, 
$\gamma_{s}$ say, indicated in Figure 18. First note that the handle is attached on one boundary component of $P$. If $h^{1}$ connects two different boundary components of $P$, the genus of the resulting Heegaard surface would increase by 2 . By assumption there is a cut system $\left\{a_{1}, \ldots, a_{n+1}\right\}$ for $P^{\prime}$ fulfilling properties (1) and (2), given in Definition 5.4. As in Definition 5.4, denote by $(\Sigma, \alpha, \beta)$ and $\left(\Sigma^{\prime}, \alpha^{\prime}, \beta^{\prime}\right)$ the respective Heegaard diagrams. By assumption, $\Sigma^{\prime}=\Sigma \# T^{2}$ and, after applying suitable isotopies, $\alpha_{i}=\alpha_{i}^{\prime}$ and $\beta_{i}=\beta_{i}^{\prime}$ for all $i=1, \ldots, n$. We have

$$
\begin{aligned}
& \alpha_{n+1}^{\prime}=a_{n+1} \cup \overline{a_{n+1}} \\
& \beta_{n+1}^{\prime} \sim a_{n+1} \cup \overline{\phi \circ D_{\gamma}^{+}\left(a_{n+1}\right)}
\end{aligned}
$$

with

$$
\begin{aligned}
\alpha_{n+1}^{\prime} & \sim \mu_{T^{2}} \\
\beta_{n+1}^{\prime} & \sim \lambda_{T^{2}} .
\end{aligned}
$$

By (5-2), we see that $a_{n+1}$ is isotopic to the co-core of $h^{1}$. This can be read off from Figure 19.

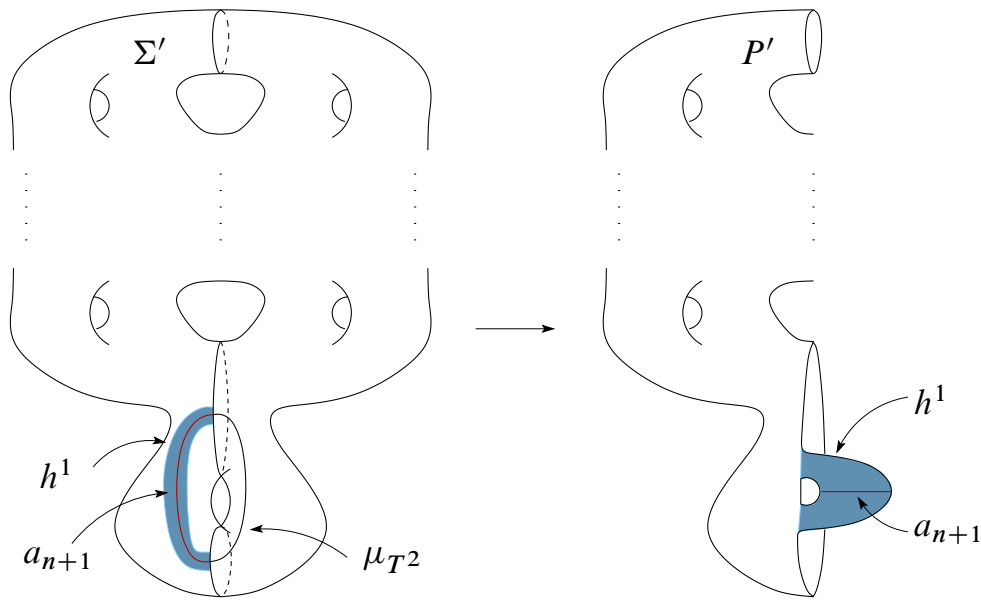

Figure 19: The left portion pictures $\Sigma^{\prime}$ and the right portion the page $P^{\prime}$ and how it is obtained from $P$

Hence, we have

$$
a_{n+1} \cup \overline{\phi \circ D_{\gamma}^{+}\left(a_{n+1}\right)}=\beta_{n+1} \sim \lambda_{T^{2}} \sim a_{n+1} \cup \overline{\phi \circ D_{\gamma_{s}}^{+}\left(a_{n+1}\right)} .
$$

So, $\phi \circ D_{\gamma}^{+}\left(a_{n+1}\right)$ is isotopic to $\phi \circ D_{\gamma_{s}}^{+}\left(a_{n+1}\right)$, which is equivalent to saying that $D_{\gamma}\left(a_{n+1}\right)$ is isotopic to $D_{\gamma_{s}}\left(a_{n+1}\right)$. But this finally implies that $\gamma$ is isotopic to $\gamma_{s}$. 
Proposition 5.6 Let $(P, \phi)$ be an open book decomposition of $Y$ and $\left(P^{\prime}, \phi \circ D_{\gamma}^{+}\right)$ a positive $\delta$-elementary Giroux stabilization representing a topological stabilization (see Definition 5.4 and look at Figure 18). Then there are isomorphisms $\phi_{1}, \phi_{2}$ and $\phi_{3}$ on homology such that the following diagram commutes:

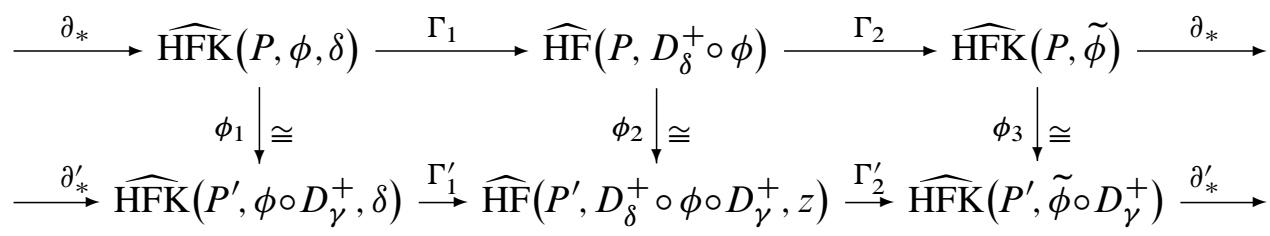

Remark 3 General positive Giroux stabilizations do not preserve the exact sequence. The reason is that in the general situation $\gamma \cap P$ and $\phi^{-1}(\delta)$ might intersect and cannot be separated. In the topological situation, however, the special choice of $\gamma$ makes it possible to separate $\gamma \cap P$ from $\phi^{-1}(\delta)$.

Proof Denote by $\gamma_{1}$ the part of $\gamma$ that runs through $P$. Since we are just doing a topological stabilization, we can attach the handle $h^{1}$ in such a way that $\gamma_{1}$ and $\phi^{-1}(\delta)$ are disjoint. Just choose $\gamma$ like indicated in Figure 18. Even if $\phi^{-1}(\delta)$ intersects $\gamma_{1}$, we can separate them with help of a small isotopy. By choosing a cut system $\left\{a_{1}, \ldots, a_{n}\right\}$ for $(P, \phi)$ appropriately, we can extend this cut system to a cut system for the stabilized open book by choosing $a_{n+1}$ like indicated in Figure 18. For all Heegaard diagrams in the following, we will use this cut system. Since $\phi^{-1}(\delta)$ and $\gamma$ are disjoint, the associated Heegaard diagram of $\left(P^{\prime}, D_{\delta}^{+} \circ \phi \circ D_{\gamma}^{+}\right)$will look like a stabilization of the Heegaard diagram induced by the open book $\left(P, D_{\delta}^{+} \circ \phi\right)$. The same holds for $\left(-P^{\prime}, \tilde{\phi}\right)$ and $\left(-P^{\prime}, \tilde{\phi} \circ D_{\gamma}^{+}\right)$. Using the isomorphism induced by stabilizations as discussed above we can define $\phi_{1}, \phi_{2}$ and $\phi_{3}$ as indicated in Proposition 5.6. These maps are all isomorphisms and obviously commute on the chain level.

Theorem 5.7 The map $\Gamma_{1}$ is topological, that is, it just depends on the cobordism induced by the surgery.

Proof The cobordism induced by the Dehn twist depends only on the 3-manifold $Y$ and the framed knot type $K$ which the curve $\delta$, together with its page framing, represents inside $Y$. This pair, on the other hand, is described by an open book decomposition adapted to $\delta$ and a $\delta$-adapted cut system. These data determine a Heegaard diagram subordinate to the pair $(Y, K)$ (see Section 2.2.1). Given another adapted open book together with an adapted cut system, the associated Heegaard diagram is equivalent to the first after a sequence of moves which are described in Lemma 2.3. All of these moves are recovered via Proposition 5.1, Proposition 5.2, 
Proposition 5.3 and Proposition 5.6. Of course, after some point, we might leave the class of Heegaard diagrams induced by open books. But the propositions cited do not use this open book structure as discussed at the beginning of the section.

\section{Implications to contact geometry}

In this section we will focus our attention on contact manifolds $(Y, \xi)$. Let $(P, \phi)$ be an open book decomposition that is adapted to the contact structure $\xi$ (see Section 2.4). Recall that the contact element and the invariant defined by Lisca, Ozsváth, Stipsicz and Szabó [15] sit in the Heegaard Floer cohomology (see Section 2.4). Because of the well-known equivalence

$$
\widehat{\mathrm{HF}}^{*}(Y)=\widehat{\mathrm{HF}}_{*}(-Y)
$$

we will be interested in the behavior of $-Y$ rather than $Y$. Recall from Section 2.4 that we have two choices to extract the Heegaard Floer homology of $-Y$ from data given by a Heegaard diagram of $Y$. We can either switch the orientation of the Heegaard surface or switch the boundary conditions.

Let $L \subset Y$ be a Legendrian knot and denote by $Y_{L}^{+}$the manifold obtained by doing a $(+1)$-contact surgery along $L$. There is an open book decomposition $(P, \phi)$ adapted to $\xi$ such that $L$ sits on the page $P \times\{1 / 2\}$ of the open book and the page framing coincides with the contact framing. A $(+1)$-contact surgery acts on the open book like a negative Dehn twist along $L$, that is, $\left(P, \phi \circ D_{L}^{-, P}\right)$ is an adapted open book decomposition of $\left(Y_{L}^{+}, \xi_{L}^{+}\right)$where $D_{L}^{-, P}$ denotes a negative Dehn twist along $L$ with respect to the orientation of $P$. Observe that $L$ sits on the wrong page for our construction of the exact sequence. Fortunately, the identity

$$
\phi \circ D_{L}^{-, P}=D_{\phi(L)}^{-, P} \circ \phi
$$

holds. Thus, a surgery along $L$ can be interpreted as a left-hand composition of the monodromy with a Dehn twist. In addition $\left(P, D_{\phi(L)}^{-, P} \circ \phi\right)$ is an adapted open book decomposition of $\left(Y_{L}^{+}, \xi_{L}^{+}\right)$. To see the effect on the Heegaard Floer cohomology, we have to change the surface orientation. We see that

$$
-Y_{L}^{+}=\left(-P, D_{\phi(L)}^{-, P} \circ \phi\right)=\left(-P, D_{\phi(L)}^{+,-P} \circ \phi\right) .
$$

One very important ingredient for our construction is the fact that we may choose an $L$-adapted Heegaard diagram where $L$ sits on $P \times\{1 / 2\}$. Because of the identity (6-1) we need a Heegaard diagram with attaching circles adapted to $\phi(L)$ in the following sense: the curve $\phi(L)$ intersects $\beta_{1}$ once, transversely and is disjoint from all other $\beta$-circles. This condition is satisfied for $L$-adapted Heegaard diagrams since 
$\phi\left(a_{i}\right)=b_{i}$. This means we are able to simultaneously match all conditions for setting up the exact sequence and seeing the invariant $\widehat{\mathcal{L}}(L)$. Recall that the sequence requires the point $w$ defining $L$ to be in a specific domain of the Heegaard diagram. This positioning of $w$ induces an orientation on $L$. On the other hand, a fixed orientation of $L$ determines where $w$ has to be placed. These two orientations, the one coming from the sequence and the one from the knot $L$ itself, have to be observed carefully. We have to see whether every possible choice of orientation of $L$ induces a positioning of $w$ inside the Heegaard diagram that is compatible with the requirements coming from the exact sequence.

Theorem 6.1 Let $(Y, \xi)$ be a contact manifold and $L \subset Y$ an oriented Legendrian knot.

(i) Let $W$ be the cobordism induced by $(+1)$-contact surgery along $L$. Then the cobordism $-W$ induces a map

$$
\Gamma_{-W}: \widehat{\mathrm{HFK}}(-Y, L) \longrightarrow \widehat{\mathrm{HF}}\left(-Y_{L}^{+}\right),
$$

such that $\Gamma_{-W}(\widehat{\mathcal{L}}(L))=c\left(Y_{L}^{+}, \xi_{L}^{+}\right)$.

(ii) If $L$ carries a specific orientation and $W$ denotes the cobordism induced by a $(-1)$-contact surgery along $L$. Then the cobordism $-W$ induces a map

$$
\Gamma_{-W}: \widehat{\mathrm{HF}}\left(-Y_{L}^{-}\right) \longrightarrow \widehat{\mathrm{HFK}}(-Y, L)
$$

such that $\Gamma_{-W}\left(c\left(Y_{L}^{-}, \xi_{L}^{-}\right)\right)=0$.

Proof Recall that

$$
\begin{aligned}
-Y_{L}^{+} & =\left(-P, D_{\phi(L)}^{+,-P} \circ \phi\right) \\
-Y_{L}^{-} & =\left(-P, D_{\phi(L)}^{-,-P} \circ \phi\right) .
\end{aligned}
$$

We choose a cut system which is $L$-adapted. This means that $L$ intersects $\alpha_{1}$ transversely, in a single point and is disjoint from the other $\alpha$-circles. Hence, $\phi(L)$ (sitting on the other side of the Heegaard surface) intersects $\beta_{1}$ in a single point and is disjoint from the other $\beta$-circles. We first try to prove the results concerning the $(+1)$-contact surgery. After possibly isotoping the knot $L$ slightly, we can achieve a neighborhood of $\phi(L) \cap \beta_{1}$ to look like the left or right part of Figure 20 .

In each part of the picture the knot $L$ and the point $w$ are placed in such a way that the Dehn twist associated to the $(+1)$-contact surgery connects the regions where the points $w$ and $z$ lie. Thus, each picture shows a situation in which we may apply the proof technique used for Proposition 4.4 (resp. Proposition 4.7). Observe that 

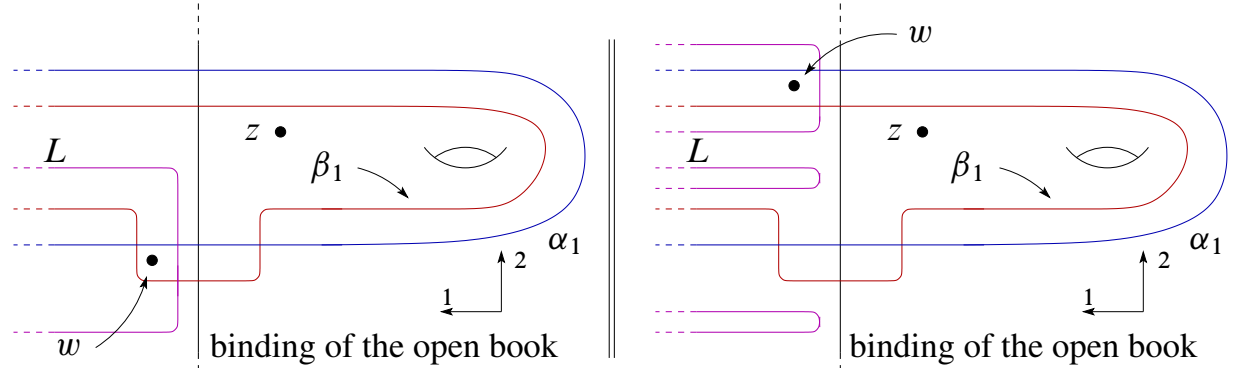

Figure 20: Setting things up for a contact $(+1)$-surgery

Figure 20 shows the situation for each orientation of $L$. Since we are doing a $(+1)-$ contact surgery, we perform a positive Dehn twist along $L$ with respect to the surface orientation given in Figure 20 (see Equality (6-2) and see the discussion at the beginning of this section). Thus, we are able to define a map

$$
\Gamma^{+}: \widehat{\operatorname{HFK}}(-Y, L) \longrightarrow \widehat{\mathrm{HF}}\left(-Y_{L}^{+}\right) \text {. }
$$

The situations in both pictures are designed to apply the proof technique of Proposition 4.4. The induced pair $(w, z)$ determines an orientation on $L$. To match the induced orientation with the one of the knot $L$ we either use the left or the right picture of Figure 20. By definition of $\Gamma^{+}$we see that

$$
\Gamma^{+}(\widehat{\mathcal{L}}(L))=c\left(Y_{L}^{+}, \xi_{L}^{+}\right) .
$$

To cover (-1)-contact surgeries, look at Figure 21.
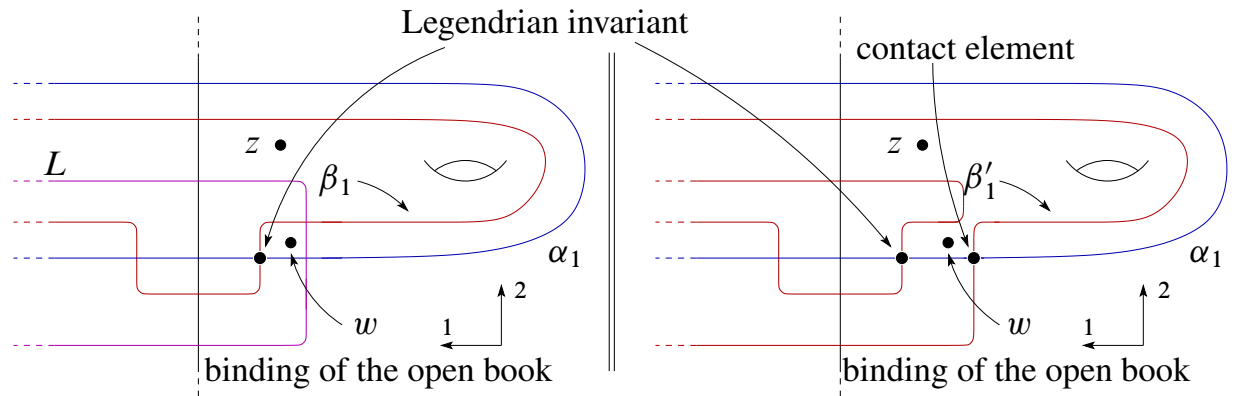

Figure 21: Setting things up for a contact $(-1)$-surgery

The same line of arguments as above applies to define a map

$$
\Gamma^{-}: \widehat{\mathrm{HF}}\left(-Y_{L}^{-}\right) \longrightarrow \widehat{\mathrm{HFK}}(-Y, L) \text {. }
$$


Again, recall that $w$ is placed in the Heegaard diagram in such a way that allows us to define the map $\Gamma^{-}$. The pair $(w, z)$ induces an orientation on $L$. The opposite orientation will be denoted by $o b$. What can be seen immediately from the picture is that the Dehn twist separates the contact element and the invariant $\widehat{\mathcal{L}}(L, \overline{o b})$ : The arguments show that we have the following exact sequence.

$0 \longrightarrow \widehat{\mathrm{CFK}}\left(Y_{0}(L), \mu\right) \longrightarrow \widehat{\mathrm{CF}}\left(-Y_{L}^{-}\right) \stackrel{\Gamma^{-}}{\longrightarrow} \widehat{\mathrm{CFK}}(-Y,(L, \overline{o b})) \longrightarrow 0$

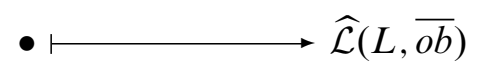

To speak in the language of the proof of Proposition 4.4: the element $c$ is an $\alpha \beta-$ intersection, whereas the element $\widehat{\mathcal{L}}(L, \overline{o b})$ is an $\alpha \delta$-intersection. By exactness, the contact element $c$ lies in the kernel of $\Gamma^{-}$.

Definition 6.2 The orientation $o b(P, \phi)$ from the last proof is called the open book orientation.

To prove Corollary 6.3 we have to recall that Honda, Kazez and Matić introduced in [12] an invariant $E H(L)$ of a Legendrian knot $L$ in the sutured Floer homology (see Juhasz [14]) of a contact manifold with boundary. To be more precise, given $L \subset(Y, \xi)$, they define an Legendrian isotopy invariant of $L$, called $E H(L)$, sitting in $\operatorname{SFH}(-Y \backslash v L, \Gamma)$ where $\Gamma$ are suitably chosen sutures. Furthermore, Stipsicz and Vertesi have shown in [26] that this invariant is equipped with a morphism $\mathrm{SFH}(-Y \backslash v L, \Gamma) \longrightarrow \widehat{\mathrm{HFK}}(-Y, L)$ that maps $E H(L)$ to $\widehat{\mathcal{L}}(L)$. Composing this morphism with the one coming from Theorem 6.1 we get the following result.

Corollary 6.3 There is a map

$$
\gamma: \operatorname{SFH}(-Y \backslash v L, \Gamma) \longrightarrow \widehat{\mathrm{HF}}\left(-Y_{L}^{+}\right)
$$

such that $\gamma(E H(L))=c\left(Y_{L}^{+}, \xi_{L}^{+}\right)$.

Corollary 6.4 Let $L$ be a Legendrian knot in a contact manifold $(Y, \xi)$. Then $E H(L)=0$ implies that $c\left(Y_{L}^{+}, \xi_{L}^{+}\right)=0$.

It is also possible to derive these corollaries using methods coming from [26].

Proposition 6.5 Let $L$ be a Legendrian knot in a contact manifold $(Y, \xi)$ carrying the open book orientation induced by an adapted open book $(P, \phi)$. Let $\left(P^{\prime}, \phi^{\prime}\right)$ be the once-stabilized open book that carries the Legendrian knot $S_{+}(L)$ (see Proposition 6.11). The open book orientation $o b\left(P^{\prime}, \phi^{\prime}\right)$ coincides with the orientation included by the stabilization. 
We will give a proof of Proposition 6.5 in the following paragraph.

\subsection{Stabilizations of Legendrian knots and open books}

6.1.1 Stabilizations as Legendrian band sums Recall that stabilization basically means to enter a zigzag into the front projection of a Legendrian knot. If we are not in the standard contact space, we perform this operation inside a Darboux chart. Which zigzag is regarded as a positive/negative stabilization depends on the knot orientation. Positivity/Negativity is fixed by the following equations

$$
\begin{aligned}
\operatorname{tb}\left(S_{ \pm}(L)\right) & =\operatorname{tb}(K)-1 \\
\operatorname{rot}\left(S_{ \pm}(L)\right) & =\operatorname{rot}(L) \pm 1 .
\end{aligned}
$$

This tells us that

$$
\overline{S_{+}(L)}=S_{-}(\bar{L})
$$

Given two Legendrian knots $L$ and $L^{\prime}$, we can form their Legendrian band sum $L \#_{L b} L^{\prime}$ in the following way: Pick a contact surgery representation of the contact manifold in such a way that the surgery link $\mathbb{L}$ stays away from $L \cup L^{\prime}$. In this way we can think of $L$ and $L^{\prime}$ as sitting in the standard contact space and, so, can perform the band sum. We denote by $L_{0}$ and $\bar{L}_{0}$ the oriented Legendrian shark with the orientations as indicated in Figure 22.
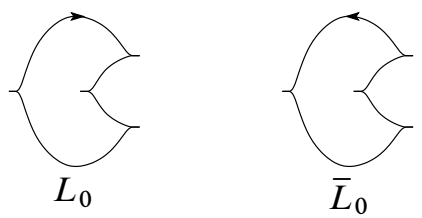

Figure 22: The oriented Legendrian shark and its inverse

Proposition 6.6 Given a Legendrian knot $L$, we can realize its stabilizations as Legendrian band sums, that is,

$$
\begin{aligned}
& S_{+}(L)=L \#_{L b} L_{0} \\
& S_{-}(L)=L \#_{L b} \bar{L}_{0},
\end{aligned}
$$

where $\#_{L b}$ denotes the Legendrian band-sum. 
Proof We prove the equality for positive stabilizations. The case of negative stabilizations is proved in a similar fashion. No matter what orientation the knot $L$ carries, we will find at least one right up-cusp or one right down-cusp. In case of a right down-cusp we perform a band-sum involving this right down-cusp on $L$ an the left up-cusp on $L_{0}$. In case we use a right up-cusp we perform the band-sum as indicated in the left part of Figure 23. In Figure 23 we indicate the Legendrian isotopy that illustrates that we have stabilized positively.

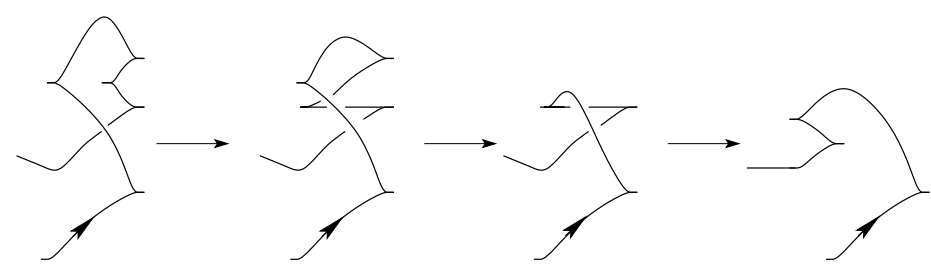

Figure 23: The Legendrian band-sum in case of a right up-cusp and a Legendrian isotopy

6.1.2 Open books and connected sums Suppose we are given open books $\left(P_{1}, \phi_{1}\right)$ and $\left(P_{2}, \phi_{2}\right)$ for manifolds $\left(Y_{1}, \xi_{1}\right)$ and $\left(Y_{2}, \xi_{2}\right)$. Let $B_{1}$ be the binding of $\left(P_{1}, \phi_{1}\right)$. Denote by $v B_{1}$ an equivariant tubular neighborhood of $B_{1}$. Fix a point $p$ on $B_{1}$ and embed a $3-$ ball $D^{3}$ such that it is centered at $p$. Furthermore, the ball should sit inside $\nu B_{1}$ such that the north and south pole of $D^{3}$ equal $B_{1} \cap \mathbb{S}^{2}$. Denote by $f_{1}: D^{3} \longrightarrow v B_{1} \subset Y_{1}$ the embedding. Embed $g: D^{3} \longrightarrow Y_{2}$ in the same fashion. Compose $g$ with a right-handed rotation $r$ that swaps the two hemispheres of $D^{3}$ to get another embedding $f_{2}=g \circ r$. Use these embeddings to perform the connected sum. By its definition, the gluing $f_{2} \circ f_{1}^{-1}$ preserves the open book structure. Note that the rotation is needed to make the pages of the open book glue together nicely with their given orientation. Moreover, we are able to explicitly describe the resulting open book. The new page $P$ equals $P_{1} \cup_{h^{1}} P_{2}$, where $h^{1}$ is a 1 -handle connecting $P_{1}$ and $P_{2}$ and the binding $B$ equals $B_{1} \# B_{2}$. To define the monodromy, first extend $\phi_{1}$ and $\phi_{2}$ as the identity along the handle and the complementary page. Then define $\phi$ as the composition $\phi_{2} \circ \phi_{1}=\phi_{1} \circ \phi_{2}$.

Lemma 6.7 The open book $(P, \phi)$ is an adapted open book for $\left(Y_{1} \# Y_{2}, \xi_{1} \# \xi_{2}\right)$.

Proof Observe that the given operation is a special case of the Murasugi sum. The lemma then follows from Etnyre [9]. 
Corollary 6.8 Let $(Y, \xi)$ and $\left(Y^{\prime}, \xi^{\prime}\right)$ be contact manifolds and $L \subset Y$ a Legendrian knot. Then we have

$$
\begin{array}{ccc}
\widehat{\mathrm{HFK}}\left(-Y \# Y^{\prime}, L\right) & \cong \widehat{\mathrm{HFK}}(-Y, L) \otimes \widehat{\mathrm{HF}}\left(-Y^{\prime}\right) \\
\widehat{\mathcal{L}}\left(Y \# Y^{\prime}, L\right) & =\widehat{\mathcal{L}}(Y, L) \otimes c\left(\xi^{\prime}\right)
\end{array}
$$

Proof Let $\left(P_{1}, \phi_{1}\right)$ be an open book decomposition adapted to the knot $L$ and the contact structure $\xi$. Denote by $\left(P_{2}, \phi_{2}\right)$ an open book for $\left(Y^{\prime}, \xi^{\prime}\right)$. We define an open book $(P, \phi)$ by using the open books for $Y$ and $Y^{\prime}$ as given above. Recall, that the page $P$ is given by joining the pages $P_{1}$ and $P_{2}$ with a 1 -handle $h^{1}$, that is,

$$
P=P_{1} \cup_{h^{1}} P_{2} \text {. }
$$

Denote by $f: \partial h^{1} \longrightarrow \partial P_{1} \sqcup \partial P_{2}$ the attaching map. Furthermore, let $\left\{a_{1}, \ldots, a_{n}\right\}$ be a cut system for $P_{1}$ and $\left\{a_{1}^{\prime}, \ldots, a_{m}^{\prime}\right\}$ a cut system for $P_{2}$. Choose isotopic push-offs $b_{i}$ of the $a_{i}$ so that $a_{i}$ and $b_{i}$ intersect each other in a pair $x_{i}^{+}, x_{i}^{-}$of intersection points. The push-offs are chosen like specified in Section 2.4 (see also Figure 1). Analogously, the curves $b_{j}^{\prime}, j=1, \ldots, m$, are defined; denote the points of intersection by $y_{j}^{+}, y_{j}^{-}$, $j=1, \ldots, m$. The names are attached to the intersection points in such a way that $\left\{x_{1}^{+}, \ldots, x_{n}^{+}\right\}$represents the class $\widehat{\mathcal{L}}(Y, L)$ and that $\left\{y_{1}^{+}, \ldots, y_{m}^{+}\right\}$represents $c\left(\xi^{\prime}\right)$. We additionally fix base points $z_{i} \in P_{i}, i=1,2$, and a third one, $w$ say, in $P_{1}$ determining the knot $L$. These choices induce Heegaard diagrams we denote by $\left(\Sigma_{i}, \alpha_{i}, \beta_{i}\right), i=1,2$. We require the chosen cut systems to fulfil the following two conditions:

(1) $\operatorname{Im}(f) \cap\left(\bigcup_{i=1}^{n} \partial a_{i} \cup \bigcup_{j=1}^{m} \partial a_{j}^{\prime}\right)=\varnothing$

(2) $\operatorname{Im}(f) \subset \partial \overline{\mathcal{D}_{z_{1}}} \cup \partial \overline{\mathcal{D}_{z_{2}}}$

As a consequence of these two conditions and the fact that by definition $\left.\phi\right|_{P_{i}}=\phi_{i}$, $i=1,2$ and $\left.\phi\right|_{h^{1}}=\mathrm{id}_{h^{1}}$ we see that

$$
\phi\left(a_{i}\right) \cap a_{j}^{\prime}=\varnothing \text { and } a_{i} \cap \phi\left(a_{j}^{\prime}\right)=\varnothing .
$$

The set $\left\{a_{1}, \ldots, a_{n}\right\} \cup\left\{a_{1}^{\prime}, \ldots, a_{m}^{\prime}\right\}$ is a cut system for the open book $(P, \phi)$. Denote by $(\Sigma, \alpha, \beta)$ the induced Heegaard diagram, then with (6-4), we see that

$$
\Sigma=\Sigma_{1} \# \Sigma_{2}, \alpha=\alpha_{1} \cup \alpha_{2}, \beta=\beta_{1} \cup \beta_{2}
$$

and the points $z_{i}, i=1,2$, lie in the regions unified by the connected sum tube. Choose a base point $z \in \Sigma$ lying in this unified region. Thus - with the same reasoning as in the proof of [22, Proposition 6.1] - we see that

$$
\widehat{\mathrm{HFK}}(-Y \# Y, L) \cong \widehat{\mathrm{HFK}}(-Y, L) \otimes \widehat{\mathrm{HF}}(-Y) \text {. }
$$


By construction, the intersection point $\left\{x_{1}^{+}, \ldots, x_{n}^{+}, y_{1}^{+}, \ldots, y_{m}^{+}\right\}$represents the class $\widehat{\mathcal{L}}\left(Y \# Y^{\prime}, L\right)$. But the isomorphism giving (6-5), $\varphi$ say, has that property that

$$
\left\{x_{1}^{+}, \ldots, x_{n}^{+}, y_{1}^{+}, \ldots, y_{m}^{+}\right\} \longmapsto\left\{x_{1}^{+}, \ldots, x_{n}^{+}\right\} \otimes\left\{y_{1}^{+}, \ldots, y_{m}^{+}\right\},
$$

that is, $\varphi\left(\widehat{\mathcal{L}}\left(-Y \# Y^{\prime}, L\right)\right)=\widehat{\mathcal{L}}(-Y, L) \otimes c\left(\xi^{\prime}\right)$.

Lemma 6.9 (Etnyre [9]) If $\gamma$ is a non-separating curve on a page of an open book $(P, \phi)$, we can isotope the open book slightly such that $\gamma$ is Legendrian and the contact framing agrees with the page framing.

This fact follows from the Legendrian realization principle. As a consequence, we get the following corollary.

Corollary 6.10 If the Legendrian knots $L_{i} \subset P_{i}$ sit on the ages, then, on the page $P$ of $(P, \phi)$, we will find a Legendrian knot $L$ with the following property: There is a naturally induced contactomorphism $\phi_{c}$ such that $\phi_{c}(L)$ equals $L_{1} \#_{L b} L_{2}$ after performing a right-handed twist along the Legendrian band. Indeed, we obtain $L$ by a band sum of $L_{1}$ and $L_{2}$ on the page $P$.

Proof Let $\left(P_{i}, \phi_{i}\right)$ be open books adapted to $\left(Y_{i}, \xi_{i}, L_{i}\right), i=1,2$. On $P_{i}$ there is a set of embedded, simple closed curves $c_{1}^{i}, \ldots, c_{n}^{i}$ whose associated Dehn twists generate the mapping class groups of $P_{i}$. The associated Dehn twists can be interpreted as contact surgeries along suitable Legendrian knots (see [15, Theorem 2.7]). Thus, using the open book decomposition we are able to find a (maybe very inefficient) contact surgery representation of $\left(Y_{i}, \xi_{i}\right)$ which is suitable for our purposes to perform the Legendrian band sum (see the beginning of this section). Moreover, we can think of $L_{1}$ to pass the binding $B_{1}$ of $P_{1}$ very closely at some point: this means that there is a point $p_{1}$ in the binding, and a Darboux ball $D_{1}$ around $p_{1}$, such that the curve intersects this Darboux ball. Suppose this is not the case, then we can isotope the Legendrian knot $L_{1}$, which sits on $P_{1}$, as a curve in $P_{1}$, to pass the binding closely (as described above). The isotopy is not necessarily a Legendrian isotopy. However, by [15, Theorem 2.7], we know that the isotoped curve determines a uniquely defined Legendrian knot, which is Legendrian isotopic to $L_{1}$. With a slight isotopy of the open book, we can think of this new knot as sitting on $P_{1}$. By abuse of notation, we call the new knot $L_{1}$. After possibly isotoping the open book we can think of $\mathbb{L}_{1}$ as sitting in the complement of $D_{1}$. We obtain a situation like indicated in the top row of Figure 24. Since we have the identification $\left(Y_{1}, \xi_{1}\right) \cong\left(\mathbb{S}^{3}\left(\mathbb{L}_{1}\right), \xi_{\mathbb{L}_{1}}\right)$, the ball $D_{1}$ can be thought of as sitting in $\mathbb{S}^{3}$. The complement of $D_{1}$ in $\mathbb{S}^{3}$ is again a ball we denote by $\widetilde{D}_{1}$. We may make similar arrangements for $L_{2}$ : however, we would like $L_{2}$ and 

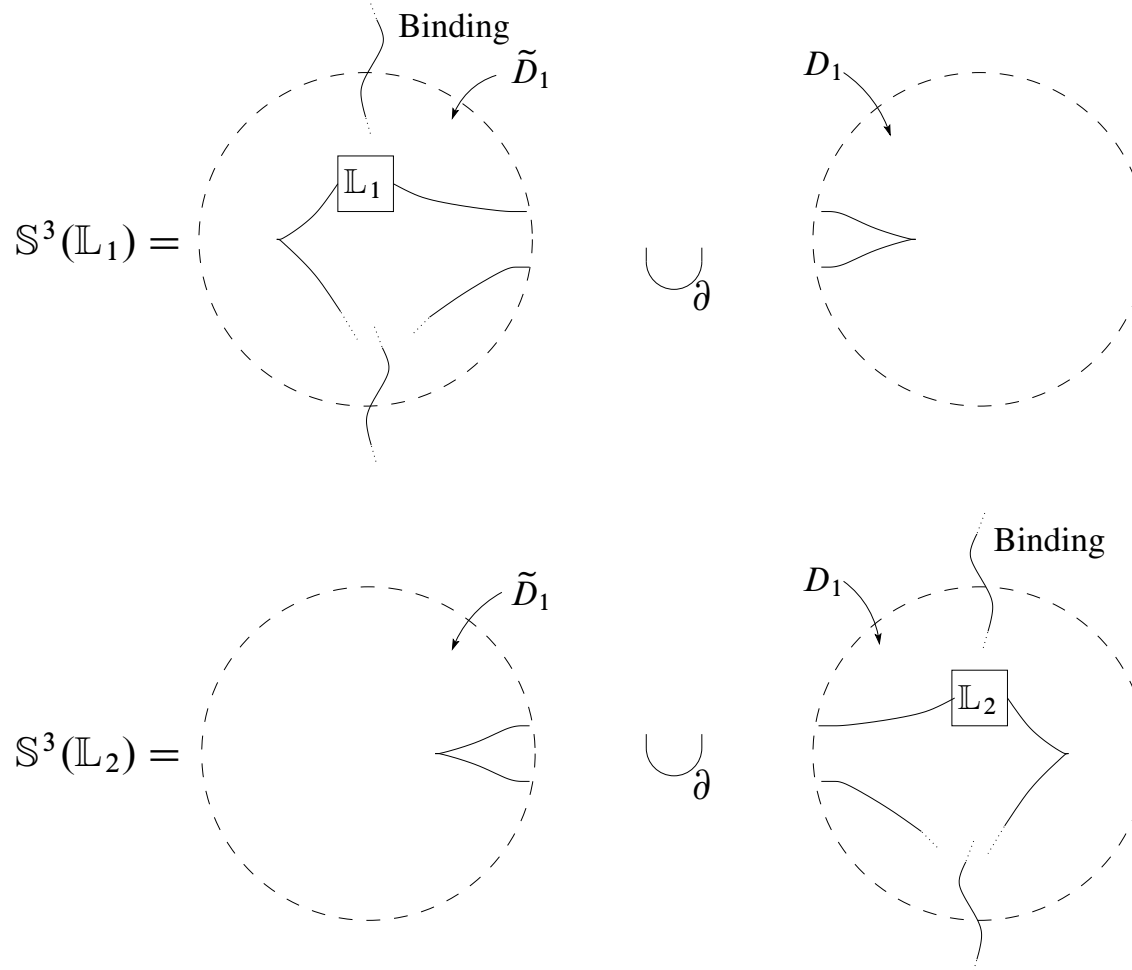

Figure 24: Our specific arrangement for performing the connected sum

the associated surgery link $\mathbb{L}_{2}$ to sit inside $D_{1}$ and $\widetilde{D}_{1}$ to be the ball in which $L_{2}$ comes close to $B_{2}$ (see the bottom row of Figure 24). We can form the connected sum

$$
\mathbb{S}^{3}\left(\mathbb{L}_{1} \sqcup \mathbb{L}_{2}\right)=\mathbb{S}^{3}\left(\mathbb{L}_{1}\right) \backslash D_{1} \cup_{\partial} \mathbb{S}^{2} \times[0,1] \cup_{\partial} \mathbb{S}^{3}\left(\mathbb{L}_{2}\right) \backslash \widetilde{D}_{1}
$$

where the gluing is determined by the naturally given embeddings (see Geiges [10, Section 4.12])

$$
\iota_{1}: D_{1} \hookrightarrow \mathbb{S}^{3} \text { and } \iota_{2}: \widetilde{D}_{1} \hookrightarrow \mathbb{S}^{3} .
$$

For a detailed discussion of connected sums of contact manifolds we point the reader to [10]. The induced contact structure is the connected sum $\xi_{\mathbb{L}_{1}} \# \xi_{\mathbb{L}_{2}}=\xi_{\mathbb{L}_{1} \sqcup \mathbb{L}_{2}}$ (see [10, Section 4.12]). The knots $L_{1}$ and $L_{2}$ are contained in this connected sum and, here, we can perform the Legendrian band sum as defined at the beginning of this section; we can perform a band sum which looks like given in Figure 25. Recall that we introduced a connected sum operation such that the open books $\left(P_{i}, \phi_{i}\right)$ glue together to give the open book $(P, \phi)$ where $P=P_{1} \cup_{h_{1}} P_{2}$ and $\phi$ is given as the composition of the two monodromies $\phi_{1}$ and $\phi_{2}$. To perform the connected sum operation such that the 


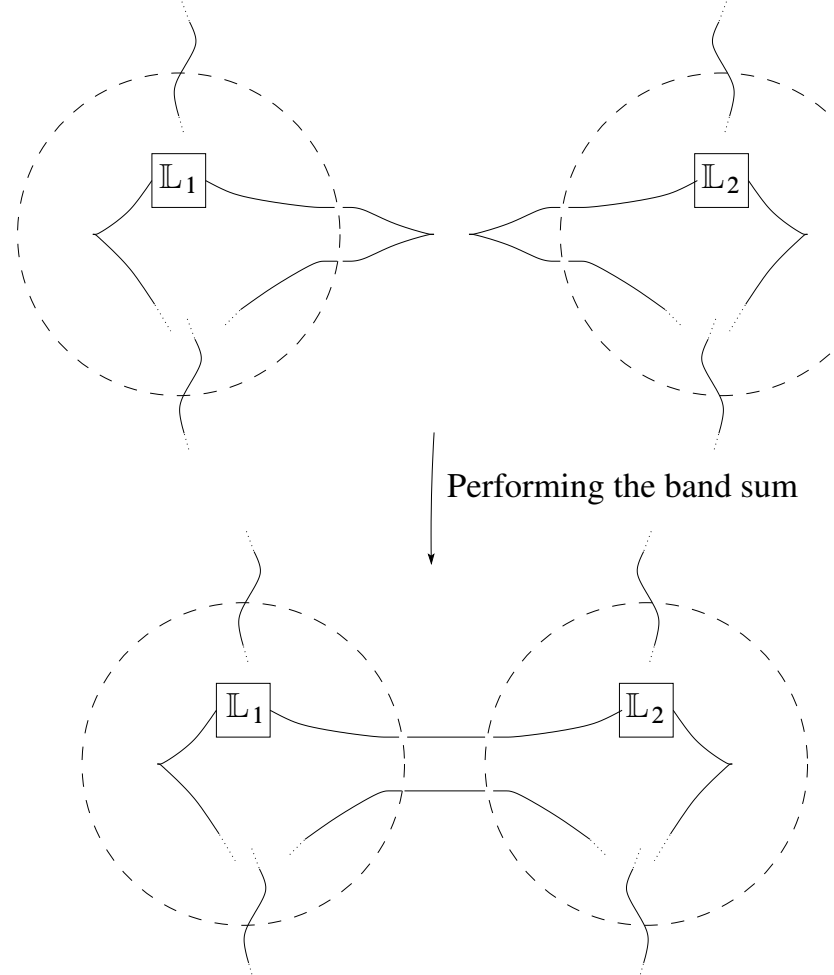

Figure 25: Performing a band sum of $L_{1}$ and $L_{2}$ inside $\mathbb{S}^{3}\left(\mathbb{L}_{1} \sqcup \mathbb{L}_{2}\right)$

open book structures are preserved, we have to modify the construction slightly. We modify the inclusion $\iota_{1}$ by composing it with a rotation about the $y$-axis with angle $\pi$. Without loss of generality we can think $L_{1} \cap \partial D_{1}$ and $L_{2} \cap \partial \widetilde{D}_{1}$ to be identified by the gluing induced by the inclusion maps $\iota_{1}$ and $\iota_{2}$. We can also assume that the rotation $r$ swaps the two intersection points $L_{1} \cap \partial D_{1}$. We obtain a new gluing map, $f$ say, and get

$$
Y=\mathbb{S}^{3}\left(\mathbb{L}_{1}\right) \backslash D_{1} \cup_{f} \mathbb{S}^{3}\left(\mathbb{L}_{2}\right) \backslash \widetilde{D}_{1}
$$

with induced contact structure $\xi$. With this identification the knots $L_{1}$ and $L_{2}$ glue together to give a knot $L$. This knot $L$ corresponds to a band sum of $L_{1}$ and $L_{2}$ on the page $P$ (after possibly applying Lemma 6.9). Recall that contact structures on $\mathbb{S}^{2} \times[0,1]$ are uniquely determined, up to isotopy, by the characteristic foliations on $\mathbb{S}^{2} \times\{j\}, j=0,1$ (see [10, Lemma 4.12.1, Theorem 4.9.4]). Consider the connected sum tube used in (6-6), and extend it with small collar neighborhoods of the boundaries of $\mathbb{S}^{3}\left(\mathbb{L}_{1}\right) \backslash D_{1}$ and $\mathbb{S}^{3}\left(\mathbb{L}_{2}\right) \backslash \widetilde{D}_{1}$. The characteristic foliation $\xi_{\mathbb{L}_{1}} \sqcup \mathbb{L}_{2}$ induces at the boundary will coincide with the characteristic foliation $\xi$ induces on a suitably chosen 
tubular neighborhood of $\partial D_{1} \cong \mathbb{S}^{2} \times[0,1]$ in $Y$. Thus, there is a contactomorphism between $v D_{1}$ and this thickened connected sum tube. Moreover, the contactomorphism can be extended to a contactomorphism

$$
\phi_{c}:(Y, \xi) \longrightarrow\left(\mathbb{S}^{3}\left(\mathbb{L}_{1} \sqcup \mathbb{L}_{2}\right), \xi_{\left.\mathbb{L}_{1} \sqcup \mathbb{L}_{2}\right)}\right.
$$

which just affects the connected sum tube and fixes the rest. As one can derive with some effort, this contactomorphism basically rotates the $\mathbb{S}^{2}$-factor once while going through the handle $\mathbb{S}^{2} \times[0,1]$. Thus, $\phi_{c}(L)$ looks like a band sum $L_{1} \#_{L b} L_{2}$ in $\mathbb{S}^{3}\left(\mathbb{L}_{1} \sqcup \mathbb{L}_{2}\right)$ after twisting the band once. Figure 26 applies.

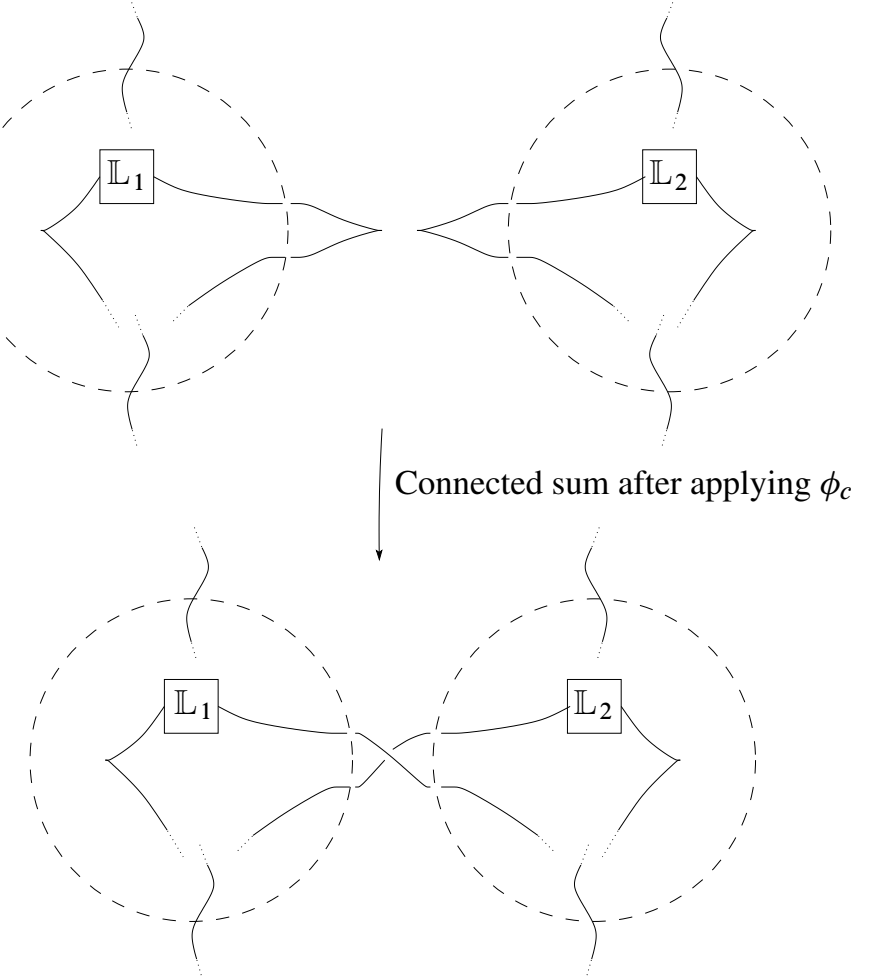

Figure 26: Schematic picture of the band bum after identifying $(Y, \xi)$ with $\left(\mathbb{S}^{3}\left(\mathbb{L}_{1} \sqcup \mathbb{L}_{2}\right), \xi_{\mathbb{L}_{1} \sqcup \mathbb{L}_{2}}\right)$

The following statement is due to Etnyre. Since there is no proof in the literature, we include a proof here for the convenience of the reader.

Proposition 6.11 (Etnyre [9]) Let $(Y, \xi, L)$ be a contact manifold with Legendrian knot and $(P, \phi)$ and open book adapted to $\xi$ with $L$ on its page such that the page 
framing and contact framing coincide. By stabilizing the open book once we can arrange either the stabilized knot $S_{+}(L)$ or $S_{-}(L)$ to sit on the page of the stabilized open book as indicated in Figure 27.
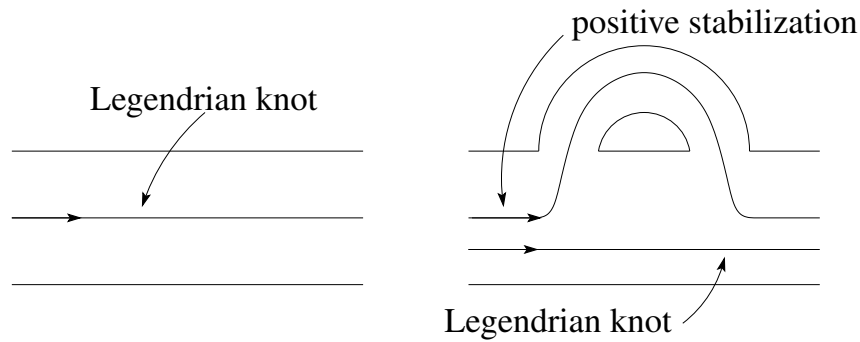

Figure 27: The stabilized open book and a positive Legendrian stabilization

The following result concerning the vanishing of the Legendrian invariant under positive stabilizations is due to Lisca, Ozsváth, Stipsicz and Szabó and follows from their connected sum formula given in [15]. Their proof carries over verbatim even for knots which are homologically non-trivial. Here we reprove a special case of [15, Theorem 7.2] using different methods.

Proposition 6.12 (Lisca-Ozsváth-Stipsicz-Szabó [15, Theorem 7.2]) Given any Legendrian knot $L$ in a contact manifold $(Y, \xi)$, we have $\widehat{\mathcal{L}}\left(S_{+}(L)\right)=0$.

Proof Let $(P, \phi)$ be an open book decomposition adapted to $(Y, \xi, L)$. By Proposition 6.11 we know that a stabilized open book $\left(P^{\prime}, \phi^{\prime}\right)$ carries the stabilized knot $S_{+}(L)$. Furthermore, from Figures 27 and 33 we can see how the induced Heegaard diagram (adapted to capturing the contact geometric information) will look like near the base point $w$. This is done in Figure 28. We may use Proposition 6.5 to check that the positioning of the point $w$ in Figure 28 is correct. First observe that $\widehat{\mathcal{L}}\left(S_{+}(L)\right)$ is the homology class induced by the point

$$
\left\{x_{1}, x_{2}, x_{3}, \ldots, x_{2 g}\right\} \text {. }
$$

Recall that by definition of the points $x_{i}$ every holomorphic disc emanating from $x_{i}$ is constant. Thus, a holomorphic disc emanating from $Q:=\left\{p, q, x_{3}, \ldots, x_{2 g}\right\}$ can only be non-constant at $p, q$. By orientation reasons and the placement of $w$ the shaded region is the only region starting at $p, q$ which can carry a holomorphic disc. Since it is disc-shaped, it does carry a holomorphic disc. Hence

$$
\widehat{\partial}^{w} Q=\left\{x_{1}, x_{2}, x_{3}, \ldots, x_{2 g}\right\}
$$

showing that $\widehat{\mathcal{L}}\left(S_{+}(L)\right)$ vanishes. 


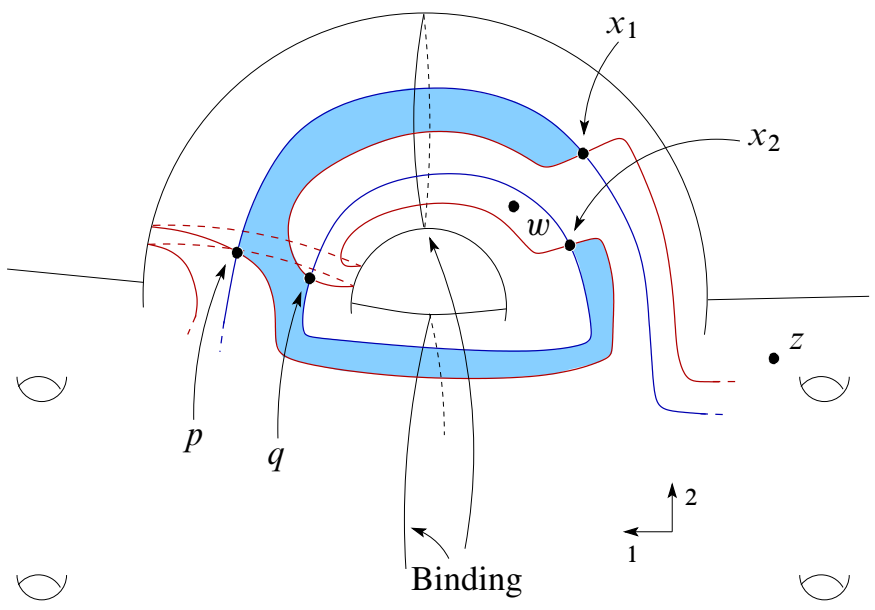

Figure 28: Parts of the Heegaard diagram induced by the open book carrying the stabilized knot

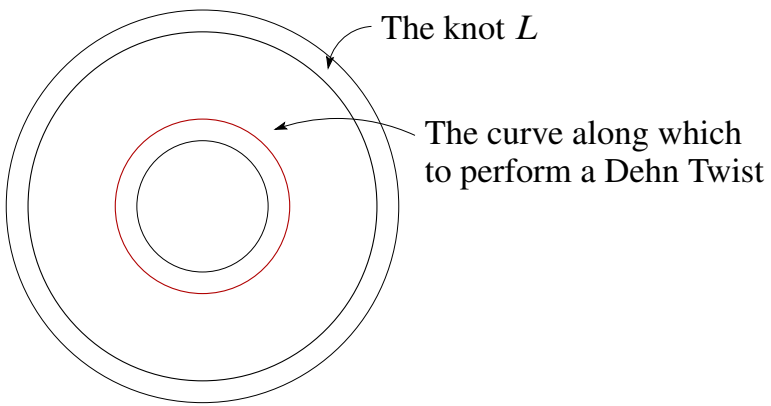

Figure 29: The open book necessary to carry the Legendrian unknot with $\mathrm{tb}=-1$ and $\operatorname{rot}=0$

Proof of Proposition 6.11 Given a triple $(Y, \xi, L)$, there is an open book $(P, \phi)$ adapted to $\xi$ such that $L$ sits on a page of the open book. By Proposition 6.6, Lemma 6.7 and Corollary 6.10 we perform a connected sum $(Y, \xi) \#\left(\mathbb{S}^{3}, \xi_{s t d}\right)$ on the level of open books using the open book of $\left(\mathbb{S}^{3}, \xi_{s t d}\right)$ pictured in Figure 29. By construction, the new open book carries the Legendrian knot $L_{2}$ pictured in Figure 30. In Figure 31 an isotopy is given, showing that $L_{2}$ corresponds to the band sum $L \#_{L b} L_{0}$ and, thus, represents $S_{ \pm}(L)$.

By Figure 29 what happens on the level of open books can be pictured as in Figure 32.

Proof of Proposition 6.5 Using Proposition 6.11, we have a tool to compare the open book orientation before and after the stabilization. We start with an open book adapted 


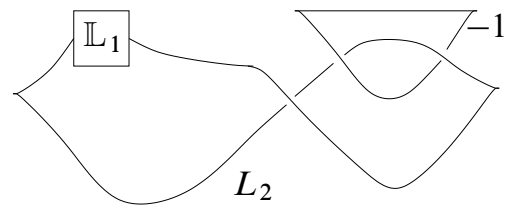

Figure 30: The knot $L_{2}$ in $(Y, \xi) \#\left(\mathbb{S}^{3}, \xi_{s t d}\right)$
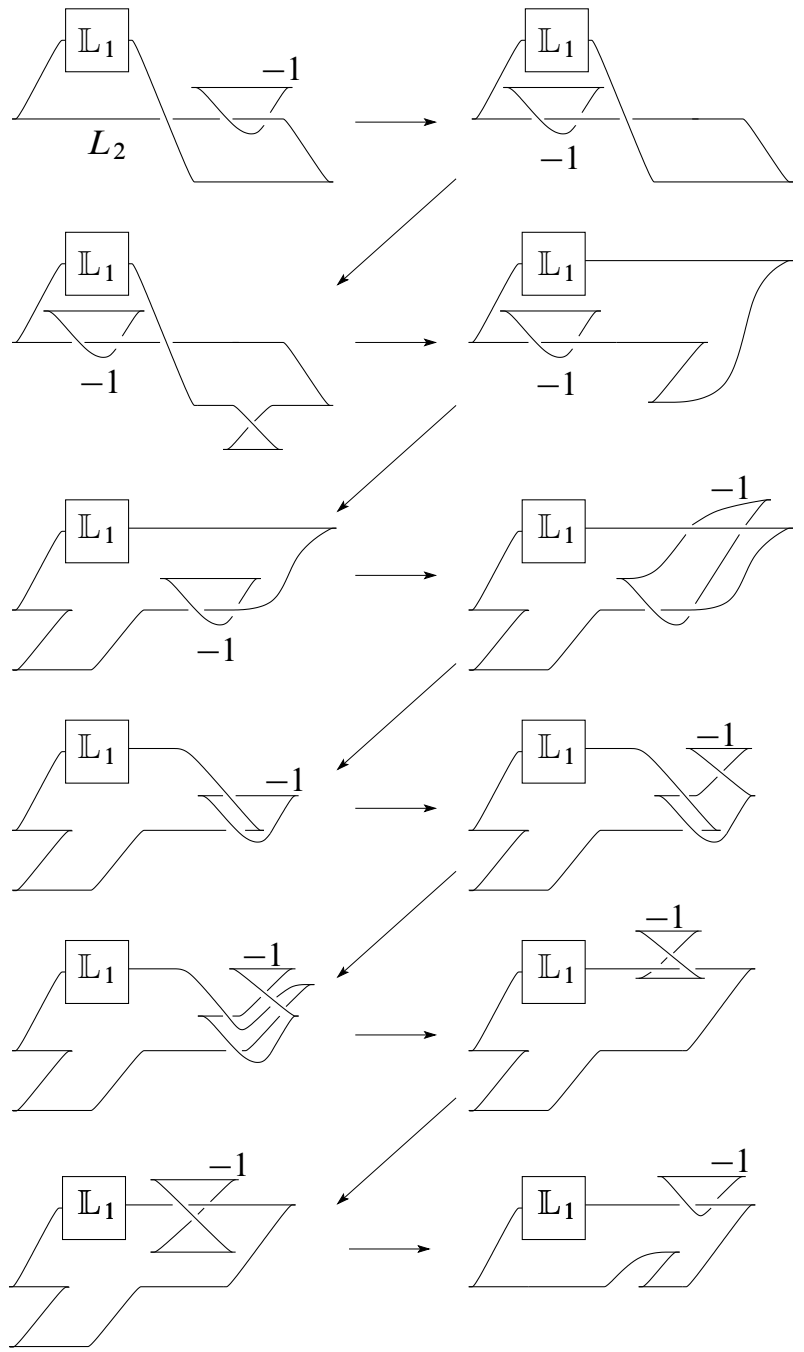

Figure 31: Legendrian isotopy showing that $L_{2}$ corresponds to the Legendrian band sum of $L$ with the Legendrian shark $L_{0}$ 

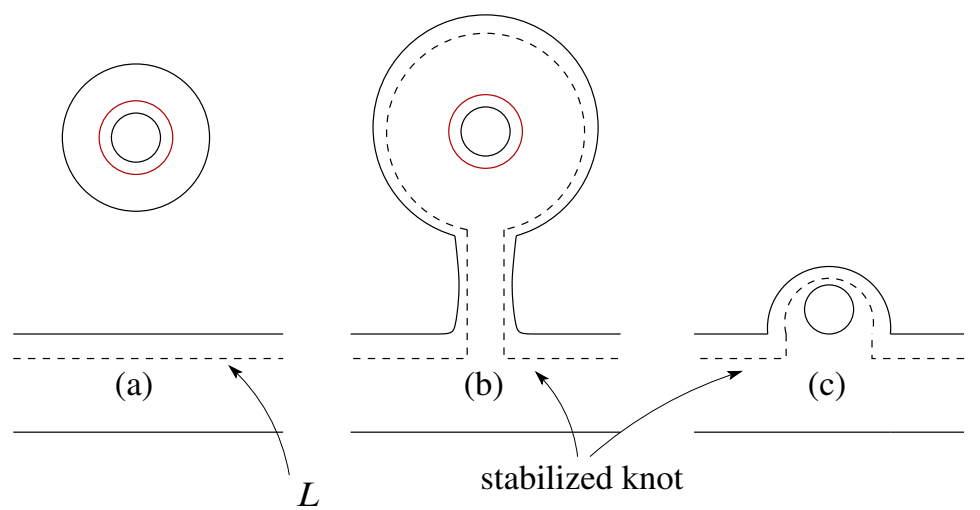

Figure 32: What happens during stabilization

to the triple $(Y, \xi, L)$ and choose an $L$-adapted cut system. By Proposition 6.11 we can generate an open book adapted to the positive stabilization by stabilizing the open book. Doing this appropriately, we may extend the cut system to an adapted cut system of the stabilized open book as indicated in Figure 33. Recall the rule with which the knot orientation is determined by the points $(w, z)$ (see remark in Section 2.4.2). In Figure 33 we can now compare the open book orientation of the stabilized knot with the orientation induced by the stabilization. We see that the orientations coincide. $\square$

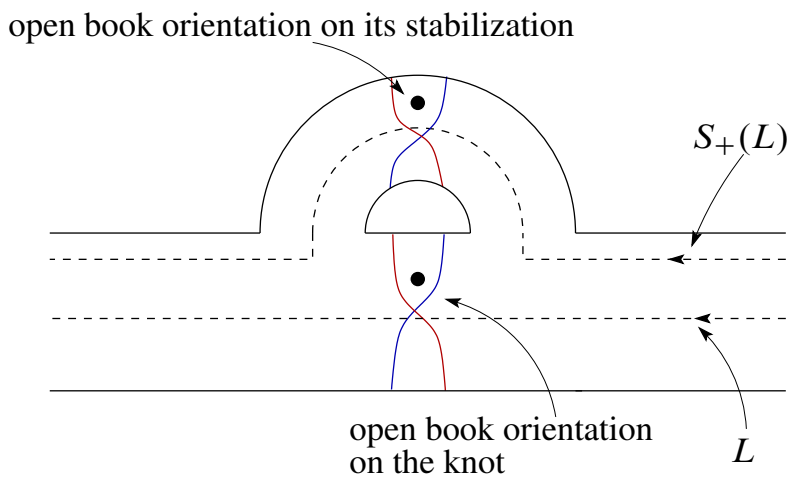

Figure 33: Comparing induced with open book orientation

\section{Applications - vanishing results of the contact element}

In this section we want to derive some applications of the theory developed in Section 4, Section 5 and Section 6. First to mention would be Proposition 7.1, which can 
also be derived using methods developed in [17]. There, Lisca and Stipsicz show that $(+1)$-contact surgery along stabilized Legendrian knots yield overtwisted contact manifolds, which implies the vanishing of the contact element. A second application would be Proposition 7.3, which is meant as a demonstration that calculating the Legendrian knot invariant and using Theorem 6.1 to get information about a contact element under investigation can be more convenient than using other methods, since the knot Floer homologies have additional structures we may use. A third application would be Theorem 7.4 which is a vanishing result of the contact element which can be easily read off from a surgery representation. This application uses the knot Floer homology for arbitrary knots and makes use of a phenomenon that seems to be special about these, namely that there are knots for which the knot Floer homology vanishes. We do not know any other example with this property.

Proposition 7.1 If $(Y, \xi)$ is obtained from $\left(Y^{\prime}, \xi^{\prime}\right)$ by $(+1)$-contact surgery along a Legendrian knot $L$ which can be destabilized, the element $c(\xi)$ vanishes.

Proof There are two cases to cover. Give the knot $L$ an orientation $o$. Suppose that

$$
(L, o)=S_{+}\left(L^{\prime}, o^{\prime}\right) .
$$

Then Proposition 6.12 shows the vanishing of $\widehat{\mathcal{L}}(L, o)$. By Theorem 6.1 the element $c(\xi)$ vanishes, too. Now assume that

$$
(L, o)=S_{-}\left(L^{\prime}, o^{\prime}\right)
$$

We see that

$$
(L, \bar{o})=\overline{S_{-}\left(L^{\prime}, o^{\prime}\right)}=S_{+}\left(L^{\prime}, \bar{o}\right),
$$

hence, $\widehat{\mathcal{L}}(L, \bar{o})=0$. By Theorem 6.1 again $c(\xi)=0$.

There are some immediate consequences we may derive from this theorem. The first corollary is well-known but with help of our results we are able to reprove it.

Corollary 7.2 (Ozsváth and Szabó) If $(Y, \xi)$ is overtwisted, the contact element vanishes.

Proof Recall that the surgery diagram given in Figure 34 is an overtwisted contact structure $\xi^{\prime}$ on $\mathbb{S}^{3}$.

This overtwisted contact structure is homotopic to $\xi_{\text {std }}$ as 2-plane fields (see Ding and Geiges [4]). By Eliashberg's classification theorem (see [7]), a connected sum of $(Y, \xi)$ with $\left(\mathbb{S}^{3}, \xi^{\prime}\right)$ does not change the contact manifold, that is,

$$
(Y, \xi)=(Y, \xi) \#\left(\mathbb{S}^{3}, \xi^{\prime}\right) \text {. }
$$



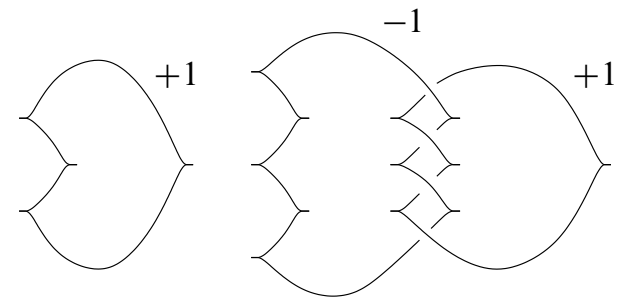

Figure 34: Surgery diagram for an overtwisted $\mathbb{S}^{3}$ in the homotopy class of $\xi_{\text {std }}$

Denote by $K$ the shark on the left of Figure 34. The manifold $(Y, \xi)$ admits a surgery representation $\mathbb{S}^{3}(\mathbb{L})$ where $\mathbb{L}=K \sqcup \mathbb{L}^{\prime}$. Furthermore, $K$ and $\mathbb{L}^{\prime}$ are not linked. Denote by $\left(Y^{\prime}, \xi^{\prime \prime}\right)$ the contact manifold with surgery representation $\mathbb{S}^{3}\left(\mathbb{L}^{\prime}\right)$. We obtain $(Y, \xi)$ out of $\left(Y^{\prime}, \xi^{\prime \prime}\right)$ by $(+1)$-contact surgery along $K$, which can be destabilized inside $Y^{\prime}$. Proposition 7.1 implies the vanishing of $c(\xi)$.

Remark 4 For a detailed discussion of the homotopy invariants of overtwisted contact structures on $\mathbb{S}^{3}$ see Ding, Geiges and Stipsicz [5].

Another consequence is that performing a simple Lutz twist along a transverse knot kills the contact element. The resulting contact structure is clearly overtwisted. Thus, by work of Ozsváth and Szabó the contact element vanishes. But besides this approach we can show the vanishing of the contact element without referring to overtwistedness at all. In [6], Ding, Geiges and Stipsicz present a surgical description for simple Lutz twists along transverse knots. This description involves $(+1)$-contact surgeries along a Legendrian approximation $L$ of the transverse knot and another Legendrian knot which is a stabilized version of $L$. Proposition 7.1 then implies the vanishing of the contact element.

When looking at a homologically trivial knot $L$, to show the vanishing of a contact element after surgery along $L$ it can be convenient to show the vanishing of $\widehat{\mathcal{L}}(L)$ and then apply Theorem 6.1, because of the various gradings on the knot Floer homological level. The following proposition is meant as an illustration of this fact.

Proposition 7.3 A (+1)-contact surgery along the Legendrian realizations $L_{n}$ given in Figure 35 of the Eliashberg-Chekanov twist knots $E_{n}$ with $n \in-2 \mathbb{N}$ all give contact manifolds with vanishing contact element.

Proof Since the invariant $\widehat{\mathcal{L}}\left(L_{n}\right)$ of the Legendrian realizations $L_{n}$ of the knots $E_{n}$ live in $\widehat{\operatorname{HFK}}\left(-\mathbb{S}^{3}, E_{n}\right)$, and because of the correspondence

$$
\widehat{\operatorname{HFK}}\left(-\mathbb{S}^{3}, E_{n}\right)=\widehat{\operatorname{HFK}}\left(\mathbb{S}^{3}, \bar{E}_{n}\right),
$$



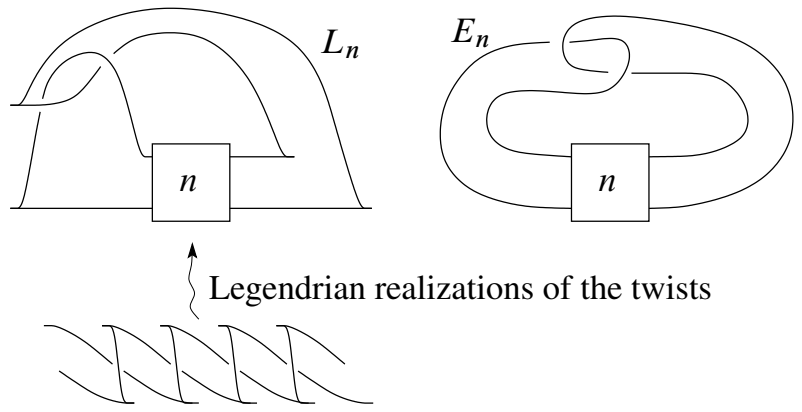

Figure 35: The Eliashberg-Chekanov twist knots $E_{n}$ and Legendrian realizations $L_{n}$

where $\bar{E}_{n}$ denotes the mirror knot, we have to compute the groups $\widehat{\mathrm{HFK}}\left(\mathbb{S}^{3}, \bar{E}_{n}\right)$. The knots are all alternating. Therefore we will stick to [20, Theorem 1.3] for a convenient computation of the groups. We compute the Alexander-Conway polynomial using its skein relation and get

$$
\Delta_{\bar{E}_{n}}(T)=(1-n)+\frac{n}{2}\left(T^{1}+T^{-1}\right) .
$$

To compute the signature of the knots $E_{n}$, we use the formula given in [20, Theorem 6.1] and see that all these knots have signature $\sigma\left(\bar{E}_{n}\right)=-n-2$. By [20, Theorem 1.3], which describes the knot Floer homology groups of an alternating knot in terms of the coefficients of the associated Alexander-Conway polynomial, the knot Floer homology of $\bar{E}_{n}$ looks like

$$
\widehat{\mathrm{HFK}}_{j}\left(\mathbb{S}^{3}, \bar{E}_{n}, i\right)= \begin{cases}\mathbb{Z}^{-n / 2} & \text { if } i=-1, j=-1+\frac{-n-2}{2}, \\ \mathbb{Z}^{|1-n|} & \text { if } i=0, j=\frac{-n-2}{2}, \\ \mathbb{Z}^{-n / 2} & \text { if } i=1, j=1+\frac{-n-2}{2}, \\ 0 & \text { otherwise. }\end{cases}
$$

According to Ozsváth and Stipsicz [19], the Legendrian invariant $\widehat{\mathcal{L}}\left(L_{n}\right)$ lives in $\widehat{\mathrm{HFK}}_{M\left(L_{n}\right)}\left(-\mathbb{S}^{3}, E_{n}, A\left(L_{n}\right)\right)$ where $A\left(L_{n}\right)$ is the Alexander grading of $L_{n}$ and $M\left(L_{n}\right)$ is called Maslov grading. These gradings are computed using the formulas (see [19])

$$
\begin{aligned}
2 \cdot A\left(L_{n}\right) & =\operatorname{tb}\left(L_{n}\right)-\operatorname{rot}\left(L_{n}\right)+1 \\
d_{3}\left(\xi_{s t d}\right) & =2 A\left(L_{n}\right)-M\left(L_{n}\right),
\end{aligned}
$$

where $d_{3}$ denotes the Hopf-invariant (see Gompf and Stipsicz [11]). However, note that with the conventions used in Heegaard Floer theory $d_{3}\left(\xi_{s t d}\right)=0$. With a straightforward computation we see that $\operatorname{tb}\left(L_{n}\right)=-4$ and $\operatorname{rot}\left(L_{n}\right)=1$, which give the following 
Alexander gradings and Maslov gradings

$$
\begin{gathered}
A\left(L_{n}\right)=-1 \\
M\left(L_{n}\right)=-2 .
\end{gathered}
$$

Consequently, we can show, by using the computed Alexander and Maslov gradings, that for every knot $L_{n}, n \neq 0$, the invariant $\widehat{\mathcal{L}}\left(L_{n}\right)$ is an element of a vanishing subgroup of $\widehat{\operatorname{HFK}}\left(\mathbb{S}^{3}, \bar{E}_{n}\right)$. To show the vanishing of $\widehat{\mathcal{L}}\left(L_{0}\right)$ we observe that $L_{0}$ can be destabilized.

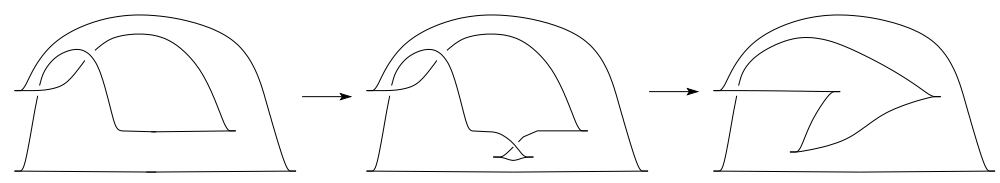

Figure 36: The Legendrian isotopy showing that $L_{0}$ can be destabilized

The isotopy is pictured in Figure 36. By Proposition $7.1 c\left(\xi_{L_{0}}^{+}\right)$vanishes, too. Using Theorem 6.1 the proposition follows.

The following theorem is a new vanishing result of the contact element, which uses the knot Floer homology for arbitrary knots. Furthermore, we make use of the fact that in $\mathbb{S}^{2} \times \mathbb{S}^{1}$ there are homologically non-trivial knots whose associated knot Floer homology vanishes.

Theorem 7.4 Let $(Y, \xi)$ be a contact manifold given as a contact surgery along a Legendrian link in $\left(\mathbb{S}^{3}, \xi_{\text {std }}\right)$. If the surgery diagram contains a configuration like given in Figure 37, the contact element $c(Y, \xi)$ vanishes.

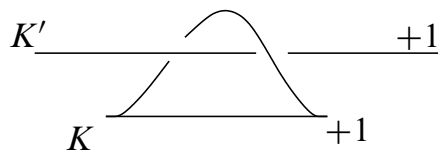

Figure 37: Configuration in a surgery diagram of $(Y, \xi)$ killing the contact element

Proof We start looking at the knot Floer homology group of the pair $\left(\mathbb{S}^{2} \times \mathbb{S}^{1}, G\right)$ where $G$ is a specific knot representing a generator of $H_{1}\left(\mathbb{S}^{2} \times \mathbb{S}^{1}\right)$ :

Figure 38 is a Heegaard diagram adapted to this specific knot $G$. A straightforward calculation gives $\widehat{\mathrm{HFK}}\left(\mathbb{S}^{2} \times \mathbb{S}^{1}, G\right)=0$. In Figure 39 we see a surgery diagram of $\mathbb{S}^{2} \times \mathbb{S}^{1}$ with the knot $G$ in it. 


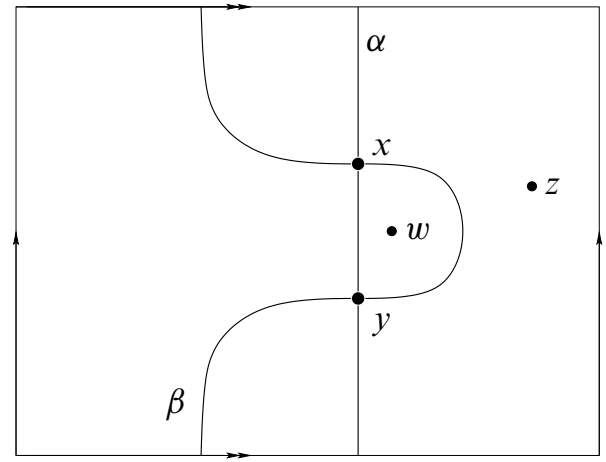

Figure 38: Heegaard diagram adapted to $G$

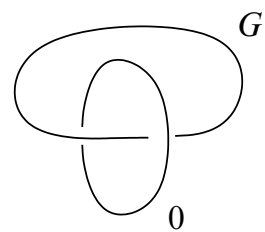

Figure 39: Surgery diagram of $\mathbb{S}^{2} \times \mathbb{S}^{1}$ with knot $G$ in it

Returning to Figure 37, we can interpret $K^{\prime}$ as an ordinary knot and remove it from the surgery description. We obtain a contact manifold $\left(Y^{\prime} \# \mathbb{S}^{2} \times \mathbb{S}^{1}, \xi^{\prime}\right)$ and $K^{\prime}$ is a Legendrian knot in it. A $(+1)$-contact surgery along $K^{\prime}$ will yield $(Y, \xi)$. Furthermore, as a topological knot, $K^{\prime}$ can be written as $K^{\prime \prime} \# G$ where $K^{\prime \prime} \subset Y$ and $G \subset \mathbb{S}^{2} \times \mathbb{S}^{1}$ is the knot given in Figure 39. Hence, we have (see Lisca, Ozsváth, Stipsicz and Szabó [15])

$$
\widehat{\mathrm{HFK}}\left(Y^{\prime} \#\left(\mathbb{S}^{2} \times \mathbb{S}^{1}\right), K^{\prime}\right)=\widehat{\operatorname{HFK}}\left(Y^{\prime}, K^{\prime \prime}\right) \otimes \widehat{\operatorname{HFK}}\left(\mathbb{S}^{2} \times \mathbb{S}^{1}, G\right)=0 .
$$

The same holds if we reverse the orientation on the manifold. We perform a $(+1)-$ contact surgery along $K^{\prime}$ to obtain $(Y, \xi)$. Denote by $W$ the induced cobordism. By Theorem 6.1 this induces a map

$$
\Gamma_{-W}: \widehat{\mathrm{HFK}}\left(-Y^{\prime} \#\left(\mathbb{S}^{2} \times \mathbb{S}^{1}\right), K^{\prime}\right) \longrightarrow \widehat{\mathrm{HF}}(-Y)
$$

with $c(Y, \xi)=\Gamma_{-W}\left(\widehat{\mathcal{L}}\left(K^{\prime}\right)\right)$. So, the contact element vanishes, since $\widehat{\mathcal{L}}\left(K^{\prime}\right)=0$.

\section{References}

[1] G E Bredon, Topology and geometry, Graduate Texts in Mathematics 139, Springer, New York (1993) MR1224675 
[2] F Ding, H Geiges, Symplectic fillability of tight contact structures on torus bundles, Algebr. Geom. Topol. 1 (2001) 153-172 MR1823497

[3] F Ding, H Geiges, A Legendrian surgery presentation of contact 3-manifolds, Math. Proc. Cambridge Philos. Soc. 136 (2004) 583-598 MR2055048

[4] F Ding, H Geiges, Handle moves in contact surgery diagrams, J. Topol. 2 (2009) 105-122 MR2499439

[5] F Ding, H Geiges, A I Stipsicz, Surgery diagrams for contact 3-manifolds, Turkish J. Math. 28 (2004) 41-74 MR2056760

[6] F Ding, H Geiges, A I Stipsicz, Lutz twist and contact surgery, Asian J. Math. 9 (2005) 57-64 MR2150691

[7] Y Eliashberg, Classification of overtwisted contact structures on 3-manifolds, Invent. Math. 98 (1989) 623-637 MR1022310

[8] Y Eliashberg, Topological characterization of Stein manifolds of dimension > 2, Internat. J. Math. 1 (1990) 29-46 MR1044658

[9] J B Etnyre, Lectures on open book decompositions and contact structures, from: "Floer homology, gauge theory, and low-dimensional topology", Clay Math. Proc. 5, Amer. Math. Soc., Providence, RI (2006) 103-141 MR2249250

[10] H Geiges, An introduction to contact topology, Cambridge Studies in Advanced Mathematics 109, Cambridge University Press, Cambridge (2008) MR2397738

[11] R E Gompf, A I Stipsicz, 4-manifolds and Kirby calculus, Graduate Studies in Mathematics 20, American Mathematical Society, Providence, RI (1999) MR1707327

[12] K Honda, W H Kazez, G Matić, The contact invariant in sutured Floer homology, Invent. Math. 176 (2009) 637-676 MR2501299

[13] K Honda, W H Kazez, G Matić, On the contact class in Heegaard Floer homology, J. Diff. Geom. (to appear)

[14] A Juhász, Holomorphic discs and sutured manifolds, Algebr. Geom. Topol. 6 (2006) 1429-1457 MR2253454

[15] P Lisca, P Ozsváth, A I Stipsicz, Z Szabó, Heegaard Floer invariants of Legendrian knots in contact three-manifolds, J. Eur. Math. Soc. (JEMS) 11 (2009) 1307-1363 MR2557137

[16] P Lisca, A I Stipsicz, Ozsváth-Szabó invariants and tight contact three-manifolds I, Geom. Topol. 8 (2004) 925-945 MR2087073

[17] P Lisca, A I Stipsicz, Notes on the contact Ozsváth-Szabó invariants, Pacific J. Math. 228 (2006) 277-295 MR2274521

[18] P Lisca, A I Stipsicz, Ozsváth-Szabó invariants and tight contact three-manifolds II, J. Differential Geom. 75 (2007) 109-141 MR2282726 
[19] P Ozsváth, A I Stipsicz, Contact surgeries and the transverse invariant in knot Floer homology arXiv:0803.1252

[20] P Ozsváth, Z Szabó, Heegaard Floer homology and alternating knots, Geom. Topol. 7 (2003) 225-254 MR1988285

[21] P Ozsváth, Z Szabó, Holomorphic disks and knot invariants, Adv. Math. 186 (2004) 58-116 MR2065507

[22] P Ozsváth, Z Szabó, Holomorphic disks and three-manifold invariants: properties and applications, Ann. of Math. (2) 159 (2004) 1159-1245 MR2113020

[23] P Ozsváth, Z Szabó, Holomorphic disks and topological invariants for closed threemanifolds, Ann. of Math. (2) 159 (2004) 1027-1158 MR2113019

[24] P Ozsváth, Z Szabó, Heegaard Floer homology and contact structures, Duke Math. J. 129 (2005) 39-61 MR2153455

[25] B Sahamie, Dehn Twists and Heegaard Floer Homology, $\mathrm{PhD}$ thesis, Universität zu Köln (2009)

[26] A I Stipsicz, V Vértesi, On invariants for Legendrian knots, Pacific J. Math. 239 (2009) 157-177 MR2449016

Mathematisches Institut der Universität zu Köln

Weyertal 86-90, 50931 Köln, Germany

Mathematisches Institut der LMU München

Theresienstrasse 39, 80333 München, Germany

sahamie@math. Imu.de

http://www.math.lmu.de/ sahamie

Received: 22 April $2009 \quad$ Revised: 5 November 2009 